\title{
Iridium-Catalyzed Asymmetric Allylic Substitution with Aryl Zinc Reagents
}

\author{
Alexandre Alexakis*, Samir El Hajjaji, Damien Polet, Xavier Rathgeb \\ Department of Organic Chemistry, University of Geneva, 30 quai Ernest-Ansermet, \\ Geneva 4, Switzerland $\mathrm{CH}-1211$ \\ Fax: +41 22/328.73.96; e-mail: alexandre.alexakis@chiorg.unige.ch
}

\section{Supporting Information}

\section{List of contents (pages)}

General procedures

Compound 2: Chiral-GC for 2, ${ }^{1} \mathrm{H}-\mathrm{NMR}$ spectra and ${ }^{13} \mathrm{C}-\mathrm{NMR}$

Compound 4: Chiral-SFC for 4, ${ }^{1} \mathrm{H}-\mathrm{NMR}$ spectra

Compound 6: Chiral-SFC for 6, ${ }^{1} \mathrm{H}-\mathrm{NMR}$ spectra and ${ }^{13} \mathrm{C}-\mathrm{NMR}$

\section{General:}

All reactions were conducted under inert atmosphere. All commercially available reagents were used without further purification unless otherwise noted. All solvent were dried on alumina columns and degassed before used. The evolution of reactions was monitored by GC-MS (EI mode). Flash column chromatography was performed with silica gel 32-63 $\mu \mathrm{m}$, $60 \AA .{ }^{1} \mathrm{H}(400 \mathrm{MHz})$ and ${ }^{13} \mathrm{C}(100 \mathrm{MHz}) \mathrm{NMR}$ spectra were recorded in $\mathrm{CDCl}_{3}$, and chemical shift $(\delta)$ are given in ppm relative to residual $\mathrm{CHCl}_{3}$. Optical rotations were measured at $20{ }^{\circ} \mathrm{C}$ 
in the stated solvent; $[\alpha]_{\mathrm{D}}$ values are given in $10^{-1} \mathrm{deg}^{2} \mathrm{gm}^{-1}$. Enantiomeric excesses were determined by chiral-GC (capillary column, $10 \mathrm{psi}_{2}$ ) or chiral-SFC (Supercritical Fluid Chromatography) with the stated column. Temperature programs are described as follows: initial temperature $\left({ }^{\circ} \mathrm{C}\right)$ - initial time ( $\left.\mathrm{min}\right)$ - temperature gradient $\left({ }^{\circ} \mathrm{C} / \mathrm{min}\right)$ - final temperature $\left({ }^{\circ} \mathrm{C}\right)$ - final time (min). Gradient programs are described as follows: initial methanol concentration (\%), initial time ( $\mathrm{min})$, percent gradient of methanol $(\% / \mathrm{min})$, final methanol concentration $(\%)$. Retention times $\left(R_{\mathrm{T}}\right)$ are given in min and corresponding peaks are checked by UV spectrum.

\section{General procedure (GP) for the Ir-catalyzed allylic alkylation:}

In a flame-dried Schlenk tube, chiral ligand $(4.4 \%),[\operatorname{Ir}(\mathrm{COD}) \mathrm{Cl}]_{2}(2 \%)$ were added in dry THF ( $1 \mathrm{ml}$ for each mmol of starting material). The resulting orange solution was stirred for 10-15 minutes at RT. Then substrate (1 eq.) was added into the Schlenk following by nucleophilic solution. The reaction mixture was stirred at RT overnight. The mixture was then hydrolyzed with water, few drops of $\mathrm{HCl} 10 \%$ and ether. The organic layer was separated and the aqueous layer was washed 3 times with ether. The combined organic layers were dried with anhydrous $\mathrm{MgSO}_{4}$, filtered and concentrated in vacuo.

\section{Preparation of nucleophilic solution 1 (NS1):}

In a flame-dried flask containing dry THF ( $2 \mathrm{ml}$ for each mmol of starting material), $\mathrm{ZnBr}_{2}$ ( 0.75 eq.), $\operatorname{LiBr}(1.5$ eq.) were added. The solution was stirred for 10-15 minutes at RT. Then Grignard reagent (1.5 eq.) was added into the flask and nucleophilic solution was ready to be used (equivalents were relative to substrate).

\section{Preparation of nucleophilic solution 2 (NS2):}

In a flame-dried flask containing dry THF (1.4 ml for each mmol of starting material), 2bromonaphthalene (1.5 eq.) was added. The solution was cooled to $-78^{\circ} \mathrm{C}$ before adding $t$ BuLi 1.5M (3 eq.). The bath is removed and the solution was stirred at RT. The reaction was followed by GC-MS. After complete conversion, $\mathrm{ZnBr}_{2}$ (0.75 eq.) was added into the flask at RT. Nucleophilic solution was ready to be used (equivalents were relative to substrate). 


\section{1-((S)-1-cyclohexylallyl)benzene 2:}

The reaction was done on $0.5 \mathrm{mmol}$ scale of starting material 1 following GP/NS1 and using ligand (S,S,S). The product was purified by flash column chromatography (silica, pentane), to afford the SN2'-SN2 mixture (61/49) as a colourless liquid (87.6

$\mathrm{mg}, 88 \%$ yield). Ee of $74 \%$ were mesured by chiral GC with a LIPODEX-E column (program: $60^{\circ}-0^{\prime}-1^{\circ}{ }^{\prime}-130^{\circ}-15^{\circ}{ }^{\prime}-170^{\circ}-5^{\prime}$, speed $49 \mathrm{~cm} / \mathrm{s}$ ), $\mathrm{R}_{\mathrm{T}}$ : $67.90(\mathrm{~S})$ and 68.59 (R). ${ }^{1} \mathrm{H}$ NMR (400 MHz, $\left.\mathrm{CDCl}_{3}\right)$ : 7.39-7.22 (m, 5H), 6.07 (dt, 1H, J = 17.4, 9.4 Hz), 5.12-5.07 (m, $\left.2 \mathrm{H}\right), 3.01(\mathrm{t}, 1 \mathrm{H}, \mathrm{J}=9.1$ $\mathrm{Hz}), 2.00-0.85$ (m, 11H). ${ }^{13} \mathrm{C} \mathrm{NMR}\left(100 \mathrm{MHz}, \mathrm{CDCl}_{3}\right)$ : 144.2, 141.3, 128.4 (2C), 128.0 (2C), 126.0, 114.9, 57.7, 42.2, 31.4, 31.4, 26.6, 26.5.
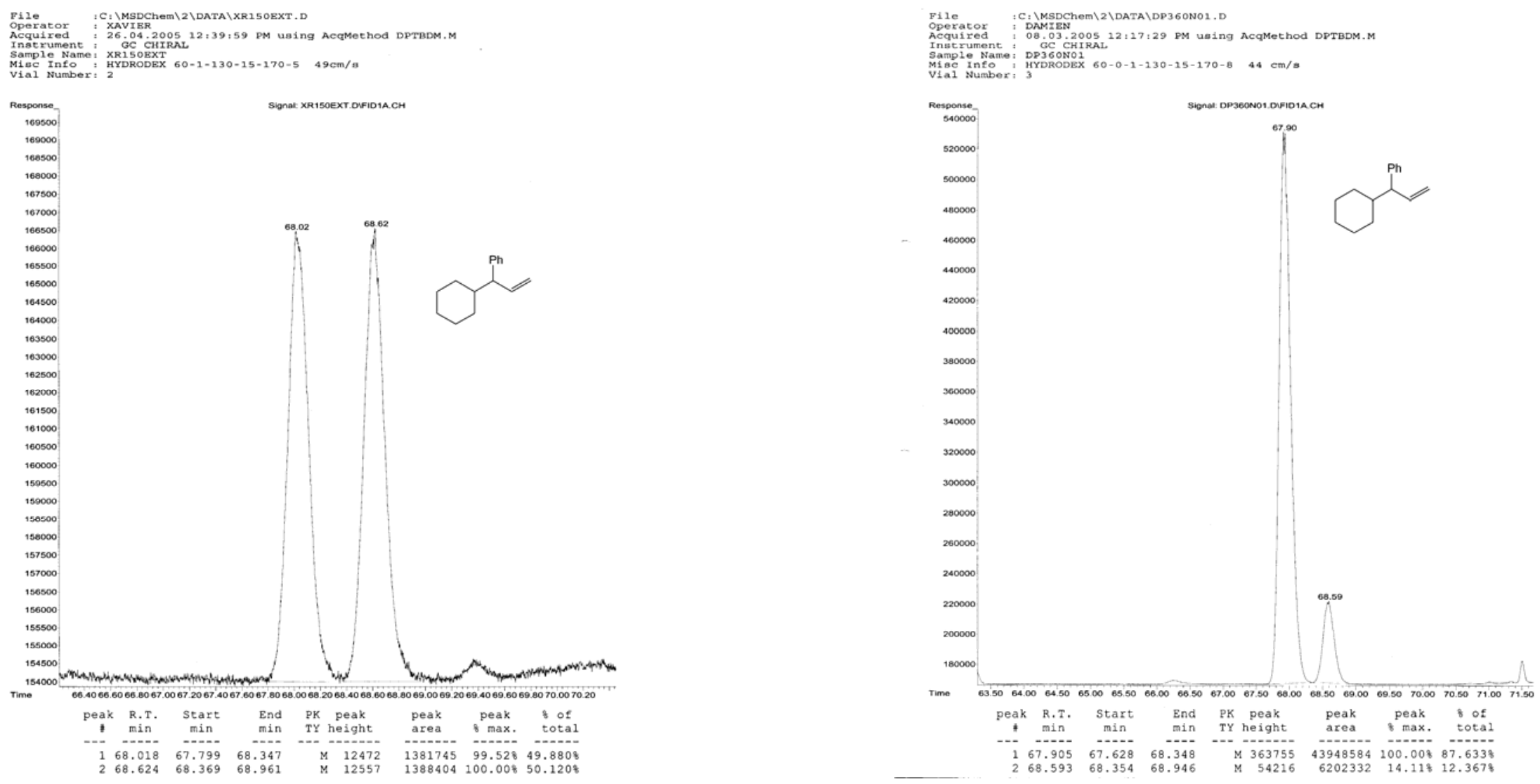
${ }^{1} \mathrm{H}-\mathrm{NMR}$

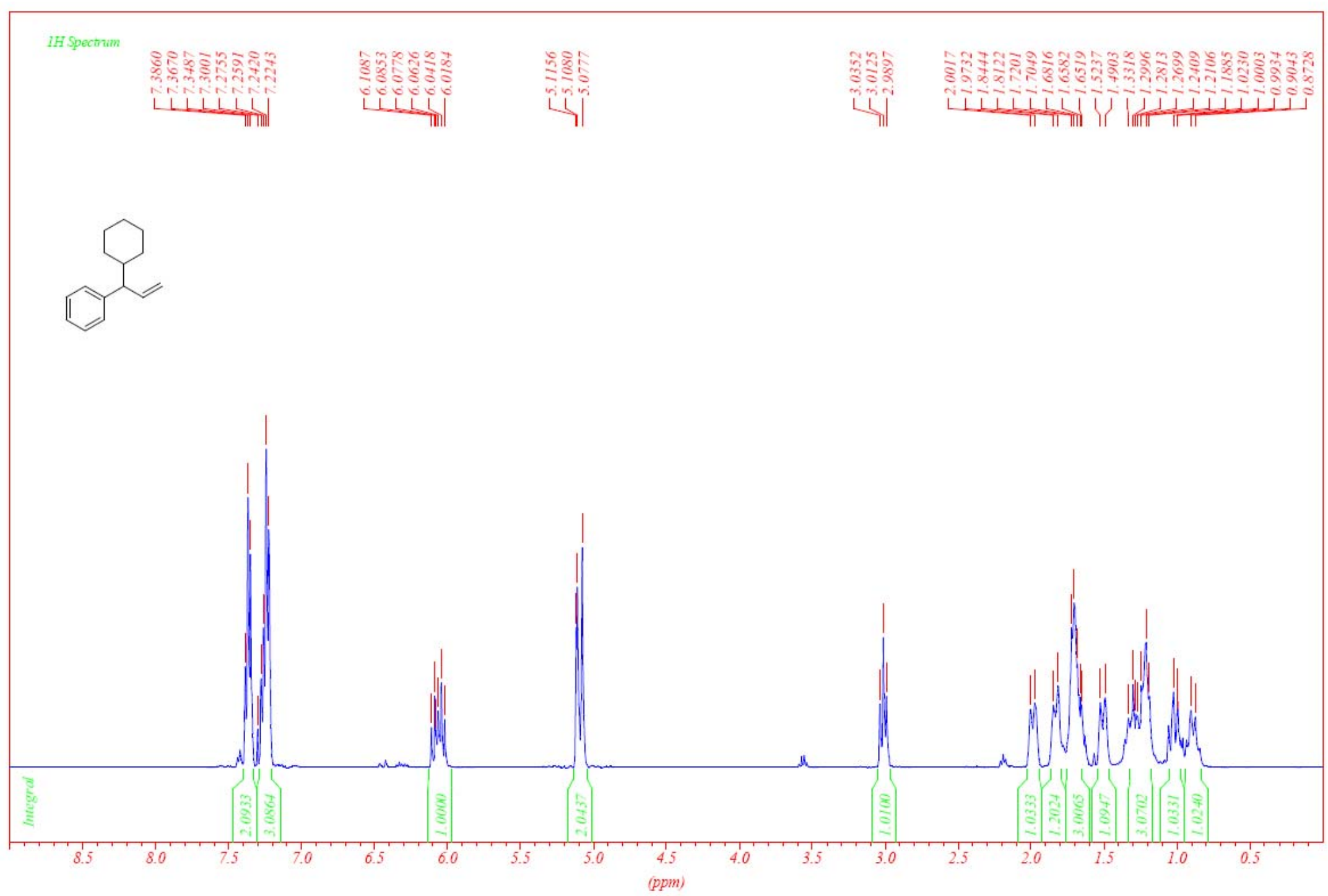


${ }^{13} \mathrm{C}-\mathrm{NMR}$

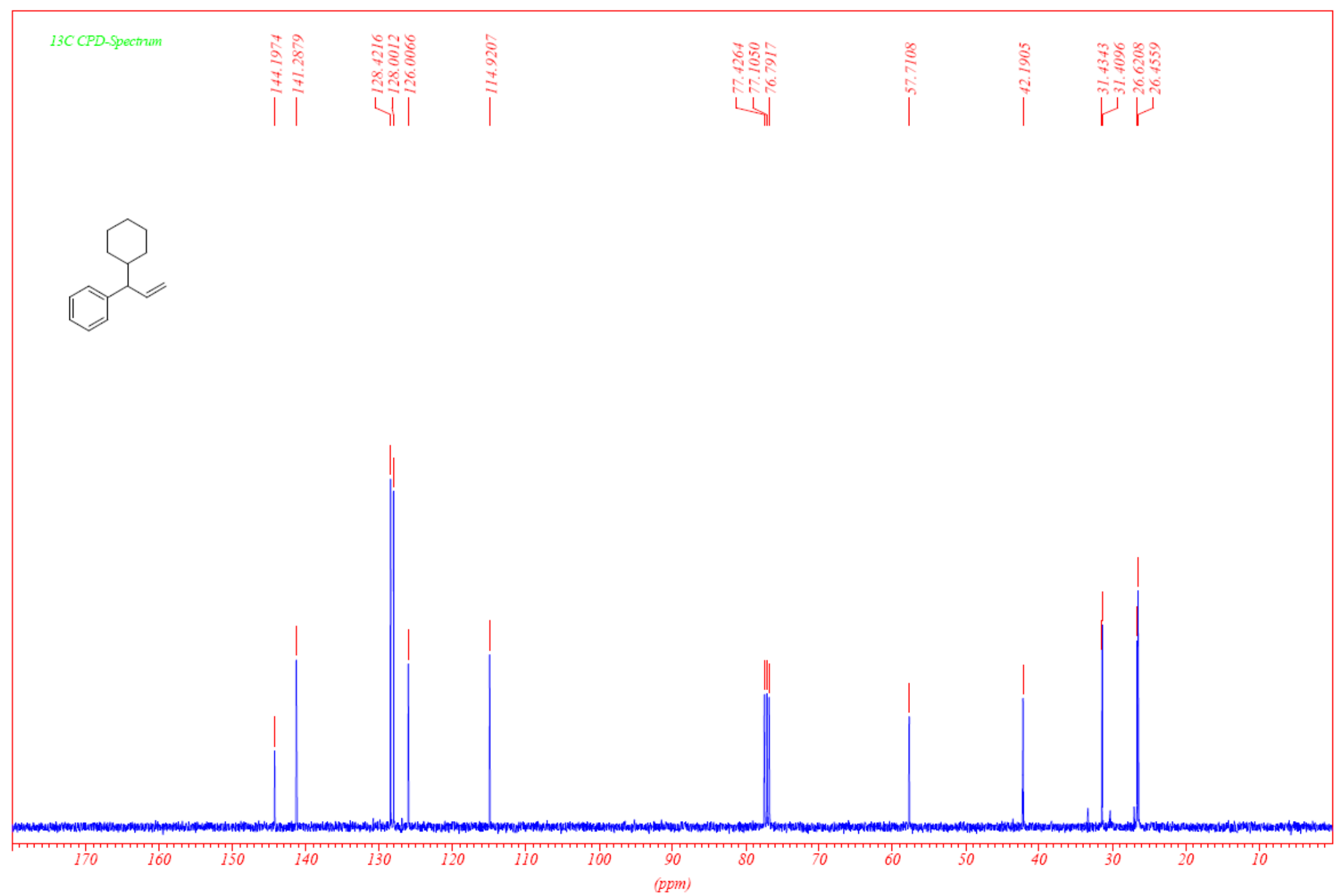




\section{1-(1-(2-methoxyphenyl)allyl)benzene 4:}

The reaction proceeds on $0.5 \mathrm{mmol}$ scale of starting material 3 following GP/NS1 and using ligand L1 (R,R,R). The product was

OMe purified by flash column chromatography (silica, pentane), to afford the SN2'-SN2 mixture (45/55) as a slightly yellow liquid (n.d. yield). Ee of 78\% was measured by chiral SFC with a OJ-H column (program: 1\% MeOH-4'-1-20\%, 200 bar, $2 \mathrm{ml} / \mathrm{min}, 30^{\circ} \mathrm{C}$ ). $\mathrm{R}_{\mathrm{T}}$ : 7.61 (R) and $8.26(\mathrm{~S}) .{ }^{1} \mathrm{H}$ NMR $\left(400 \mathrm{MHz}, \mathrm{CDCl}_{3}\right) \delta$ 6.80-7.70 (m, 9H), $6.37(\mathrm{~m}, 1 \mathrm{H}),, 5.23(\mathrm{~m}, 2 \mathrm{H}), 4.96(\mathrm{dt}, 1 \mathrm{H}, \mathrm{J}=17.1 \mathrm{~Hz}, \mathrm{~J}=1.5 \mathrm{~Hz}), 4.71(\mathrm{~d}, 1 \mathrm{H}, \mathrm{J}$

$=7.1 \mathrm{~Hz}), 3.79(\mathrm{~s}, 3 \mathrm{H})$.

\section{OJ 18-4-1-20 200 bars $2 \mathrm{ml} / \mathrm{min} \quad 30 \mathrm{C}$}

\begin{tabular}{|c|c|c|}
\hline $\begin{array}{l}\text { Acq. Method } \\
\text { Acq. Operator } \\
\text { Injection Date } \\
\text { Sample Name }\end{array}$ & $\begin{array}{l}\text { OJ1-4.M } \\
\text { Karine } \\
26-02-03 \quad 13: 29: 20 \\
\text { KC5-512 }\end{array}$ & $\begin{aligned} \text { Seq. Line } & - \\
\text { Vial } & 1001 \\
\text { Inj } & - \\
\text { Inj Volume } & : \text { Unknown }\end{aligned}$ \\
\hline $\begin{array}{l}\text { Analysis Method } \\
\text { CHIRALCEL OJ }\end{array}$ & $\begin{array}{l}\text { C: } \backslash \text { BERGER } 1 \backslash \text { METHODS } \backslash O J 1-4 . M \\
\text { (modified after loading) }\end{array}$ & \\
\hline
\end{tabular}

CHIRALCEL OU
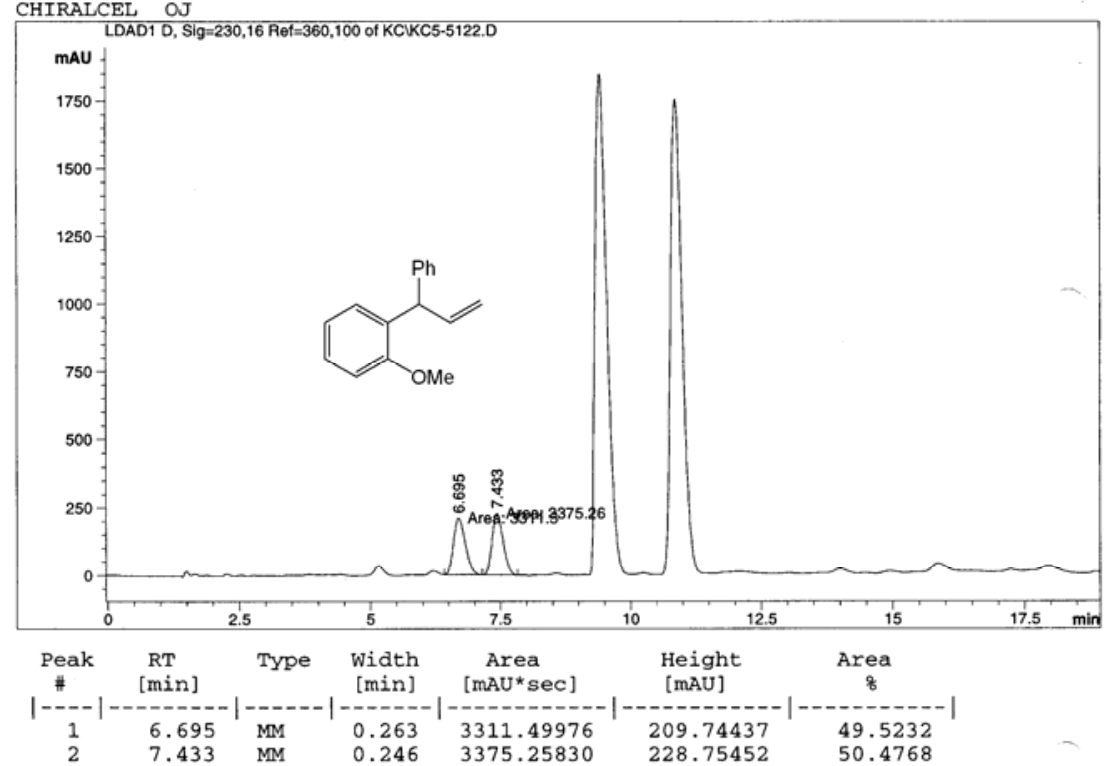

OJ-H $18-4-1-20 \% \quad 200$ bars $2 \mathrm{ml} / \mathrm{min} \quad 30 \mathrm{C}$

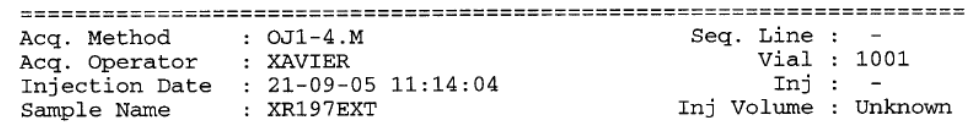

Sample Name
Analysis Method : C: $\backslash$ BERGER $\backslash 1 \backslash$ METHODS $\backslash$ OJ1-4.M

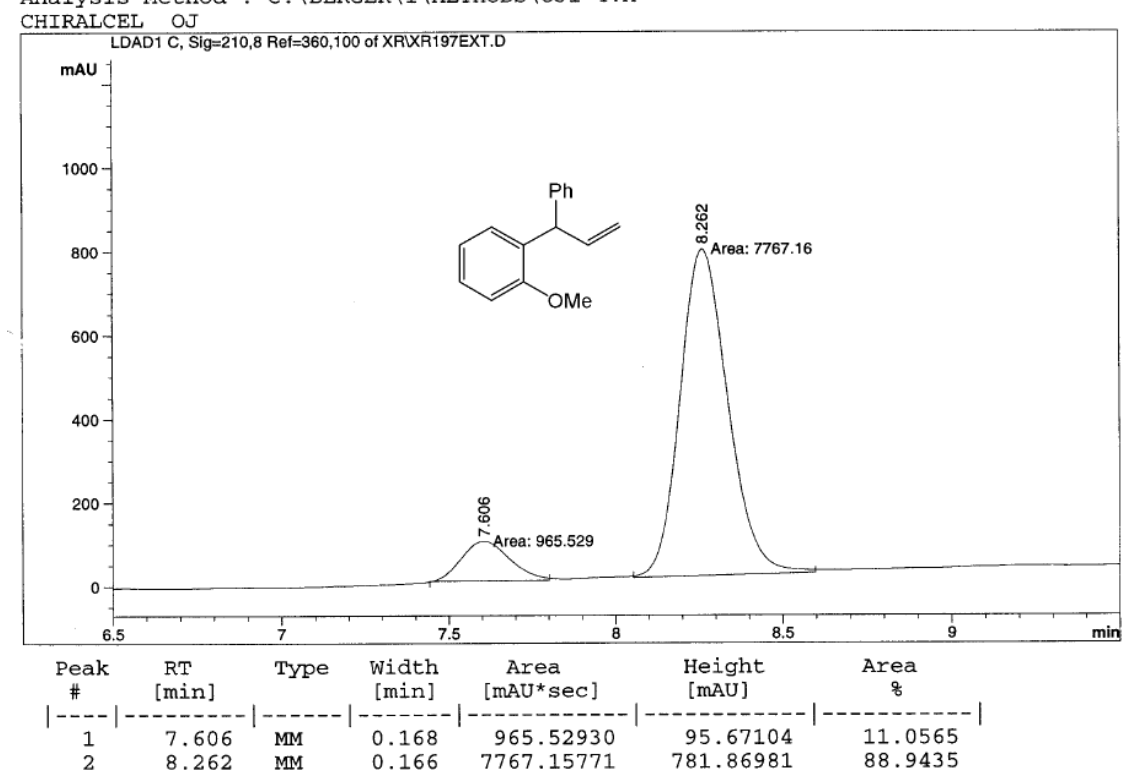


${ }^{1} \mathrm{H}-\mathrm{NMR}$

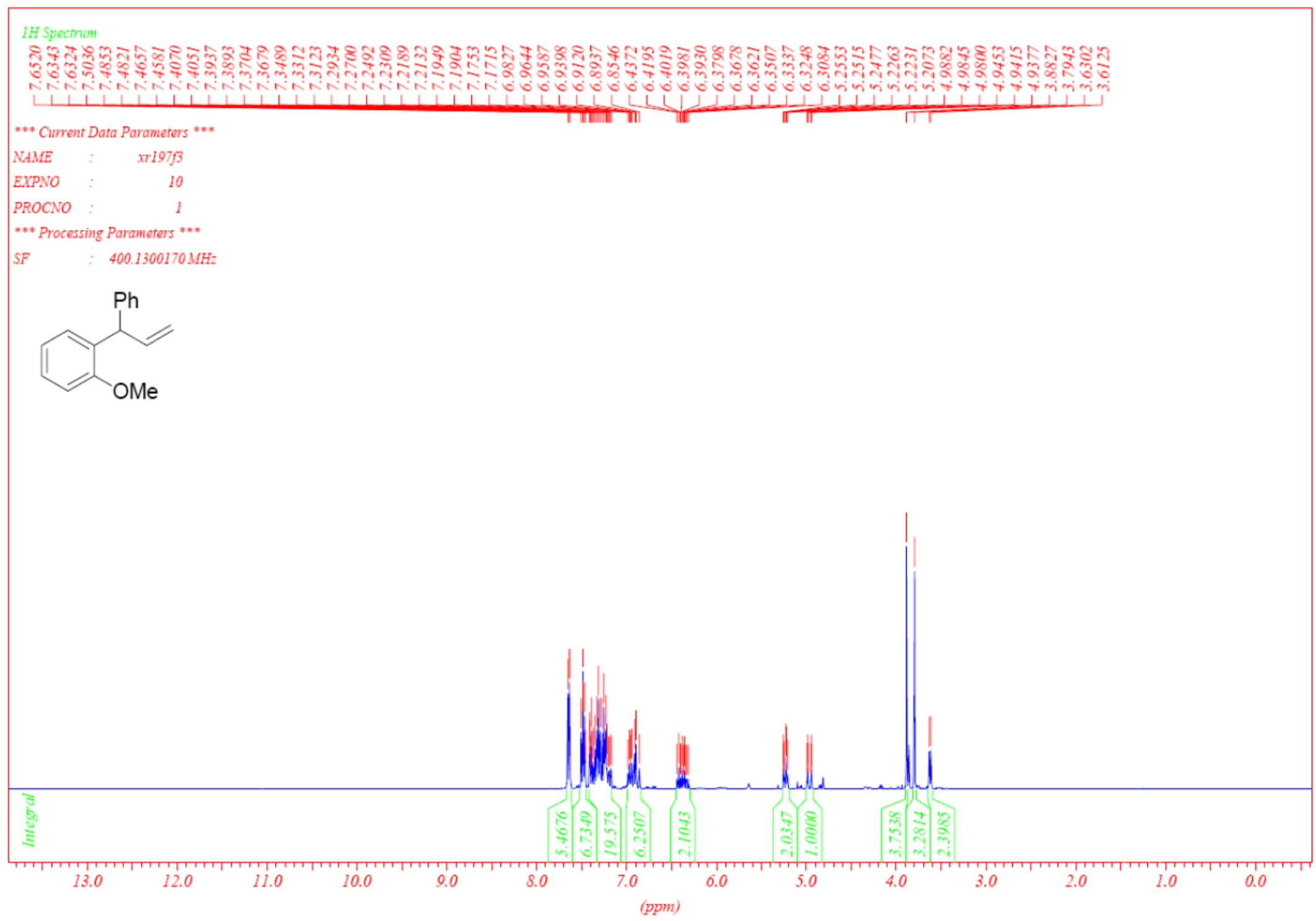


1-methoxy-3-(1-phenylallyl)benzene 6:

The reaction proceeds on $0.5 \mathrm{mmol}$ scale of starting material 5 following GP/NS1 and using ligand L1 (R,R,R). The product was purified by flash column chromatography (silica, pentane), to afford the SN2'-SN2 mixture (52/48) as a

slightly yellow liquid (75.3 mg, 67\% yield). Ee of 90\% was measured by chiral SFC with a AD-H column (program: 2\% MeOH-2'-1-15\%, 200 bar, $\left.2 \mathrm{ml} / \mathrm{min}, 30^{\circ} \mathrm{C}\right) . \mathrm{R}_{\mathrm{T}}$ : $3.14(\mathrm{R})$ and $3.65(\mathrm{~S}) .{ }^{1} \mathrm{H}$ NMR $\left(400 \mathrm{MHz}, \mathrm{CDCl}_{3}\right) \delta$ 6.70-7.70 (m, 9H), $6.35(\mathrm{~m}, 1 \mathrm{H}),, 5.27(\mathrm{~d}, 1 \mathrm{H}, \mathrm{J}=8.0 \mathrm{~Hz}), 5.06$ $(\mathrm{d}, 1 \mathrm{H}, \mathrm{J}=13.6 \mathrm{~Hz}), 4.75(\mathrm{~d}, 1 \mathrm{H}, \mathrm{J}=5.7 \mathrm{~Hz}), 3.81(\mathrm{~s}, 3 \mathrm{H})$.

AD-H $\quad 28-2-1-158-0 \quad 2 \mathrm{ml} / \mathrm{min} . \quad 200 \mathrm{Bar}$

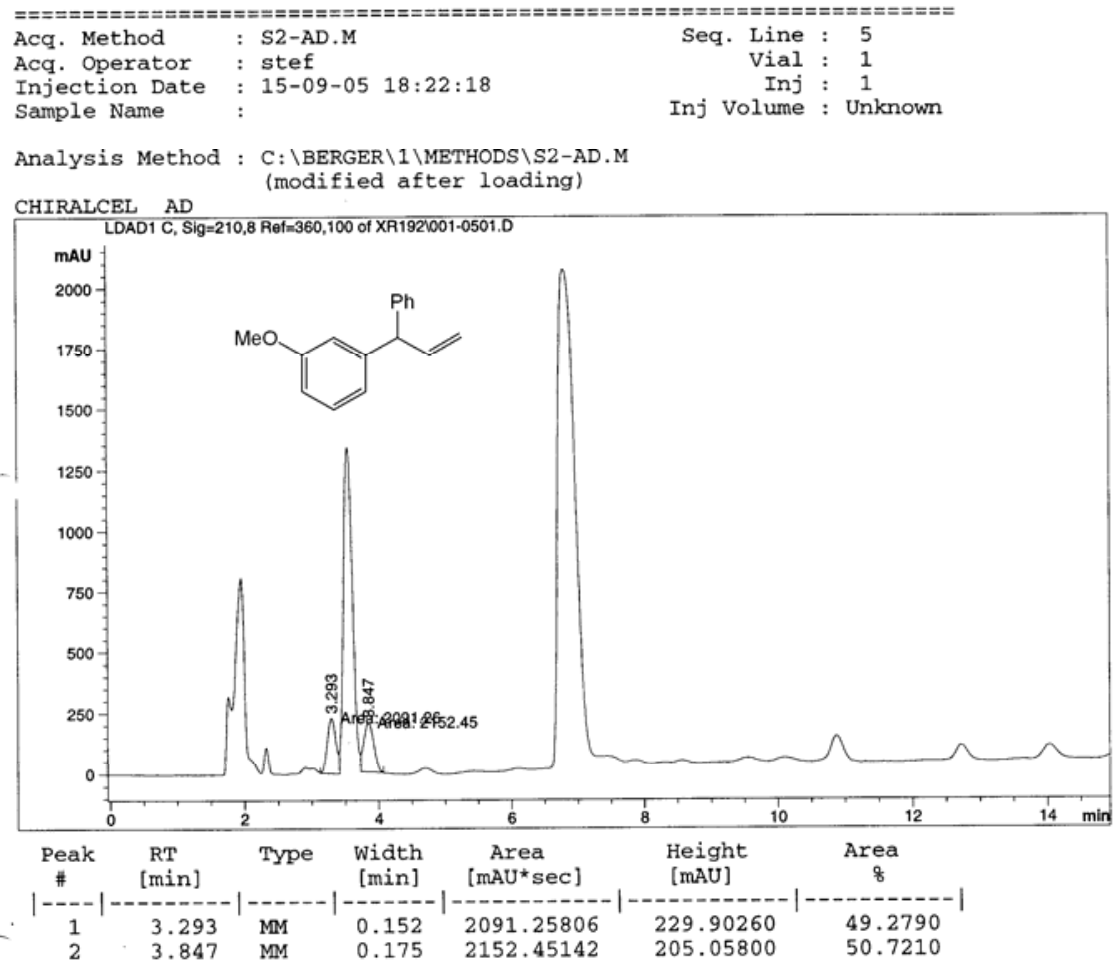

AD-H 2-2-1-15-0 200 bars $2 \mathrm{ml} / \mathrm{min} 30 \mathrm{C}$

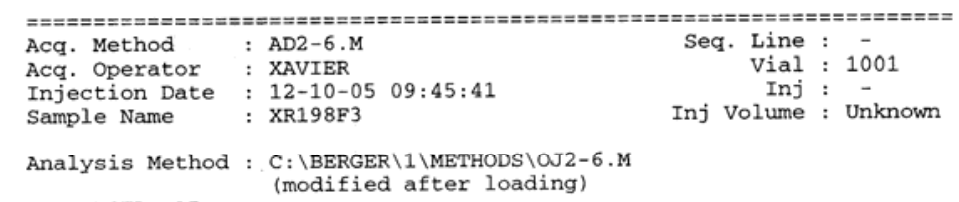
CHIRALCEL AD

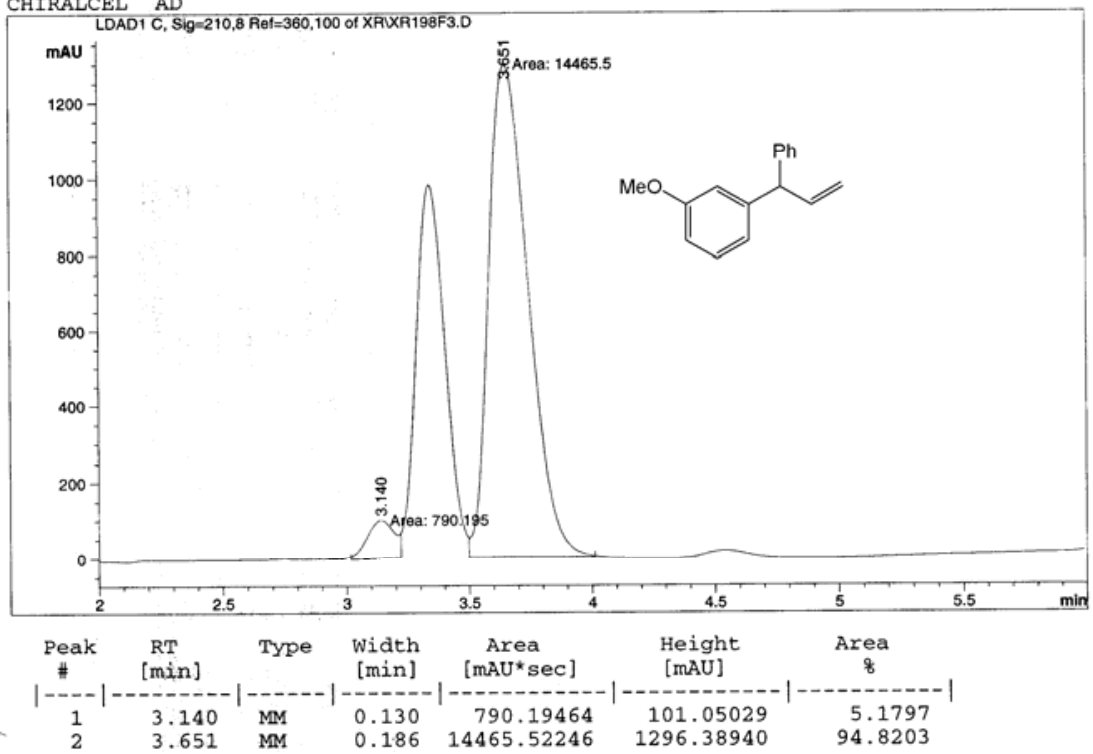


${ }^{1} \mathrm{H}-\mathrm{NMR}$

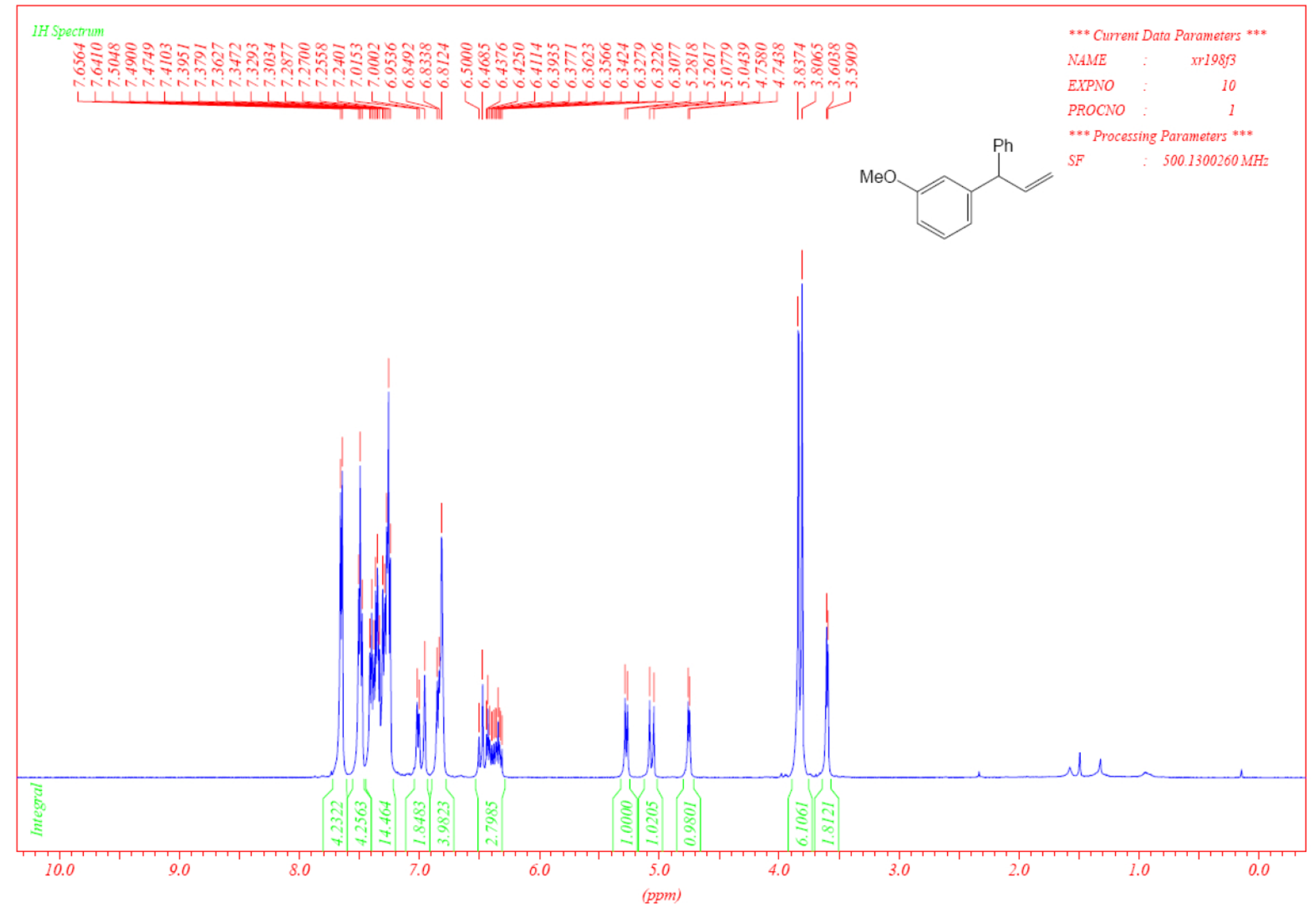


${ }^{13} \mathrm{C}-\mathrm{NMR}$

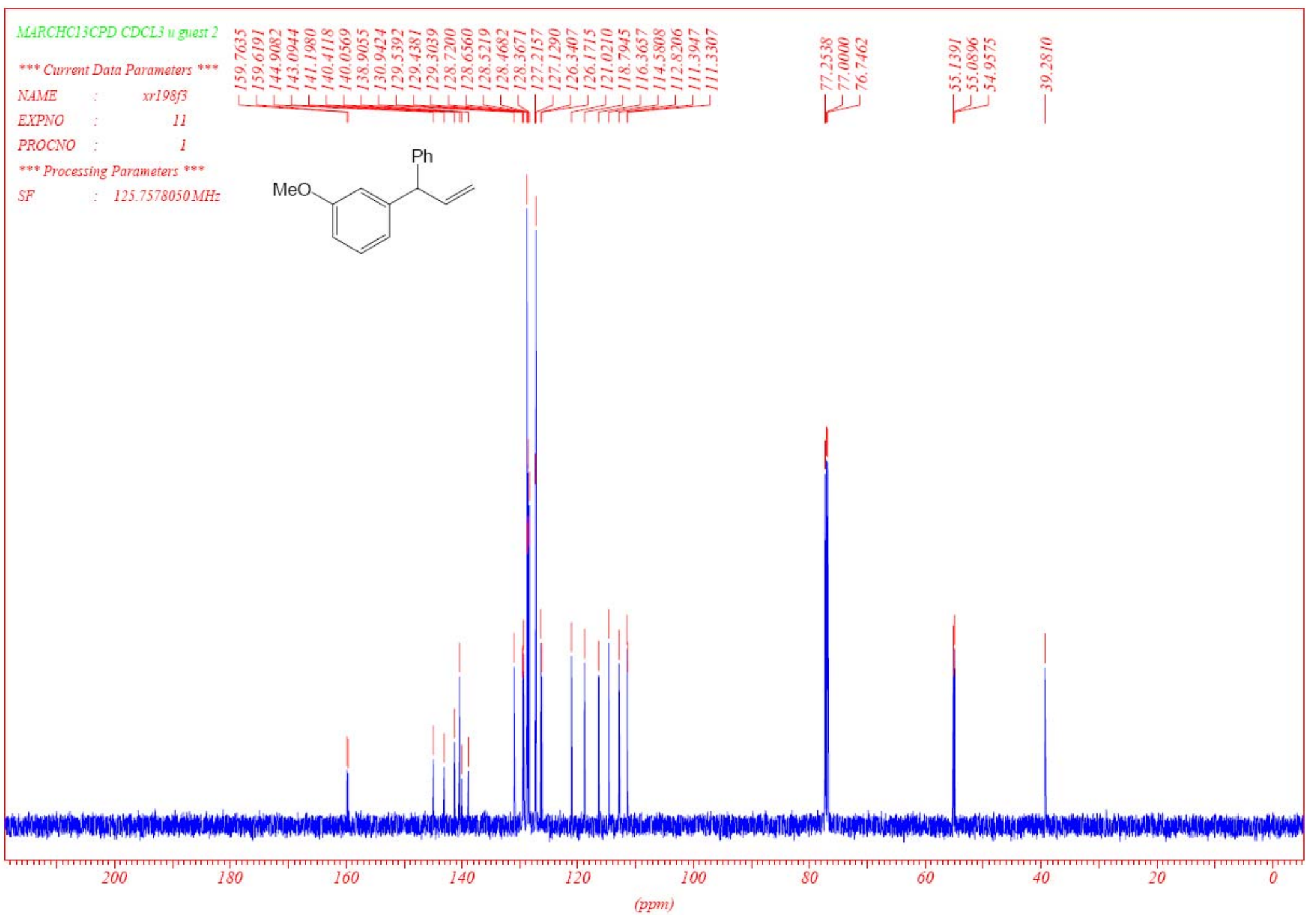




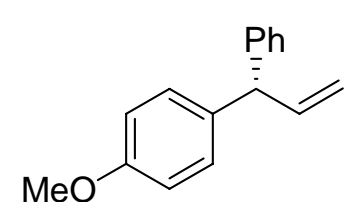

\section{1-(1-(4-methoxyphenyl)allyl)benzene 8:}

The reaction proceeds on $0.5 \mathrm{mmol}$ scale of starting material 7 following GP/NS1 and using ligand L1 (R,R,R). The product was purified by flash column chromatography (silica, pentane), to afford the SN2'-SN2 mixture as a slightly

yellow liquid (68.8 mg, 61\% yield). Ee of 91\% was measured by chiral SFC with a OJ-H column (program: 1\% MeOH-4'-1-20\%, 200 bar, 2 $\left.\mathrm{ml} / \mathrm{min}, 30^{\circ} \mathrm{C}\right) . \mathrm{R}_{\mathrm{T}}: 10.64(\mathrm{~S})$ and $12.58(\mathrm{R}) . \delta 6.80-7.70(\mathrm{~m}, 9 \mathrm{H}), 6.34(\mathrm{~m}, 1 \mathrm{H}),, 5.26(\mathrm{~d}, 1 \mathrm{H}, \mathrm{J}=10.1 \mathrm{~Hz}), 5.02(\mathrm{~d}, 1 \mathrm{H}, \mathrm{J}=16.9 \mathrm{~Hz}), 4.74(\mathrm{~d}, 1 \mathrm{H}$, $\mathrm{J}=7.3 \mathrm{~Hz}), 3.83$ (s, 3H).

OJ 18-4-1-208 200 bars $2 \mathrm{ml} / \mathrm{min} \quad 30 \mathrm{C}$

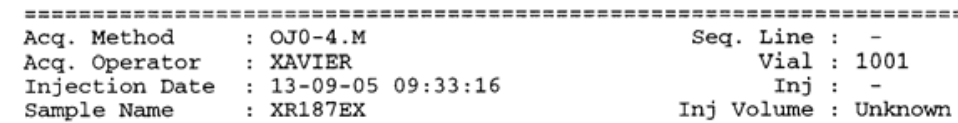

Analysis Method : $\begin{aligned} & C: \backslash \text { BERGER } \backslash 1 \backslash \text { METHODS } \backslash \text { OJO-4.M } \\ & \text { (modified after loading) }\end{aligned}$

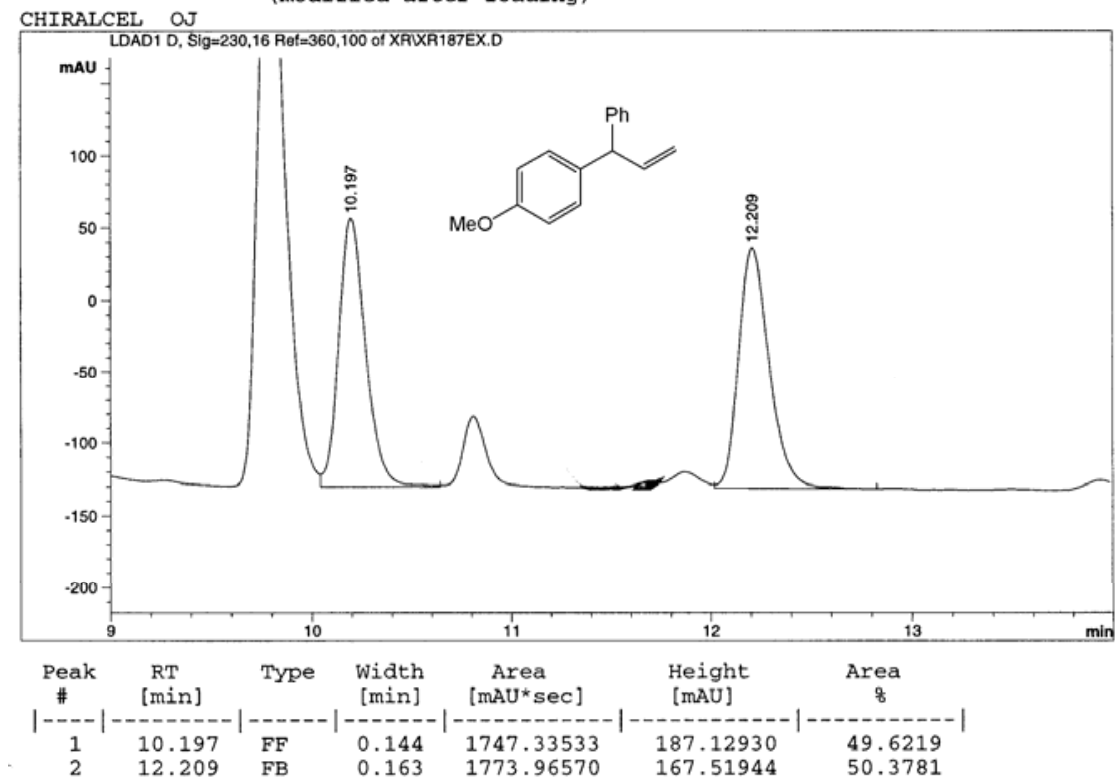

OJ-H $\quad 18-4-1-208 \quad 200$ bars $2 \mathrm{ml} / \mathrm{min} \quad 30 \mathrm{C}$

\begin{tabular}{|c|c|c|}
\hline $\begin{array}{l}\text { Acq. Method } \\
\text { Acq. Operator } \\
\text { Injection Date } \\
\text { Sample Name }\end{array}$ & $\begin{array}{l}: \text { OJ1-4.M } \\
: \text { XAVIER } \\
: 21-09-05 \quad 11: 37: 07 \\
: \text { XR199EXT }\end{array}$ & $\begin{array}{r}\text { Seq. Line : } \\
\text { Vial : } 1001 \\
\text { Inj } \\
\text { Inj Volume }\end{array}$ \\
\hline alysis Me & $\begin{array}{l}\text { BERGER } 1 \backslash M E T \\
\text { dified after }\end{array}$ & \\
\hline
\end{tabular}
CHIRALCEL OU

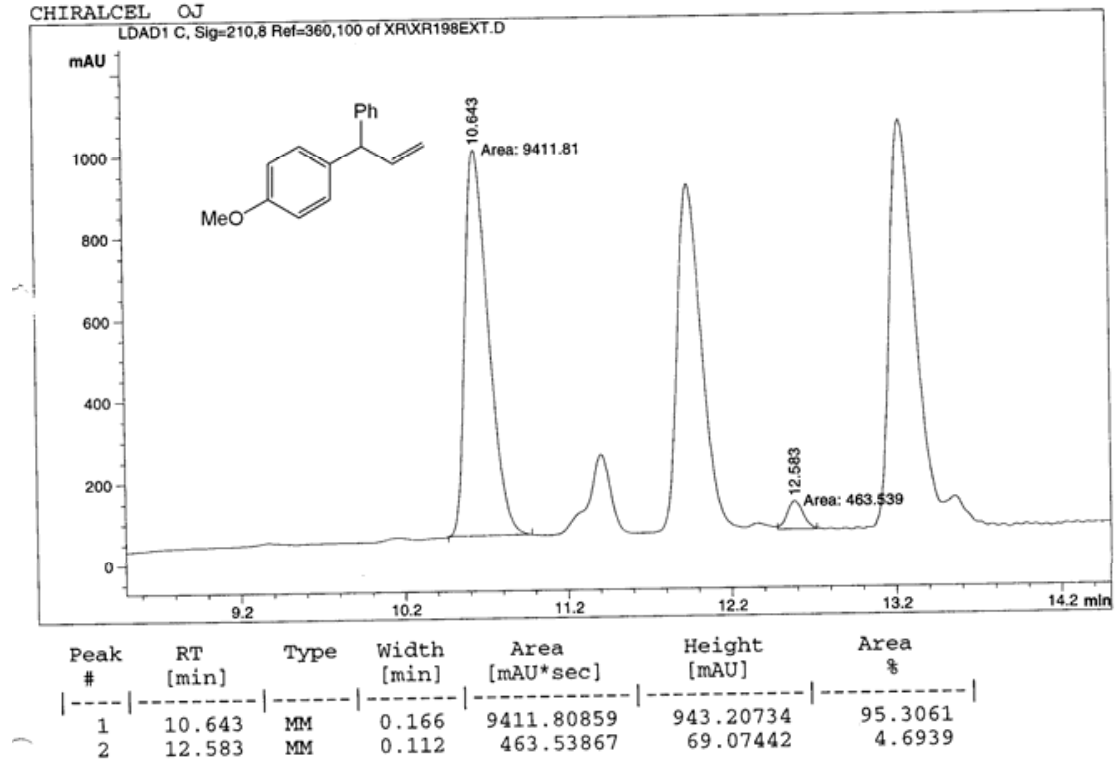




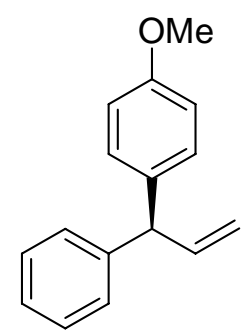

\section{1-(1-(4-methoxyphenyl)allyl)benzene 8:}

The reaction proceeds on $0.5 \mathrm{mmol}$ scale of starting material 21 following GP/NS1 and using ligand L1 (S,S,S). The product was purified by flash column chromatography (silica, pentane/ $\mathrm{Et}_{2} \mathrm{O}$ 9/1), to afford the SN2'-SN2 mixture (63/37) as a slightly yellow liquid (112.0 mg, 100\% yield). Ee of $91 \%$ was measured by chiral SFC with a OJ-H column (program: $\left.1 \% \mathrm{MeOH}-44^{\prime}-1-20 \%, 200 \mathrm{bar}, 2 \mathrm{ml} / \mathrm{min}, 30^{\circ} \mathrm{C}\right) . \mathrm{R}_{\mathrm{T}}: 11.38(\mathrm{~S})$ and $13.59(\mathrm{R}) .{ }^{1} \mathrm{H} \mathrm{NMR}\left(400 \mathrm{MHz}, \mathrm{CDCl}_{3}\right) \delta 6.80-7.70(\mathrm{~m}$, 9H), $6.34(\mathrm{~m}, 1 \mathrm{H}),, 5.26(\mathrm{~d}, 1 \mathrm{H}, \mathrm{J}=10.1 \mathrm{~Hz}), 5.02(\mathrm{~d}, 1 \mathrm{H}, \mathrm{J}=16.9 \mathrm{~Hz}), 4.74(\mathrm{~d}, 1 \mathrm{H}, \mathrm{J}=7.3 \mathrm{~Hz}), 3.83(\mathrm{~s}, 3 \mathrm{H})$.

OJ $18-4-1-20 \% \quad 200$ bars $2 \mathrm{ml} / \mathrm{min} \quad 30 \mathrm{C}$

\begin{tabular}{|c|c|c|}
\hline Acq. Method & : OJO-4.M & Line : \\
\hline Acq. Operator & $\therefore$ XAVIER & Vial : 1001 \\
\hline $\begin{array}{l}\text { Injection Date } \\
\text { Sample Name }\end{array}$ & $\begin{array}{l}: 13-09-05 \quad 09: 33: 16 \\
: \text { XR187EX }\end{array}$ & $\begin{aligned} \text { Inj } & - \\
\text { Inj Volume } & -\end{aligned}$ \\
\hline Analysis Method & $\begin{array}{l}\mathrm{C}: \backslash \mathrm{BERGER} \backslash 1 \backslash \mathrm{METHO} \\
\text { (modified after } 1\end{array}$ & \\
\hline
\end{tabular}

CHIRALCEL OJ (modified after loading)

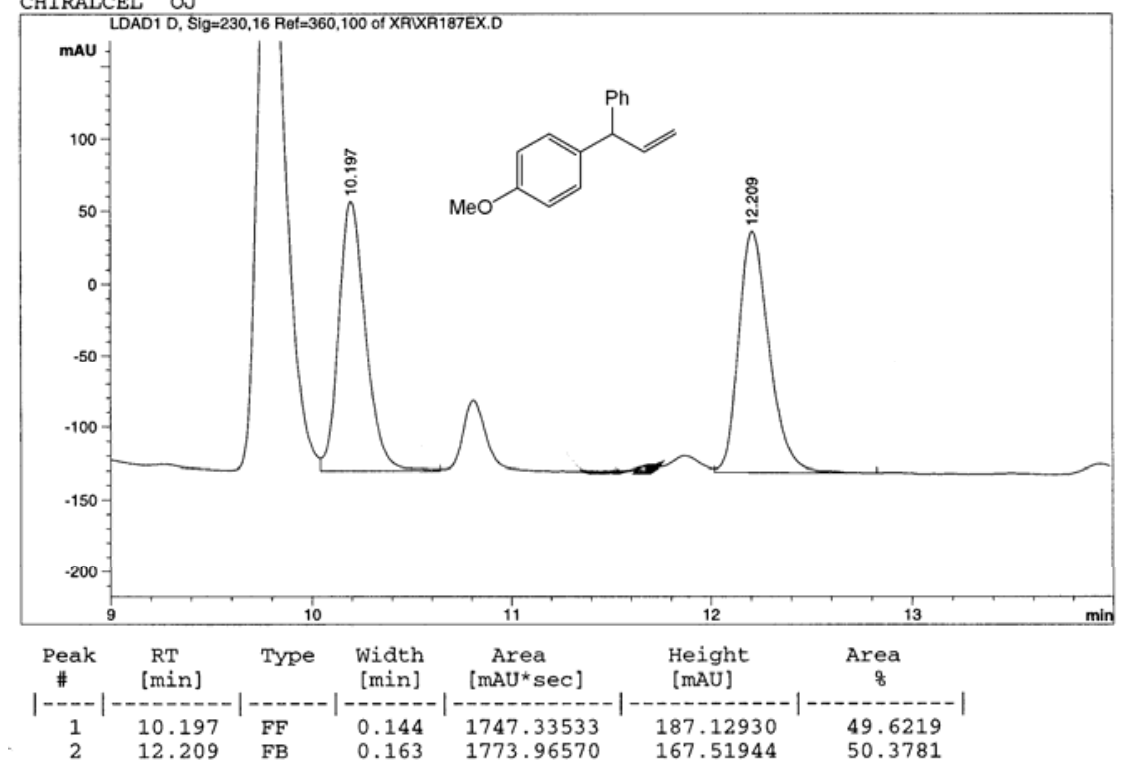

OJ-H 18-4-1-208 200 bars $2 \mathrm{ml} / \mathrm{min} \quad 30 \mathrm{C}$

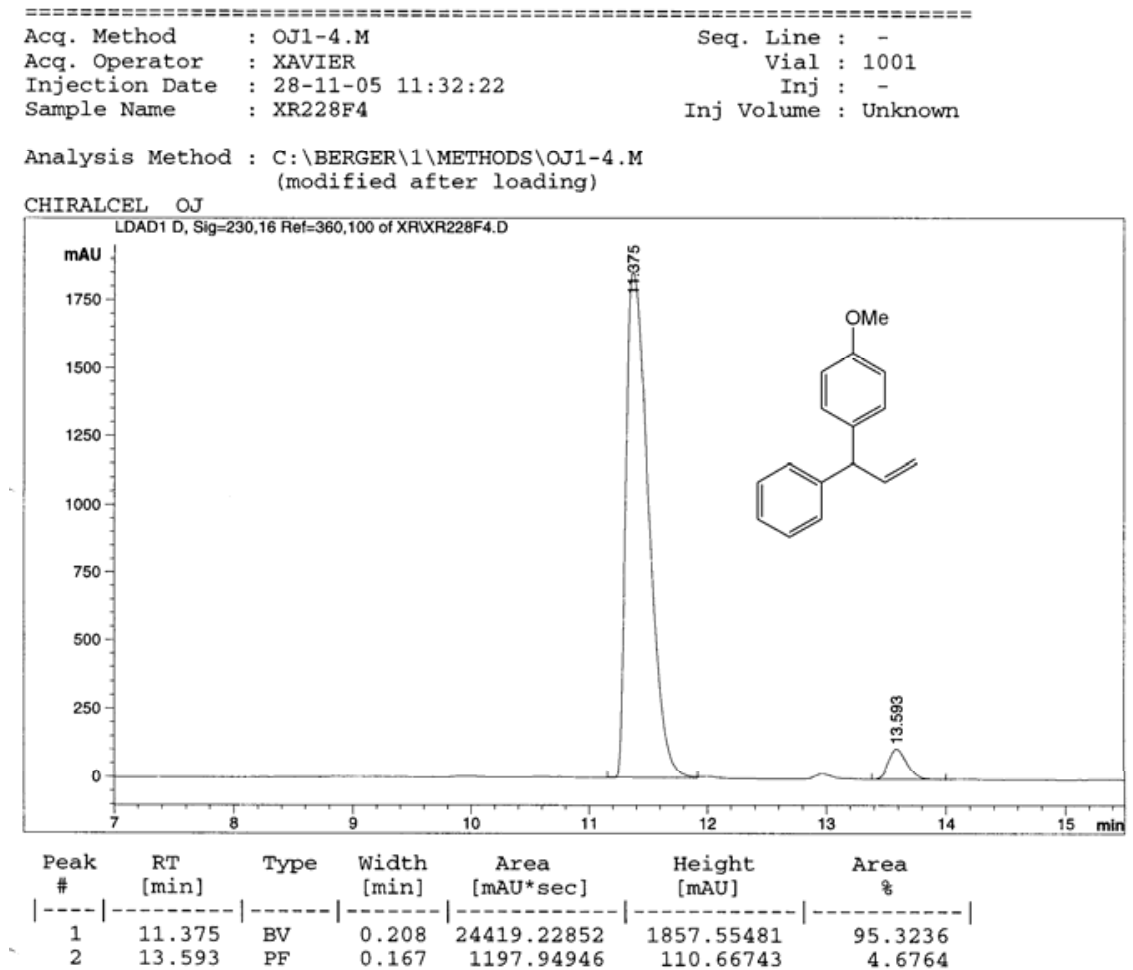


${ }^{1} \mathrm{H}-\mathrm{NMR}$

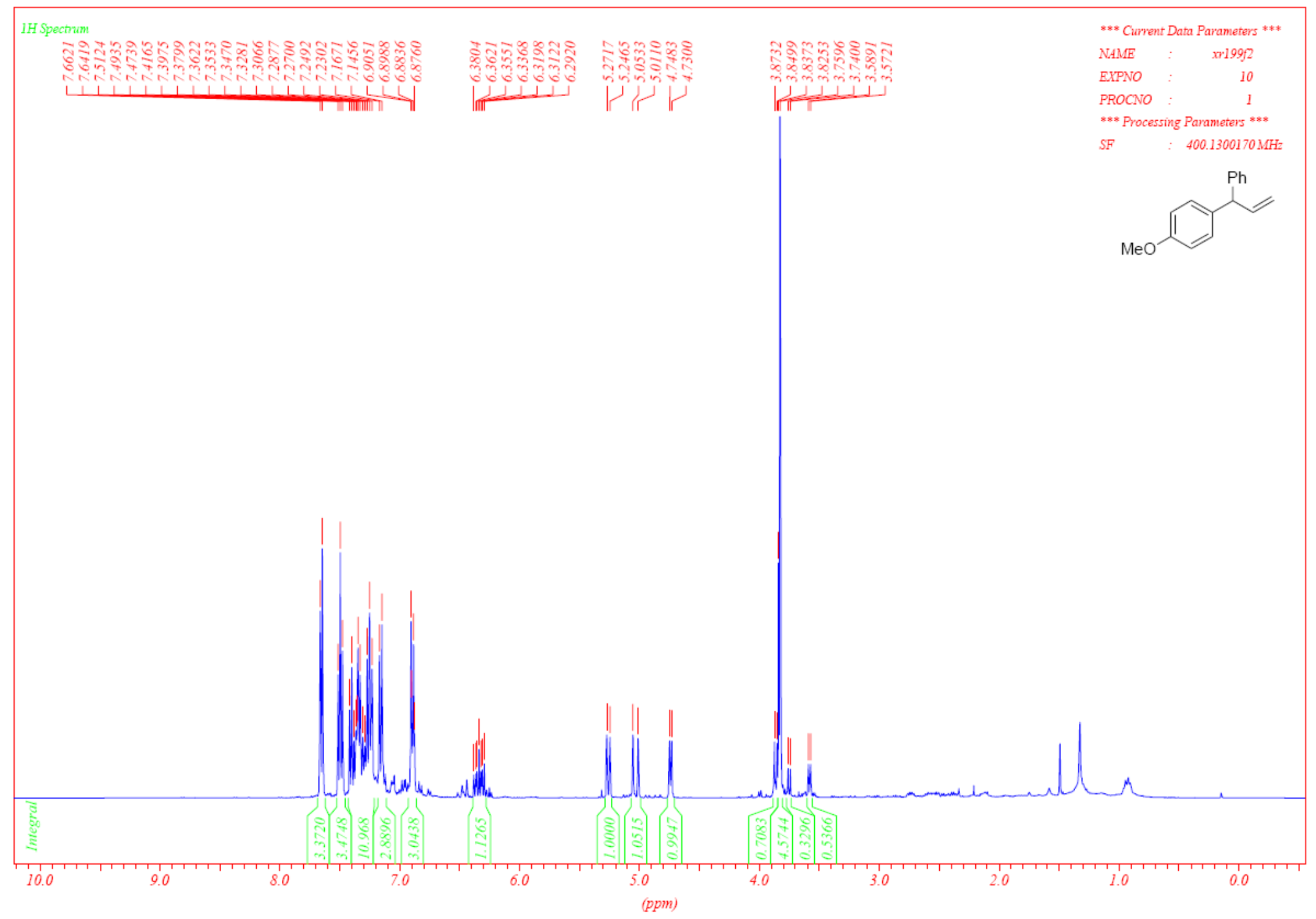




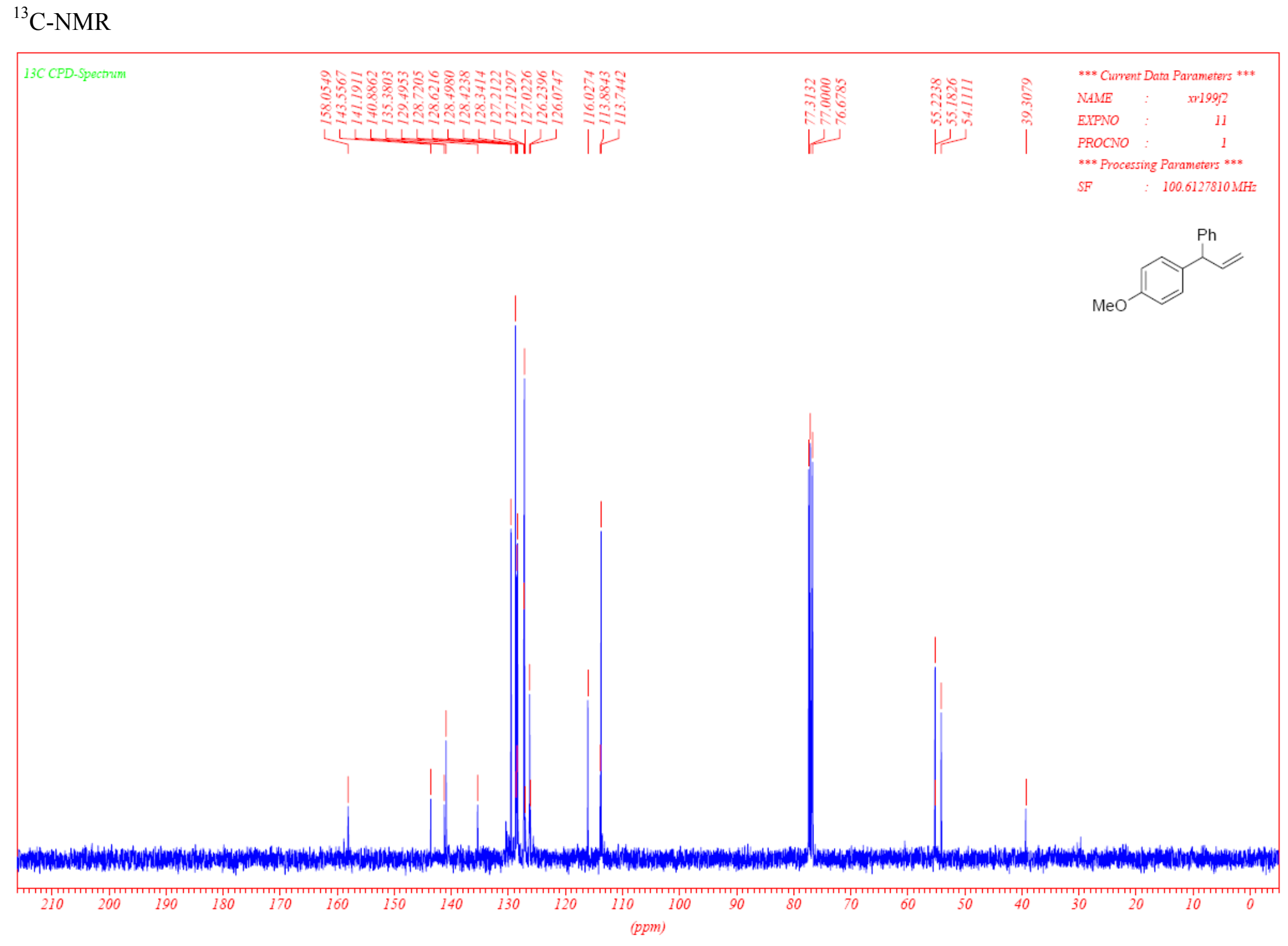




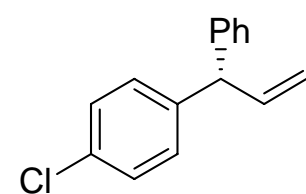

\section{1-(1-(4-chlorophenyl)allyl)benzene 10:}

The reaction proceeds on $0.5 \mathrm{mmol}$ scale of starting material 9 following GP/NS1 and using ligand L1 (R,R,R). The product was purified by flash column chromatography (silica, pentane), to afford the SN2'-SN2 mixture (55/45) as a colourless liquid (94.6 mg, 83\% yield). Ee of 99.2\% was measured by chiral SFC with a OJ-H column (program: 5\% MeOH-2'-1-15\%, 200 bar, $2 \mathrm{ml} / \mathrm{min}$, $\left.20^{\circ} \mathrm{C}\right) . \mathrm{R}_{\mathrm{T}}: 5.80(\mathrm{R})$ and $7.58(\mathrm{~S}) . \delta$ 7.10-7.70 (m, 9H), $6.32(\mathrm{~m}, 1 \mathrm{H}),, 5.30(\mathrm{~d}, 1 \mathrm{H}, \mathrm{J}=10.1 \mathrm{~Hz}), 5.05(\mathrm{~d}, 1 \mathrm{H}, \mathrm{J}=16.9 \mathrm{~Hz}), 4.76(\mathrm{~d}, 1 \mathrm{H}, \mathrm{J}=7.0$ $\mathrm{Hz})$.

OJ-H $5-2-1-158 \quad 200$ bars $2 \mathrm{ml} / \mathrm{min} \quad 20 \mathrm{C}$

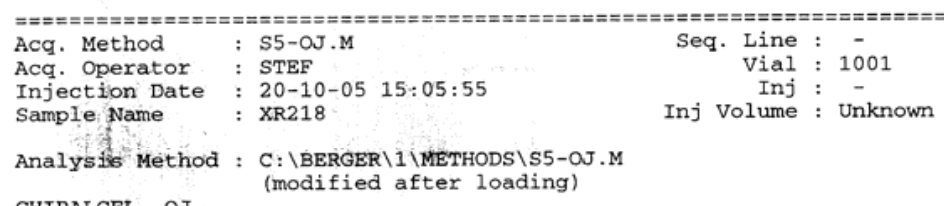

ALCEL OJ Modified after loading

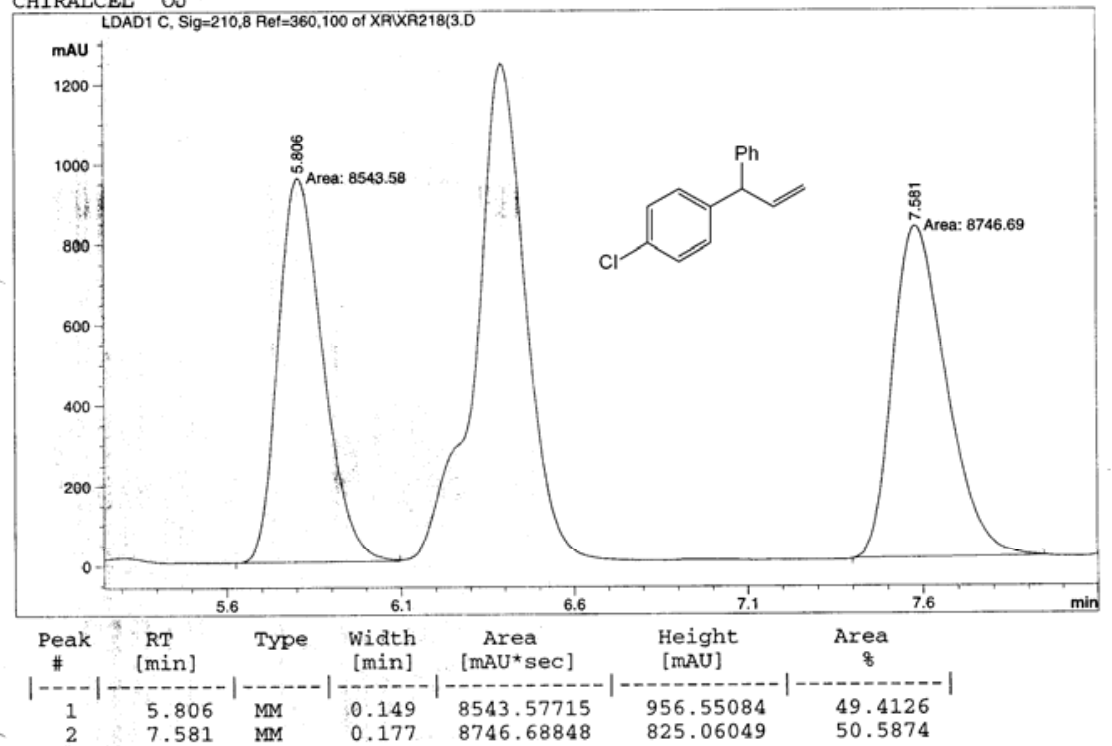

of $\rightarrow$ H $5 \%-2-15 \%-0200$ bars $2 \mathrm{ml} / \mathrm{min} \quad 20 \mathrm{C}$

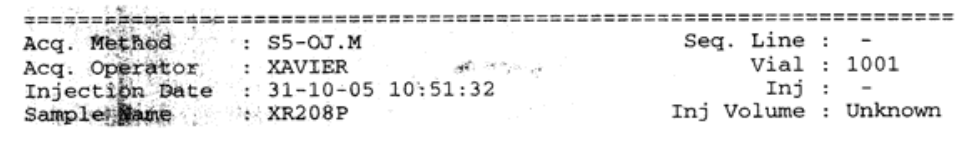

Analysa thoed : $C: \backslash$ BERGER $1 \backslash$ MBTHODSAS5-OJ.M

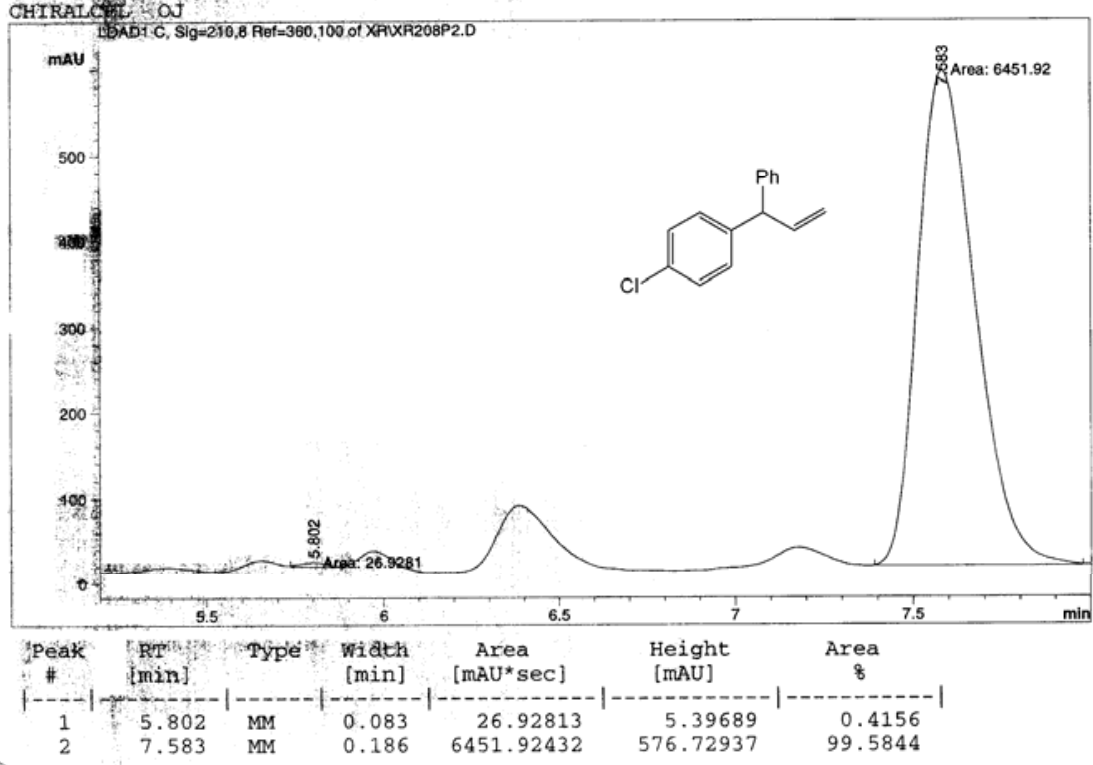


${ }^{1} \mathrm{H}-\mathrm{NMR}$

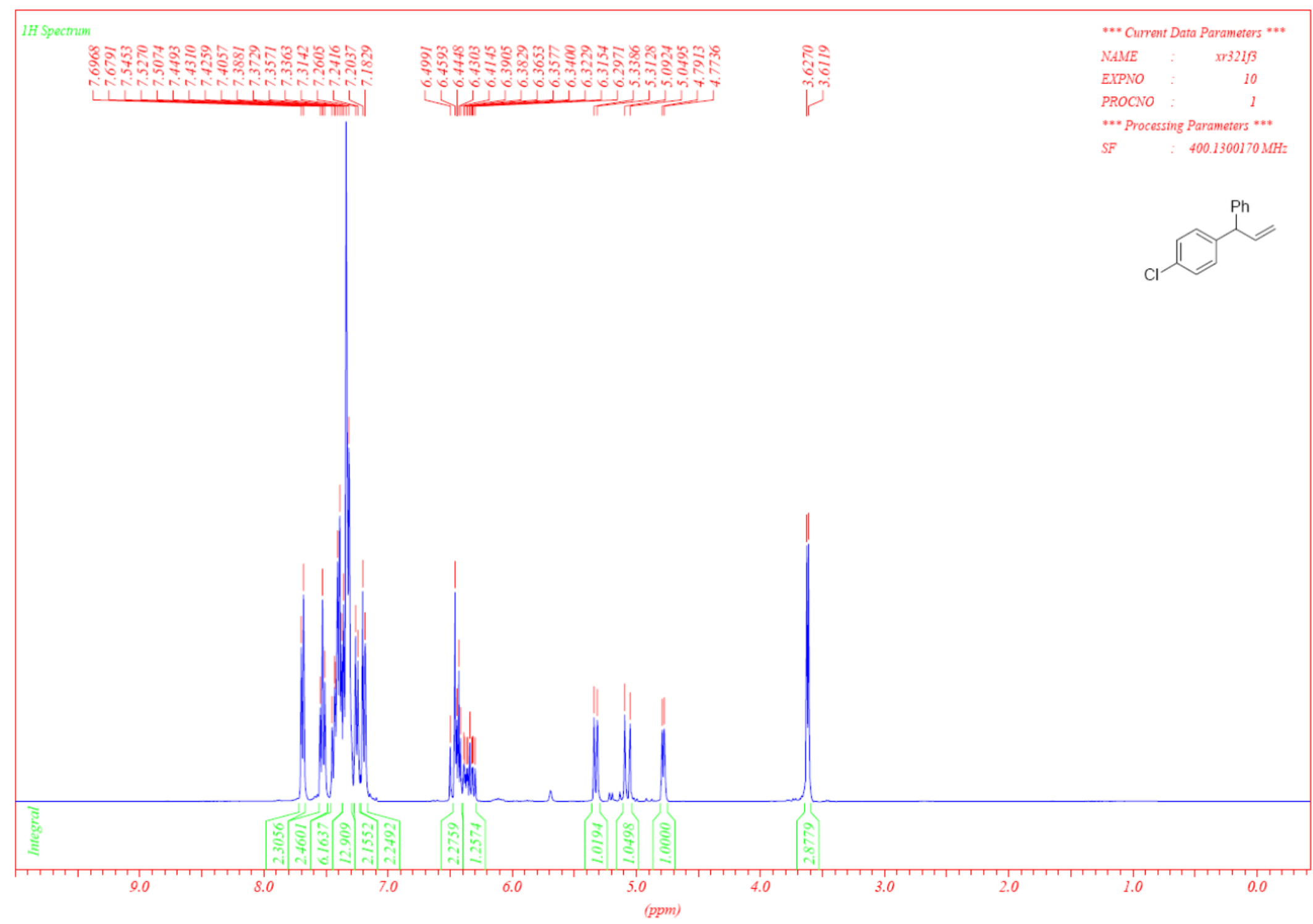


${ }^{13} \mathrm{C}-\mathrm{NMR}$

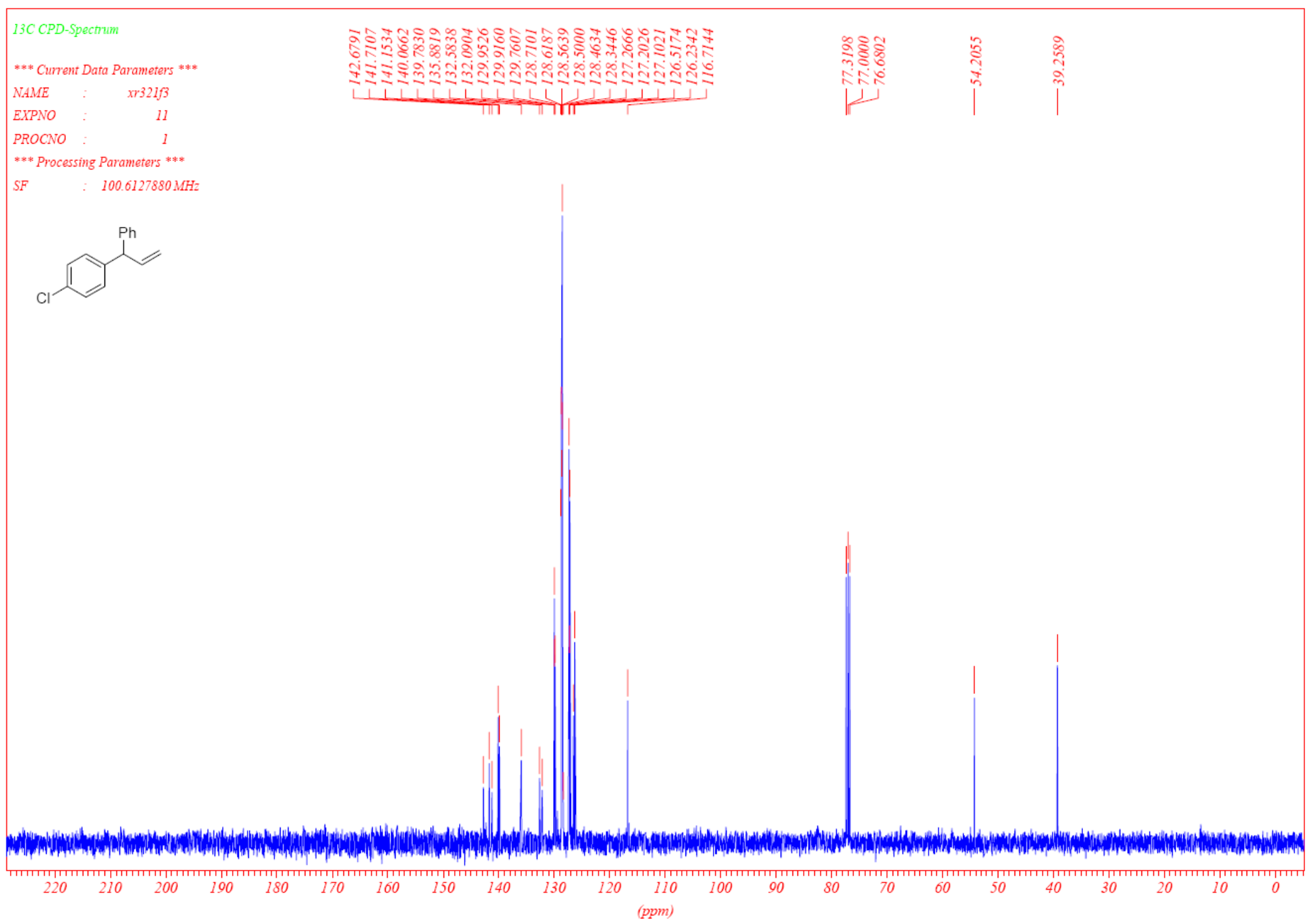

S17 


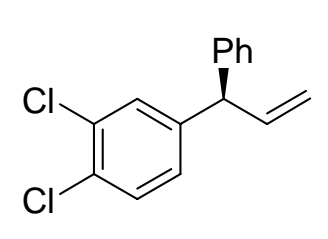

\section{1,2-dichloro-4-(1-phenylallyl)benzene 12:}

The reaction proceeds on 4.0 mmol scale of starting material 11 following GP/NS1 and using ligand L1 (S,S,S). The product was purified by flash column chromatography (silica, pentane), to afford $551.2 \mathrm{mg}$ (52\% yield) of desired compound $\mathbf{1 2}$ as a colourless liquid and $510 \mathrm{mg}$ (48\% yield) of SN2'-SN2 mixture. Ee of $96 \%$ was measured by chiral SFC with a OJ-H column (program: 2\% MeOH-1'-2-15\%, $\left.175 \mathrm{bar}, 2 \mathrm{ml} / \mathrm{min}, 30^{\circ} \mathrm{C}\right) . \mathrm{R}_{\mathrm{T}}: 6.25(\mathrm{~S})$ and $6.53(\mathrm{R}) .[\alpha]_{\mathrm{D}}{ }^{20}=+3.0\left(\mathrm{c}=1.00, \mathrm{CHCl}_{3}\right){ }^{1} \mathrm{H} \mathrm{NMR}(400$ $\left.\mathrm{MHz}_{\mathrm{CDCl}}\right) \delta$ 6.80-7.80 (m, 8H), $6.27(\mathrm{~m}, 1 \mathrm{H}),, 5.30(\mathrm{dd}, 1 \mathrm{H}, \mathrm{J}=10.4 \mathrm{~Hz}, \mathrm{~J}=1.0 \mathrm{~Hz}), 5.06(, 1 \mathrm{H}, \mathrm{J}=\mathrm{Hz}), 4.71(\mathrm{~d}, 1 \mathrm{H}, \mathrm{J}=7.1 \mathrm{~Hz}) .{ }^{13} \mathrm{C} \mathrm{NMR}$ $\left(100 \mathrm{MHz}, \mathrm{CDCl}_{3}\right) \delta 143.5,142.0,139.4,132.3,130.5,130.3,130.2,128.6(2 \mathrm{C}), 128.4(2 \mathrm{C}), 128.0,126.8,117.3,54.0$.

OJ-H 28-1-2-15\% 175 bars $2 \mathrm{ml} / \mathrm{min} \quad 30 \mathrm{C}$
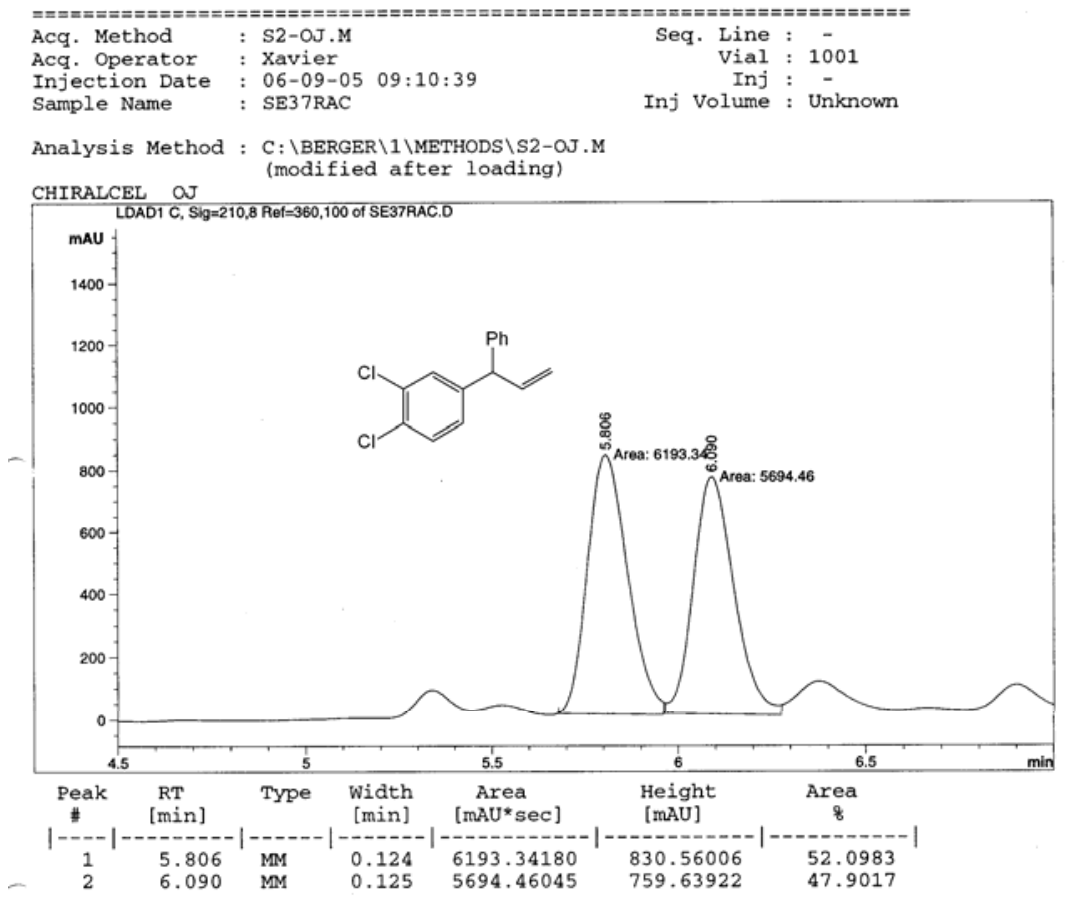

OJ-H 28-1-2-158 175 bars $2 \mathrm{~mL} / \mathrm{min} \quad 30 \mathrm{C}$

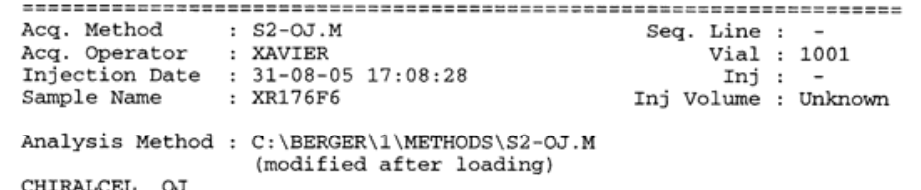
ified after loading)

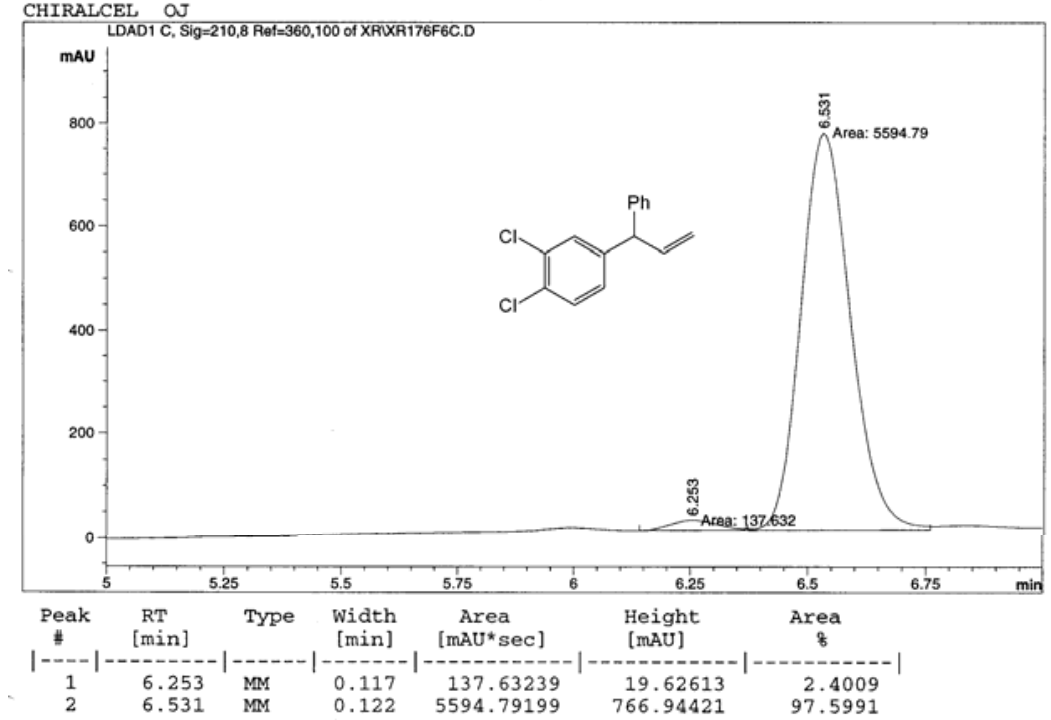




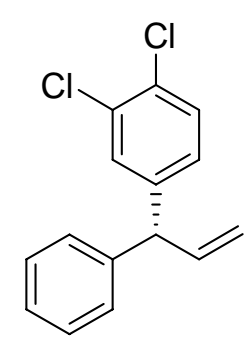

\section{1,2-dichloro-4-(1-phenylallyl)benzene 12:}

The reaction proceeds on $0.5 \mathrm{mmol}$ scale of starting material 21 following GP/NS1 and using ligand L1 (R,R,R). Crude product was not purified. Ee of 63\% was measured by chiral SFC with a OJ-H column (program: 2\% MeOH-1'-2-15\%, 175 bar, 2 $\left.\mathrm{ml} / \mathrm{min}, 30^{\circ} \mathrm{C}\right) . \mathrm{R}_{\mathrm{T}}: 6.25(\mathrm{~S})$ and $6.53(\mathrm{R}) .{ }^{1} \mathrm{H}$ NMR $\left(400 \mathrm{MHz}, \mathrm{CDCl}_{3}\right) \delta 6.80-7.80(\mathrm{~m}, 8 \mathrm{H}), 6.27(\mathrm{~m}, 1 \mathrm{H}),, 5.30(\mathrm{dd}, 1 \mathrm{H}, \mathrm{J}=10.4$ $\mathrm{Hz}, \mathrm{J}=1.0 \mathrm{~Hz}), 5.06(, 1 \mathrm{H}, \mathrm{J}=\mathrm{Hz}), 4.71(\mathrm{~d}, 1 \mathrm{H}, \mathrm{J}=7.1 \mathrm{~Hz}) .{ }^{13} \mathrm{C}$ NMR $\left(100 \mathrm{MHz}, \mathrm{CDCl}_{3}\right) \delta 143.5,142.0,139.4,132.3,130.5$,

$130.3,130.2,128.6(2 \mathrm{C}), 128.4(2 \mathrm{C}), 128.0,126.8,117.3,54.0$.

OJ-H $28-1-2-158 \quad 175$ bars $2 \mathrm{~m} 1 / \mathrm{min} \quad 30 \mathrm{C}$

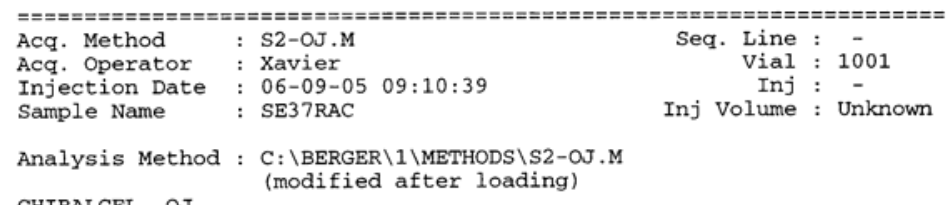

CHIRALCEL OJ (modified after loading)

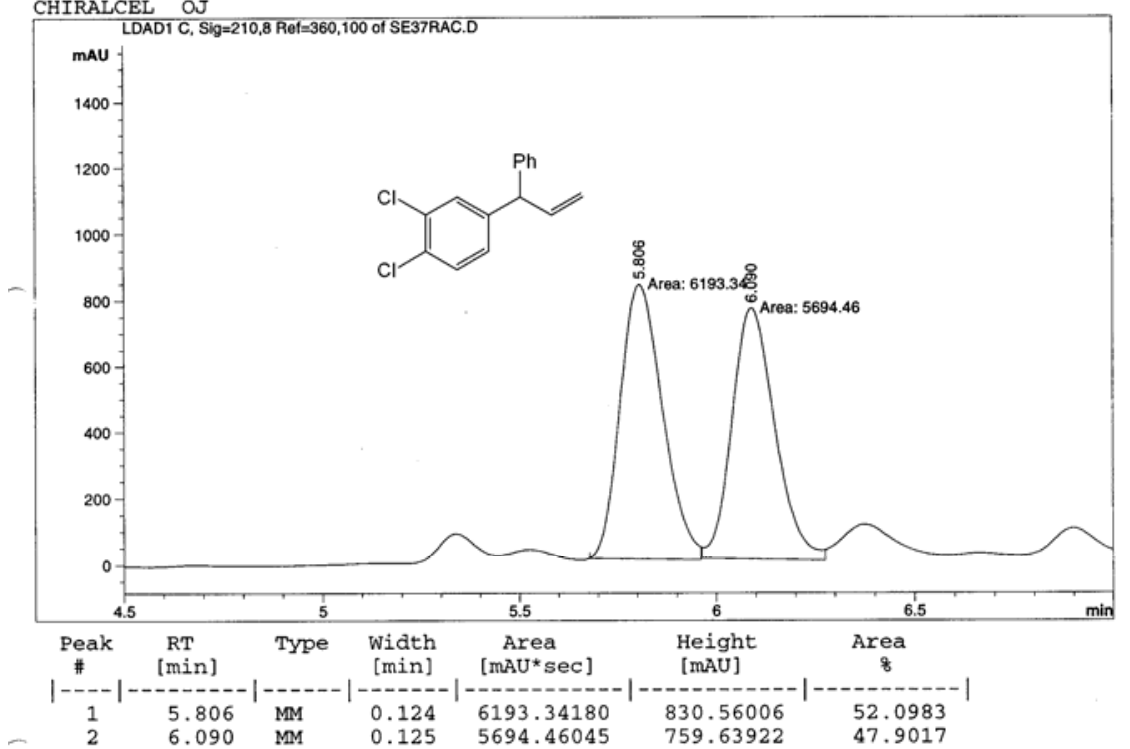

OJ-H 2-1-2-15-O 175 bars $2 \mathrm{ml} / \mathrm{min} 30 \mathrm{C}$

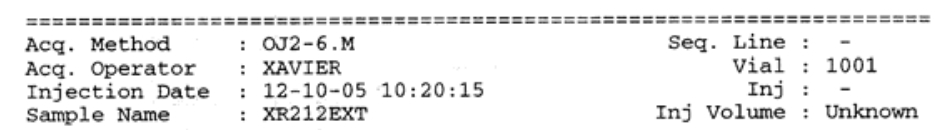

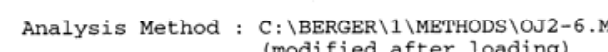

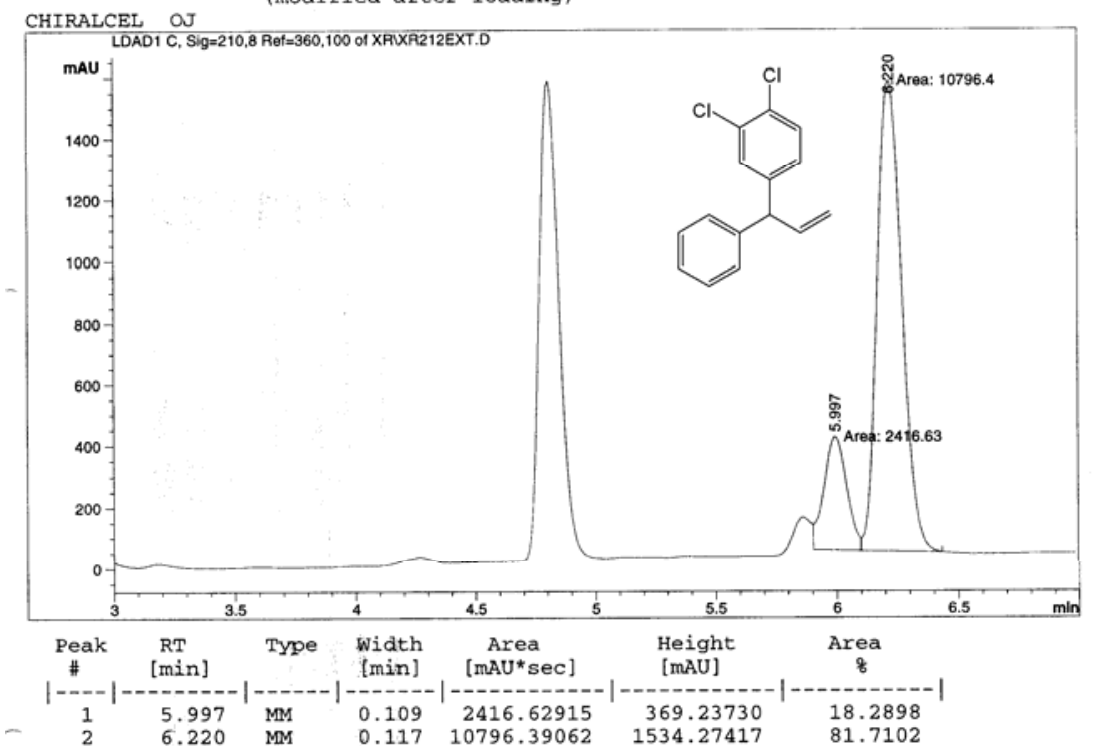


${ }^{1} \mathrm{H}-\mathrm{NMR}$

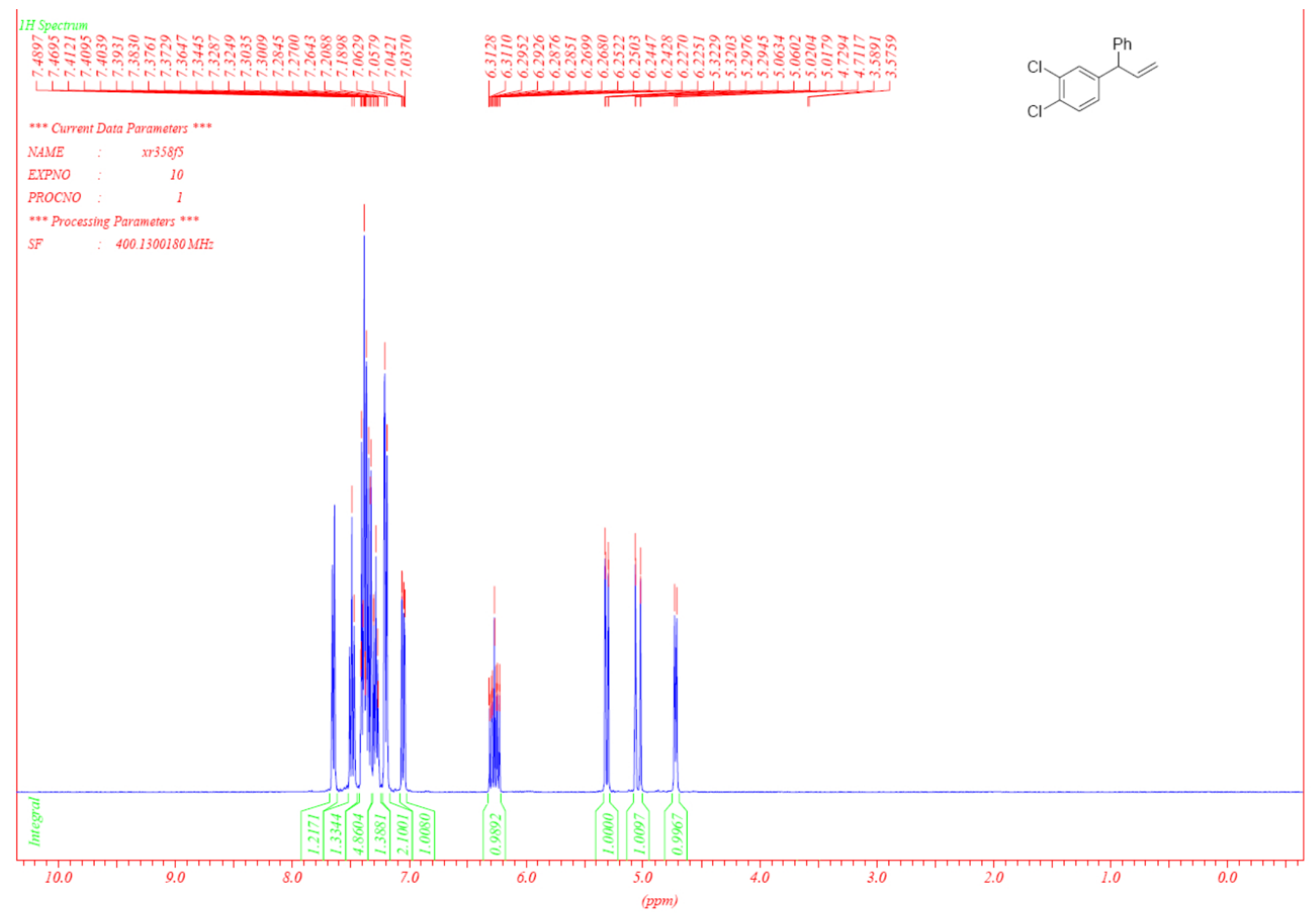

S20 


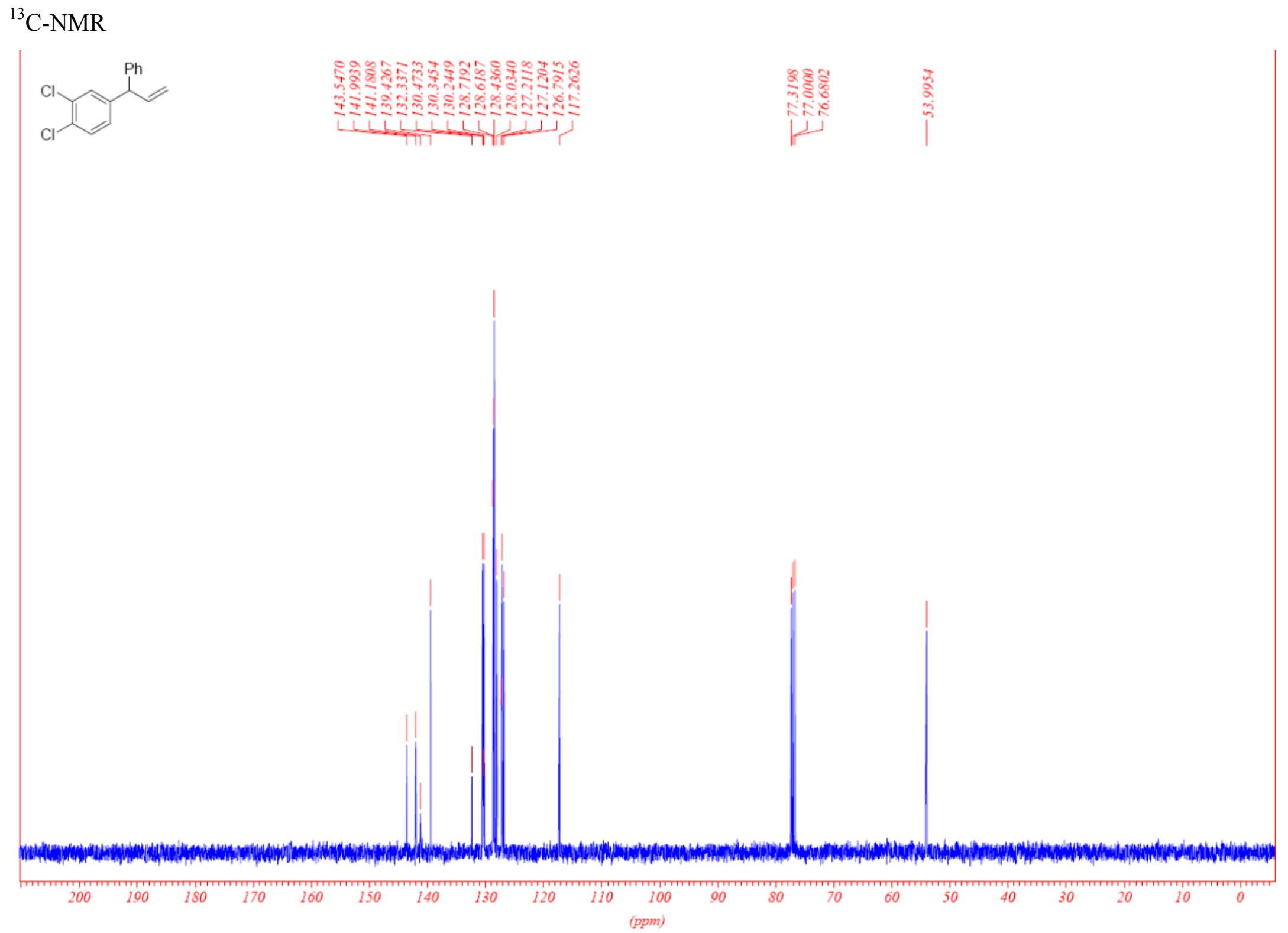




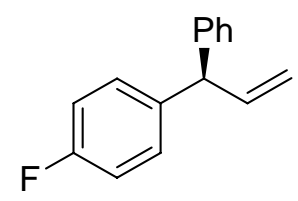

\section{1-(1-(4-fluorophenyl)allyl)benzene 14:}

The reaction proceeds on $0.5 \mathrm{mmol}$ scale of starting material 13 following GP/NS1 and using ligand L1 (S,S,S). The product was purified by flash column chromatography (silica, pentane), to afford $88.1 \mathrm{mg}$ ( $83 \%$ yield) of SN2'-SN2 mixture. Ee of 93\% was measured by chiral SFC with a OJ-H column (program: 2\% MeOH-2'-1-15\%, 200 bar, 2 ml/min, $\left.30^{\circ} \mathrm{C}\right) . \mathrm{R}_{\mathrm{T}}: 5.05(\mathrm{~S})$ and $6.74(\mathrm{R}) .{ }^{1} \mathrm{H}$ $\operatorname{NMR}\left(400 \mathrm{MHz}, \mathrm{CDCl}_{3}\right) \delta$ 6.97-7.53 (m, 9H), $6.29(\mathrm{~m}, 1 \mathrm{H}),, 5.25(\mathrm{~d}, 1 \mathrm{H}, \mathrm{J}=10.1 \mathrm{~Hz}), 4.99(\mathrm{~d}, 1 \mathrm{H}, \mathrm{J}=17.2 \mathrm{~Hz}), 4.73(\mathrm{~d}, 1 \mathrm{H}, \mathrm{J}=7.0 \mathrm{~Hz}) .{ }^{13} \mathrm{C}$ $\operatorname{NMR}\left(100 \mathrm{MHz}, \mathrm{CDCl}_{3}\right) \delta 143.0,140.4,130.04,129.96,128.6,128.49,128.46$ (2C), 126.5, 116.5, 115.8, 115.5, 115.2, $115.1,54.1$.

Og-H $\quad 2 \%-2-1-15 \%-0 \quad 2 \mathrm{ml} / \mathrm{min} . \quad 200 \mathrm{Bar}$

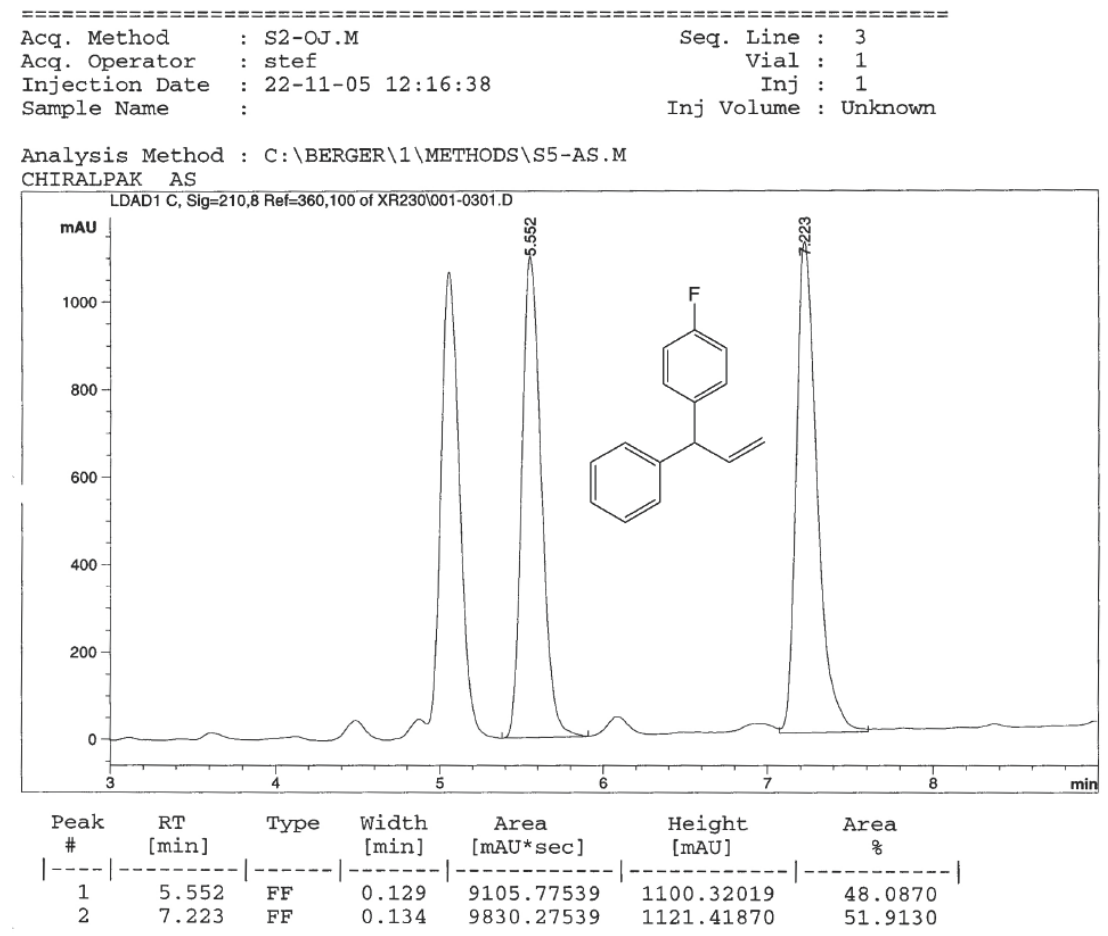

OJ-H 28-2-1-158 200 bars $2 \mathrm{ml} / \mathrm{min} \quad 30 \mathrm{C}$

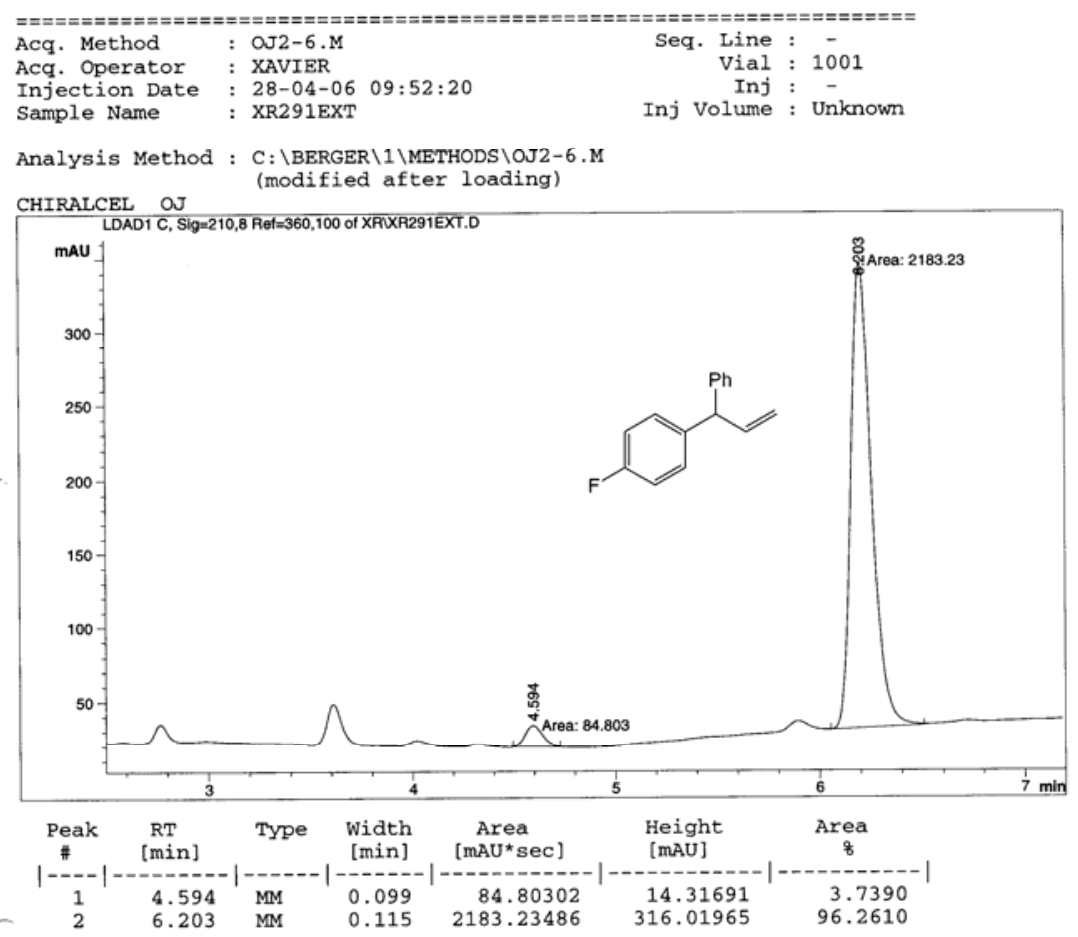




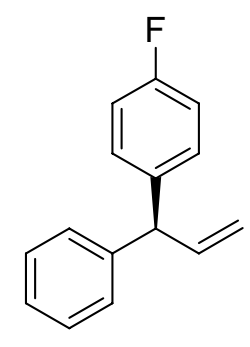

\section{1-(1-(4-fluorophenyl)allyl)benzene 14:}

The reaction proceeds on $0.5 \mathrm{mmol}$ scale of starting material 21 following GP/NS1 and using ligand L1 (S,S,S). The product was purified by flash column chromatography (silica, pentane), to afford $29.1 \mathrm{mg}$ ( $27 \%$ yield) of desired compound $\mathbf{1 4}$ as a slightly yellow liquid and $71.4 \mathrm{mg}$ (67\% yield) of SN2'-SN2 mixture. Ee of $82 \%$ was measured by chiral SFC with a OJ-H column (program: 2\% MeOH-2'-1-15\%, 200 bar, $\left.2 \mathrm{ml} / \mathrm{min}, 30^{\circ} \mathrm{C}\right) . \mathrm{R}_{\mathrm{T}}: 5.05(\mathrm{~S})$ and $6.74(\mathrm{R}) .[\alpha]_{\mathrm{D}}{ }^{20}=-2.9\left(\mathrm{c}=0.84, \mathrm{CHCl}_{3}\right){ }^{1} \mathrm{H} \mathrm{NMR}$ $\left(400 \mathrm{MHz}, \mathrm{CDCl}_{3}\right) \delta$ 6.97-7.53 (m, 9H), $6.29(\mathrm{~m}, 1 \mathrm{H}),, 5.25(\mathrm{~d}, 1 \mathrm{H}, \mathrm{J}=10.1 \mathrm{~Hz}), 4.99(\mathrm{~d}, 1 \mathrm{H}, \mathrm{J}=17.2 \mathrm{~Hz}), 4.73(\mathrm{~d}, 1 \mathrm{H}, \mathrm{J}=7.0 \mathrm{~Hz}) .{ }^{13} \mathrm{C} \mathrm{NMR}$ $\left(100 \mathrm{MHz} \mathrm{CDCl}_{3}\right) \delta 143.0,140.4,130.04,129.96,128.6,128.49,128.46$ (2C), 126.5, 116.5, 115.8, 115.5, 115.2, $115.1,54.1$.

og-H $\quad 28-2-1-15 \%-0 \quad 2 \mathrm{ml} / \mathrm{min} . \quad 200 \mathrm{Bar}$

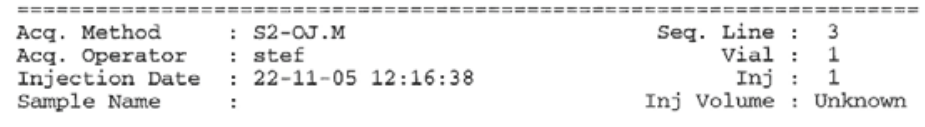

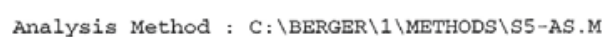

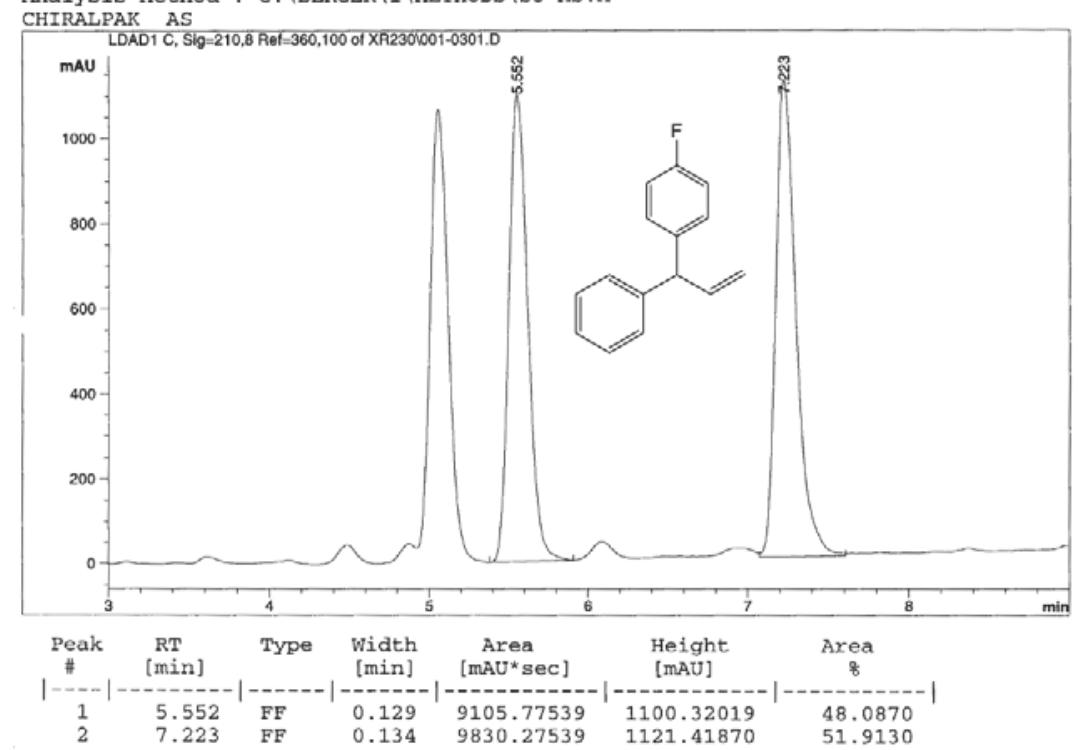

OJ-H $28-2-1-15 \% \quad 200$ bars $2 \mathrm{ml} / \mathrm{min} \quad 30 \mathrm{C}$

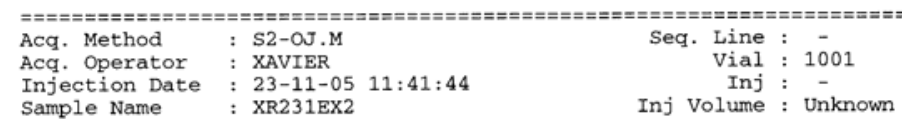

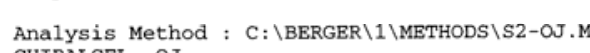

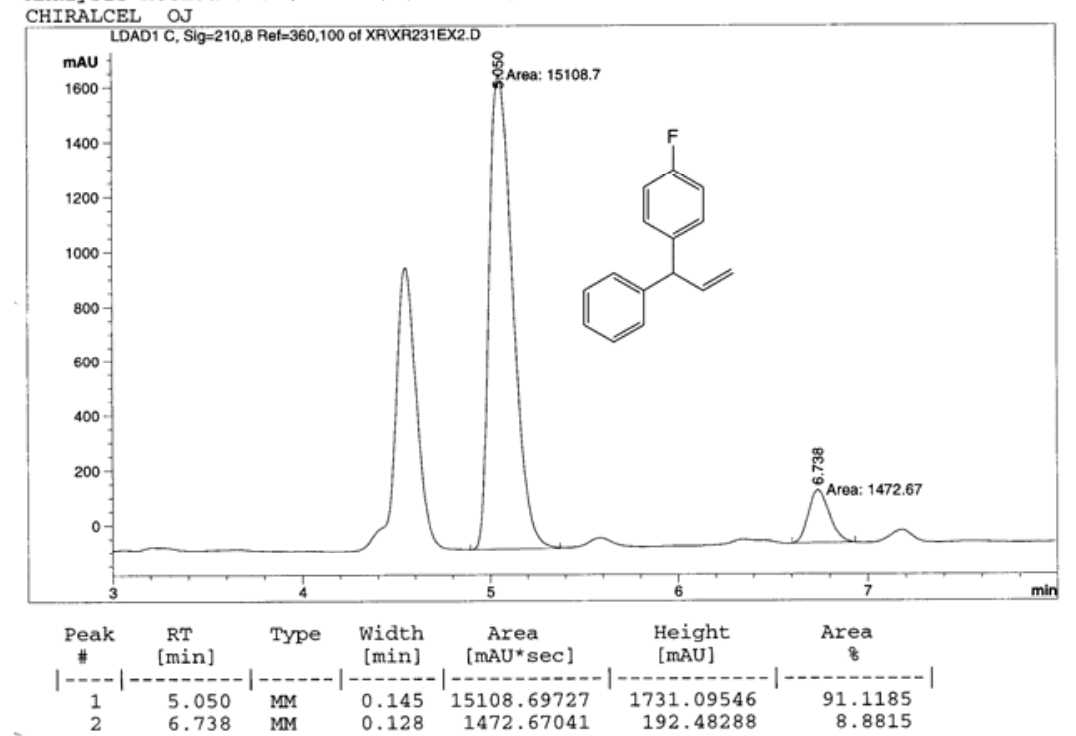


${ }^{1} \mathrm{H}-\mathrm{NMR}$

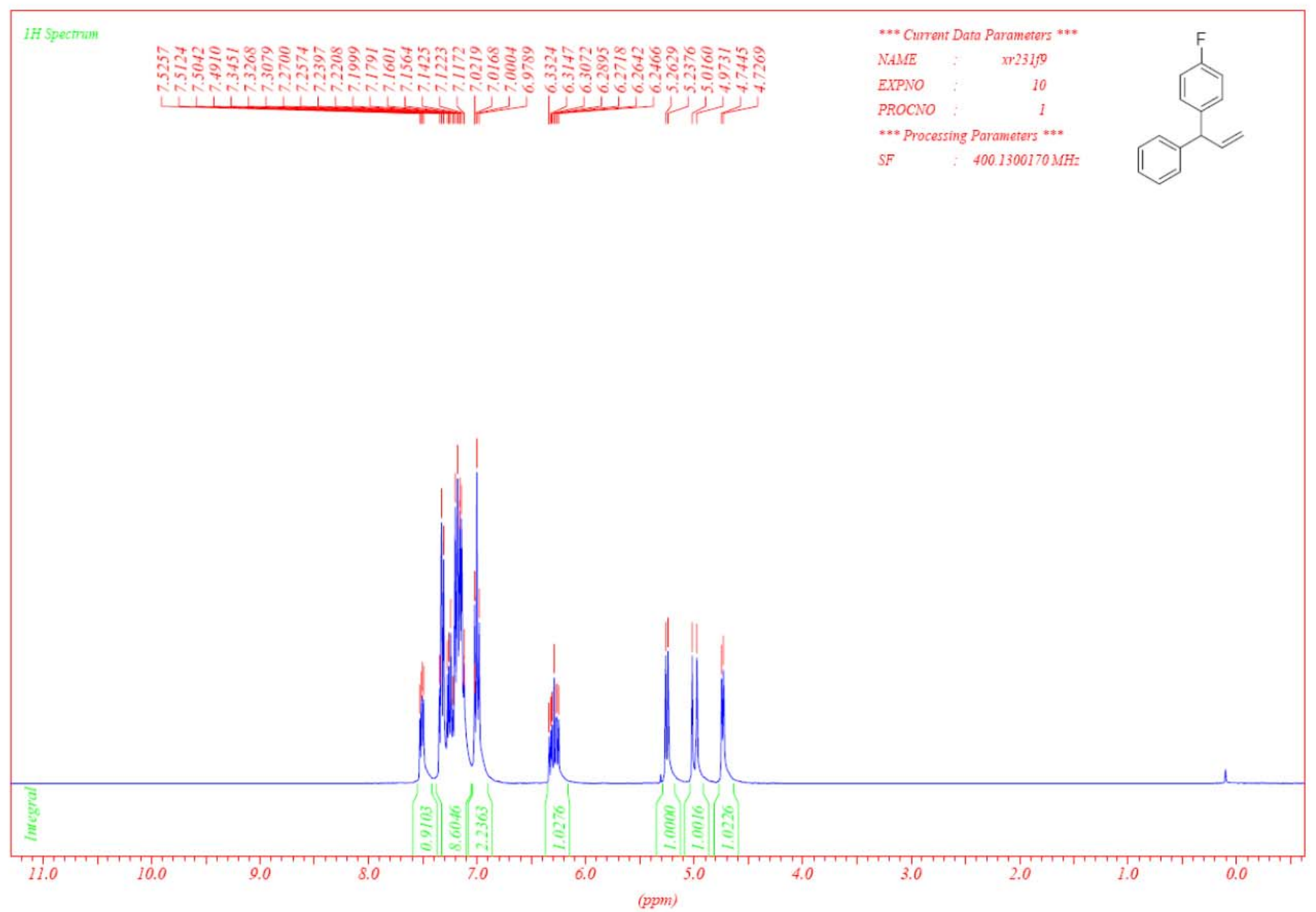




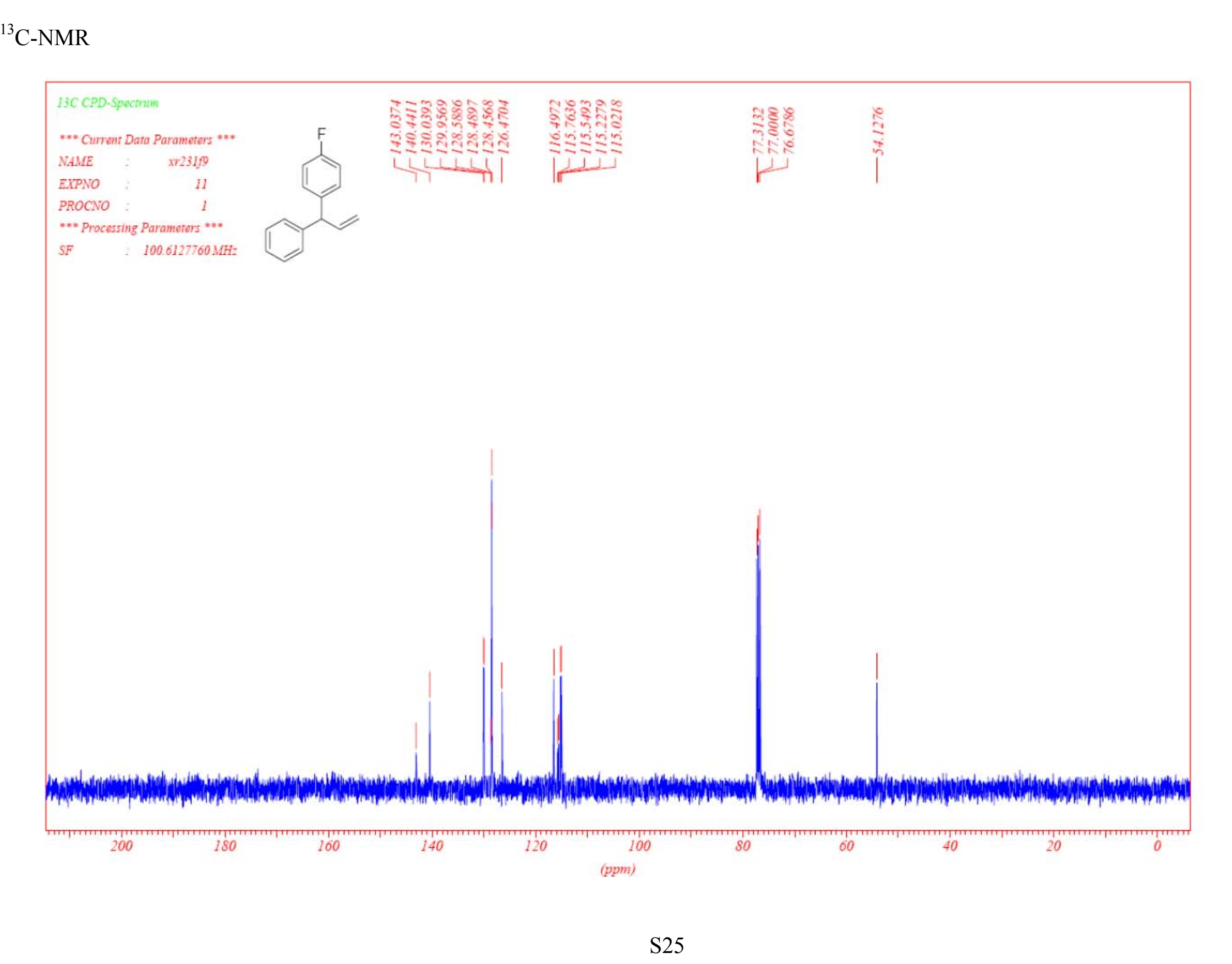




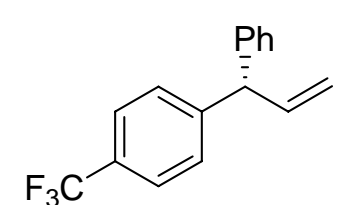

\section{1-(1-(4-(trifluoromethyl)phenyl)allyl)benzene 16:}

The reaction proceeds on $0.5 \mathrm{mmol}$ scale of starting material 15 following GP/NS1 and using ligand L1 (R,R,R). The product was purified by flash column chromatography (silica, pentane), to afford the SN2'-SN2 mixture (60/40) as a

colourless liquid (131.6 mg, 100\% yield). Ee of $97 \%$ was measured by chiral GC with a HYDRODEX TBDM column (program: $60^{\circ}-0^{\prime}-1^{\circ /}-$ $130^{\circ}-0^{\prime}-20^{\circ}-170^{\circ}-8^{\prime}$, speed $\left.49 \mathrm{~cm} / \mathrm{s}\right), \mathrm{R}_{\mathrm{T}}: 73.26(\mathrm{R})$ and $73.45(\mathrm{~S}) .{ }^{1} \mathrm{H}$ NMR $\left(400 \mathrm{MHz}, \mathrm{CDCl}_{3}\right) \delta$ 7.20-7.70 (m, $\left.9 \mathrm{H}\right), 6.35(\mathrm{~m}, 1 \mathrm{H}),, 5.34(\mathrm{~d}, 1 \mathrm{H}$, $\mathrm{J}=10.4 \mathrm{~Hz}), 5.07(\mathrm{~d}, 1 \mathrm{H}, \mathrm{J}=16.9 \mathrm{~Hz}), 4.84(\mathrm{~d}, 1 \mathrm{H}, \mathrm{J}=7.1 \mathrm{~Hz})$.
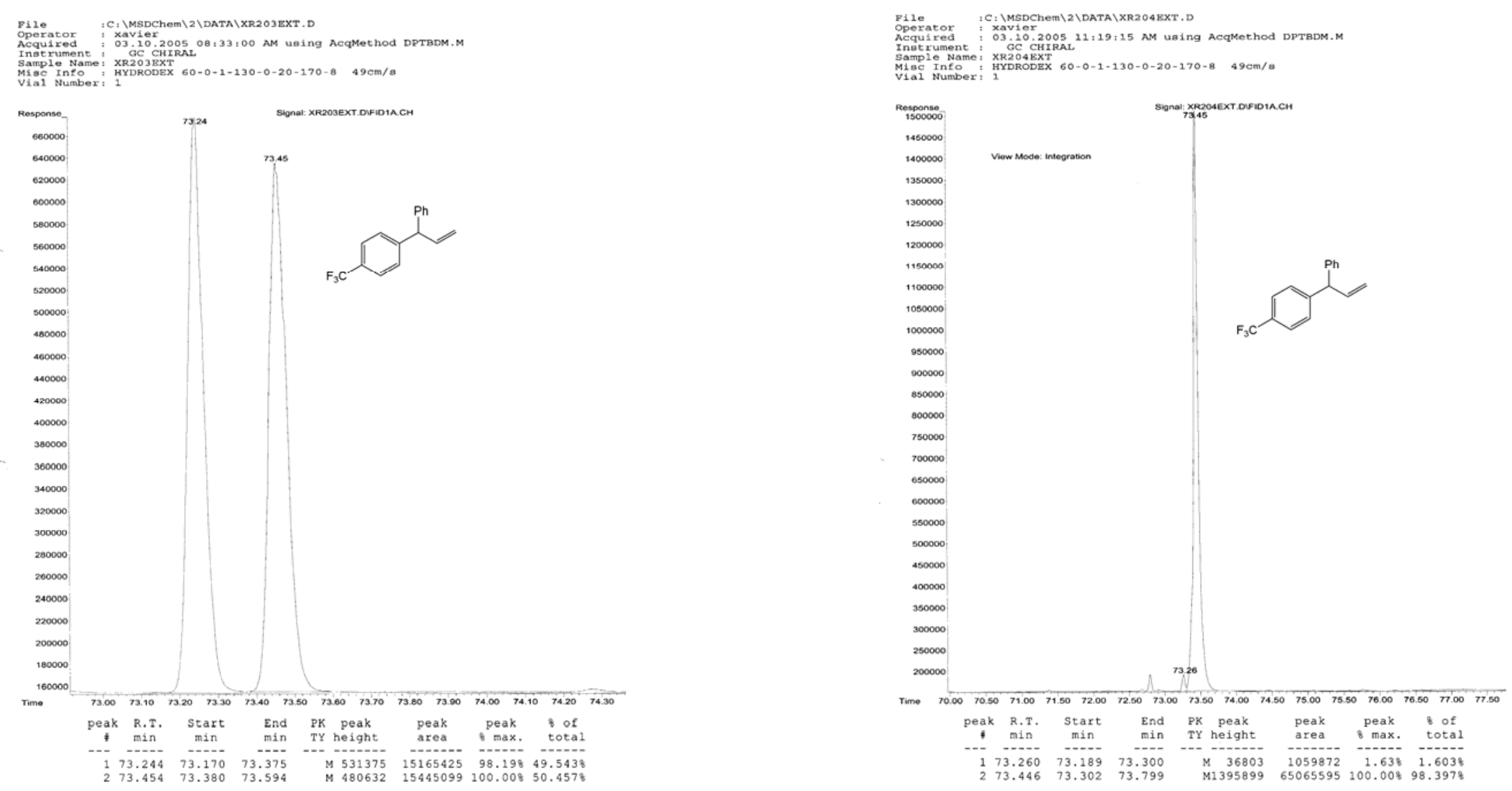
${ }^{1} \mathrm{H}-\mathrm{NMR}$

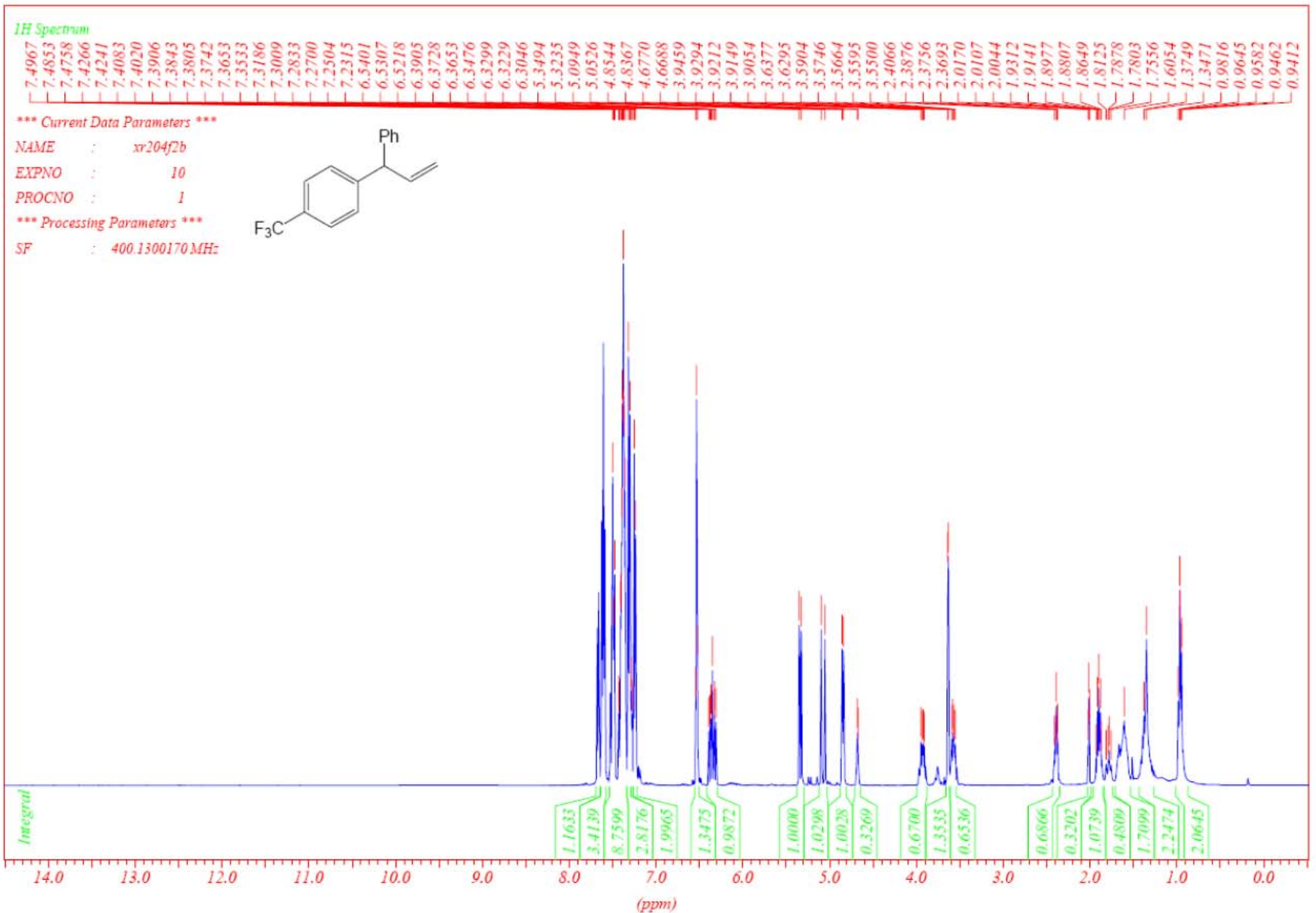




\section{${ }^{13} \mathrm{C}-\mathrm{NMR}$}

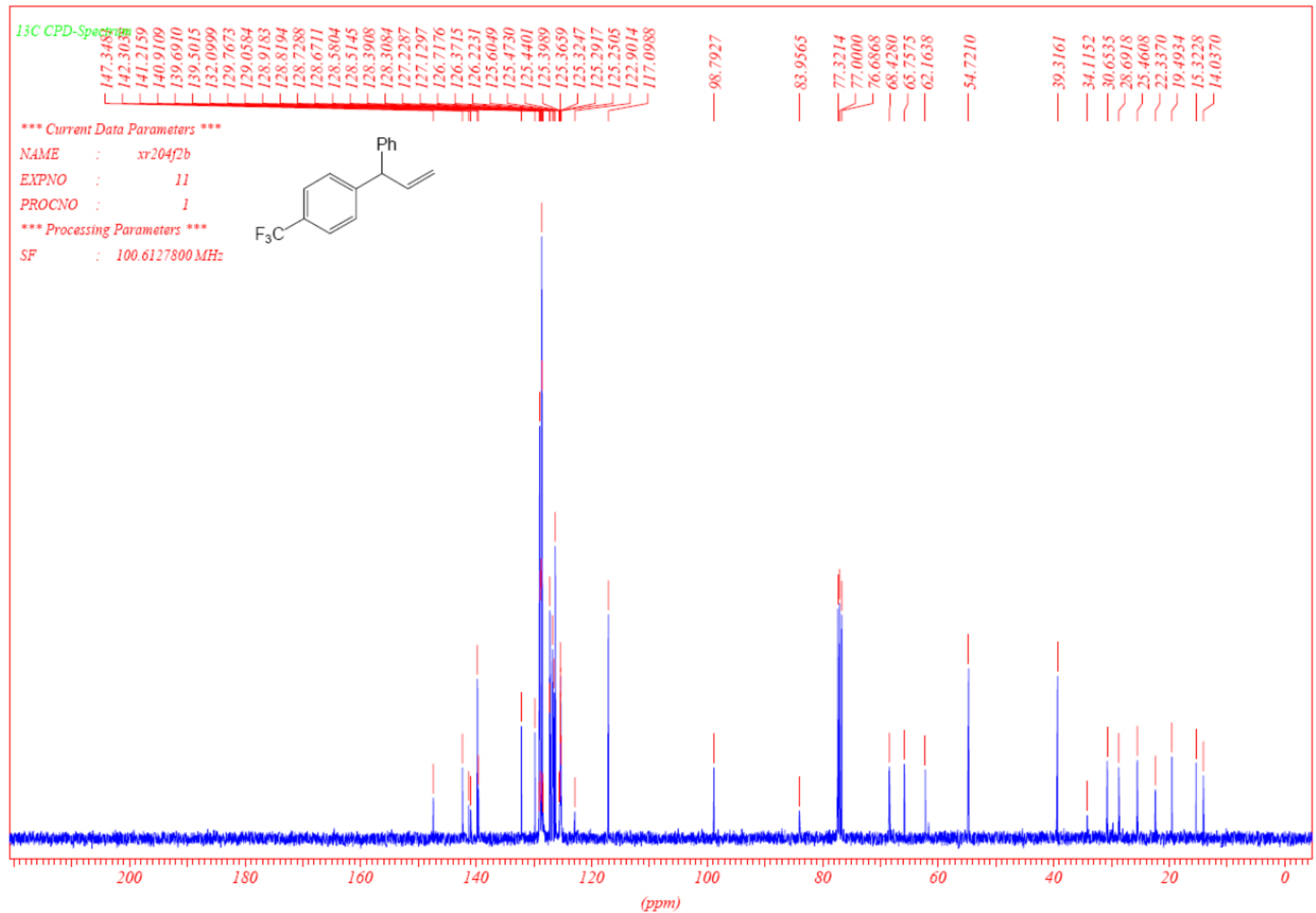




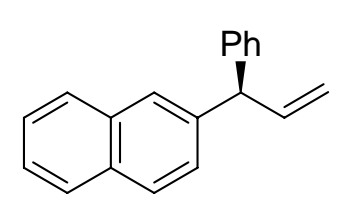

\section{2-((R)-1-phenylallyl)naphthalene 18:}

The reaction proceeds on $4.0 \mathrm{mmol}$ scale of starting material 17 following GP/NS1 and using ligand L1 (S,S,S). The product was purified by flash column chromatography (silica, pentane), to afford $386.9 \mathrm{mg}$ (40\% yield) of desired compound 18 as a colourless liquid and $520 \mathrm{mg}$ (53\% yield) of SN2'-SN2 mixture. Ee of $92 \%$ was measured by chiral SFC with a OJ-H column (program: 10\% MeOH-10'-1-15\%, 200 bar, $\left.2 \mathrm{ml} / \mathrm{min}, 30^{\circ} \mathrm{C}\right) . \mathrm{R}_{\mathrm{T}}$ : $12.24(\mathrm{~S})$ and $13.74(\mathrm{R}) .[\alpha]_{\mathrm{D}}{ }^{20}=-13.1(\mathrm{c}=1.03, \mathrm{CHCl}) .{ }^{1} \mathrm{H} \mathrm{NMR}(400 \mathrm{MHz}$, $\left.\mathrm{CDCl}_{3}\right) \delta$ 7.83-7.25 (m, 12H), $6.45(\mathrm{ddd}, 1 \mathrm{H}, \mathrm{J}=17.2 \mathrm{~Hz}, \mathrm{~J}=10.1 \mathrm{~Hz}, \mathrm{~J}=1.3 \mathrm{~Hz}), 5.33(\mathrm{dt}, 1 \mathrm{H}, \mathrm{J}=10.1 \mathrm{~Hz}, \mathrm{~J}=1.3 \mathrm{~Hz}), 5.10(\mathrm{dt}, 1 \mathrm{H}, \mathrm{J}=17.2$ $\mathrm{Hz}, \mathrm{J}=1.3 \mathrm{~Hz}), 4.95(\mathrm{~d}, 1 \mathrm{H}, \mathrm{J}=7.1 \mathrm{~Hz}) .{ }^{13} \mathrm{C} \mathrm{NMR}\left(100 \mathrm{MHz}, \mathrm{CDCl}_{3}\right) \delta 143.1,140.7,140.4,133.4,132.2,128.7$ (2C), $128.4(2 \mathrm{C}), 128.0,127.7$, $127.5,127.3,126.8,126.4,126.0,125.5,116.6,55.0$.

OJ $108-10-1-15 \quad 2 \mathrm{ml} / \mathrm{mn} 200$ Bar $30 \mathrm{C}$

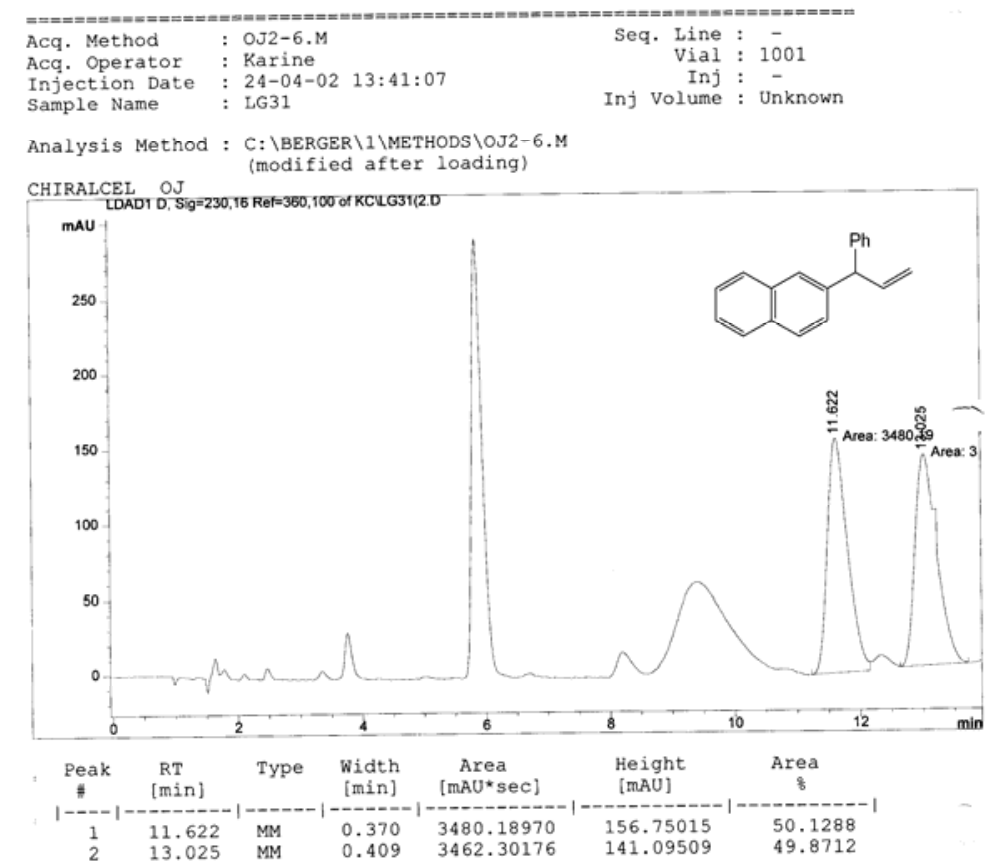

OU-H $\quad 108-10-1-158 \quad 200$ bars $2 \mathrm{ml} / \mathrm{min} \quad 30 \mathrm{C}$

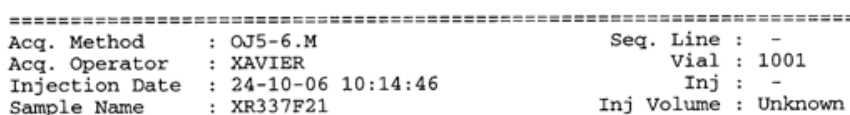

Analysis Method : $C: \backslash$ BERGER $\backslash 1 \backslash M E T H O D S \backslash$ OJ5 $5-6 . M$

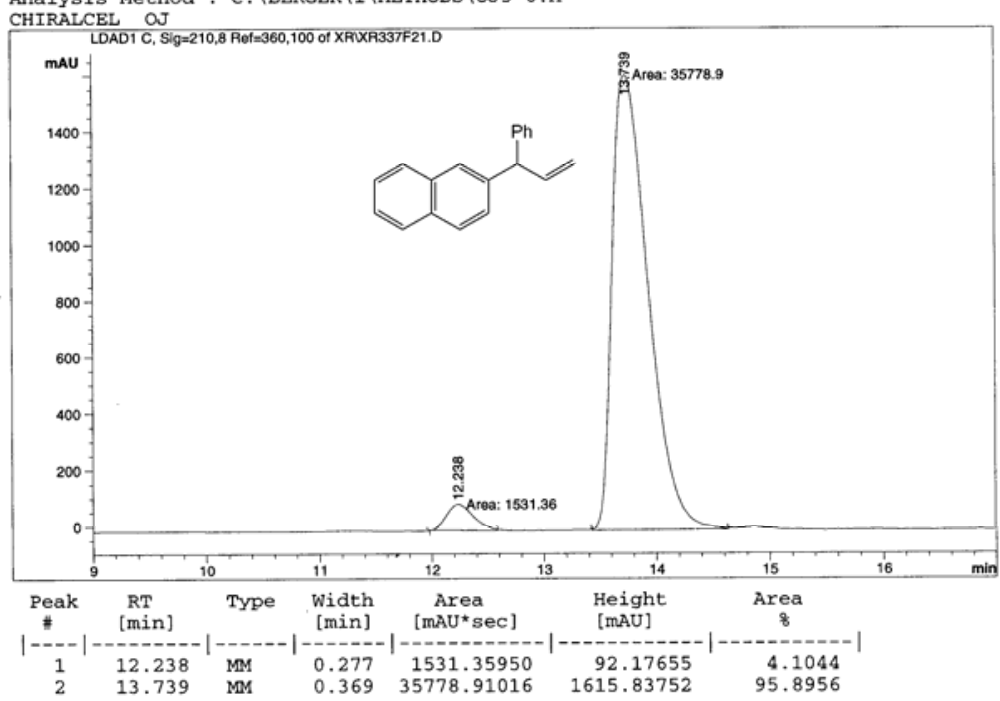




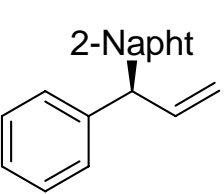

\section{2-((S)-1-phenylallyl)naphthalene 18:}

The reaction proceeds on $0.5 \mathrm{mmol}$ scale of starting material 21 following GP/NS2 and using ligand L1 (S,S,S). The product was purified by flash column chromatography (silica, pentane), to afford the SN2'-SN2 mixture (38/62) as a colourless liquid (94.7

$\mathrm{mg}, 78 \%$ yield). Ee of $80 \%$ was measured by chiral SFC with a OJ-H column (program: $10 \% \mathrm{MeOH}-10^{\prime}-1-15 \%, 200 \mathrm{bar}, 2 \mathrm{ml} / \mathrm{min}, 30{ }^{\circ} \mathrm{C}$ ). $\mathrm{R}_{\mathrm{T}}$ : $13.00(\mathrm{~S})$ and 14.77(R). ${ }^{1} \mathrm{H} \mathrm{NMR}\left(400 \mathrm{MHz}, \mathrm{CDCl}_{3}\right) \delta$ 7.83-7.25 (m, 12H), $6.45(\mathrm{ddd}, 1 \mathrm{H}, \mathrm{J}=17.2 \mathrm{~Hz}, \mathrm{~J}=10.1 \mathrm{~Hz}, \mathrm{~J}=1.3 \mathrm{~Hz}), 5.33(\mathrm{dt}, 1 \mathrm{H}, \mathrm{J}=$ $10.1 \mathrm{~Hz}, \mathrm{~J}=1.3 \mathrm{~Hz}), 5.10(\mathrm{dt}, 1 \mathrm{H}, \mathrm{J}=17.2 \mathrm{~Hz}, \mathrm{~J}=1.3 \mathrm{~Hz}), 4.95(\mathrm{~d}, 1 \mathrm{H}, \mathrm{J}=7.1 \mathrm{~Hz}) .{ }^{13} \mathrm{C} \mathrm{NMR}\left(100 \mathrm{MHz}, \mathrm{CDCl}_{3}\right) \delta 143.1,140.7,140.4,133.4$, $132.2,128.7(2 \mathrm{C}), 128.4(2 \mathrm{C}), 128.0,127.7,127.5,127.3,126.8,126.4,126.0,125.5,116.6,55.0$.

OJ $108-10-1-15 \quad 2 \mathrm{ml} / \mathrm{mn} 200$ Bar $30 \mathrm{C}$

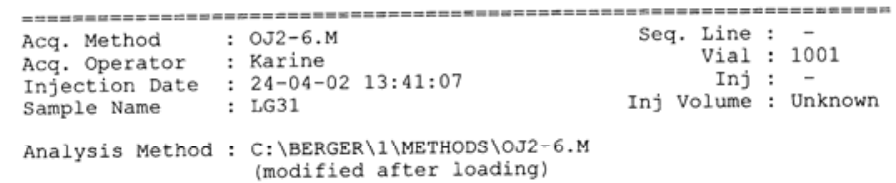

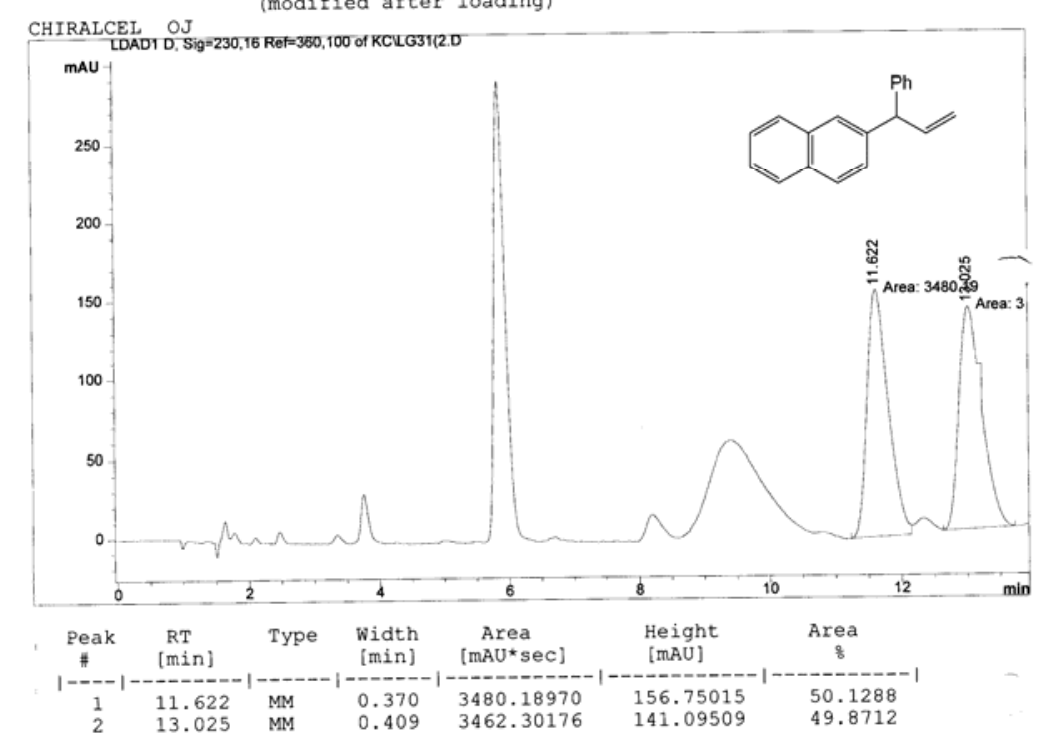

OT-H 108-10-1-15\% 200 bars $2 \mathrm{ml} / \mathrm{min} \quad 30 \mathrm{C}$

\begin{tabular}{|c|c|c|}
\hline $\begin{array}{l}\text { Acq. Method } \\
\text { Acq. Operator } \\
\text { Injection Date } \\
\text { Sample Name }\end{array}$ & $\begin{array}{l}: \text { OJ5-6.M } \\
: \text { XAVIER } \\
: 17-03-06 \quad 14: 57: 55 \\
: \text { XR272f3 }\end{array}$ & 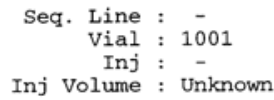 \\
\hline
\end{tabular}

Analysis Method : C: \BERGER \1\METHODS\OT5-6.M

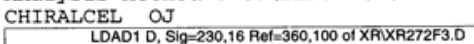

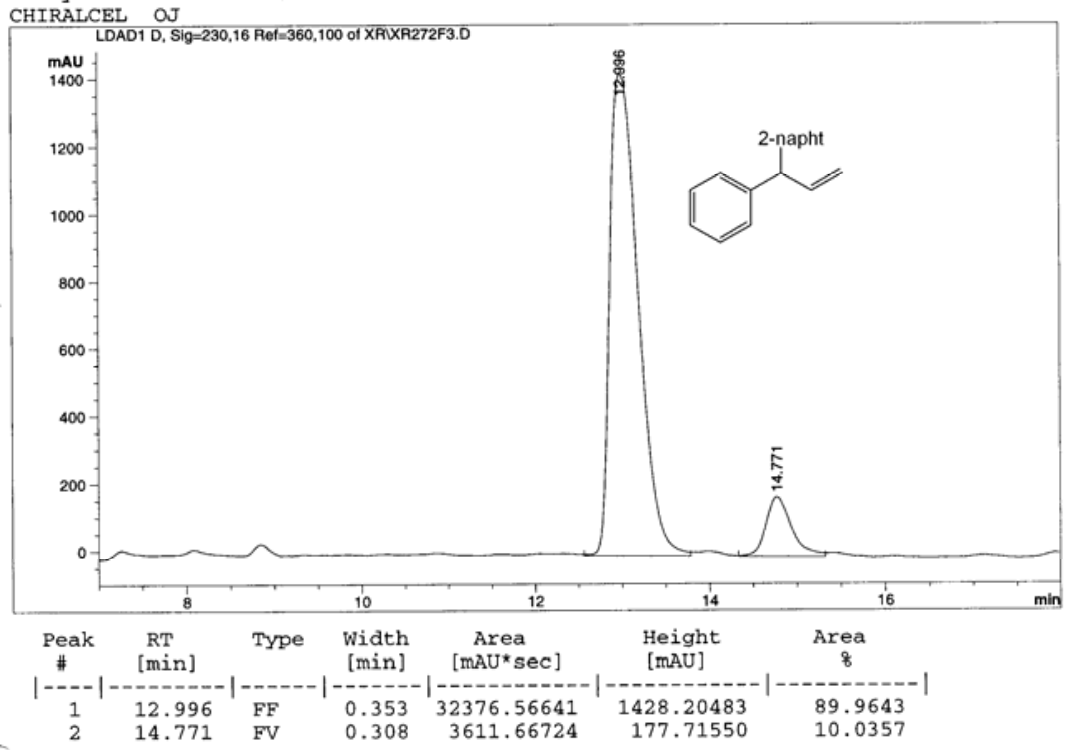


${ }^{1} \mathrm{H}-\mathrm{NMR}$

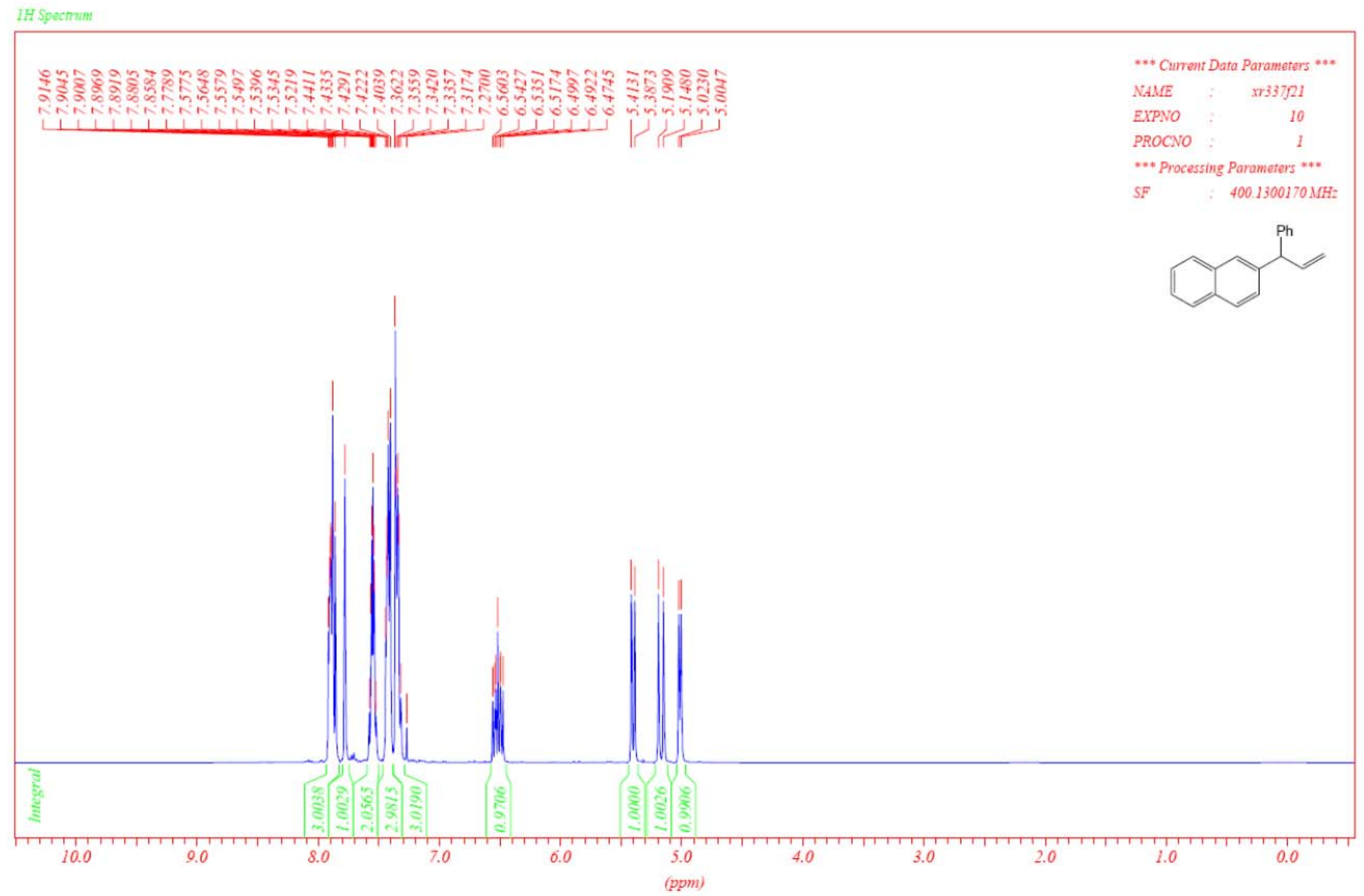


${ }^{13} \mathrm{C}-\mathrm{NMR}$

13C CPD-Spectrim

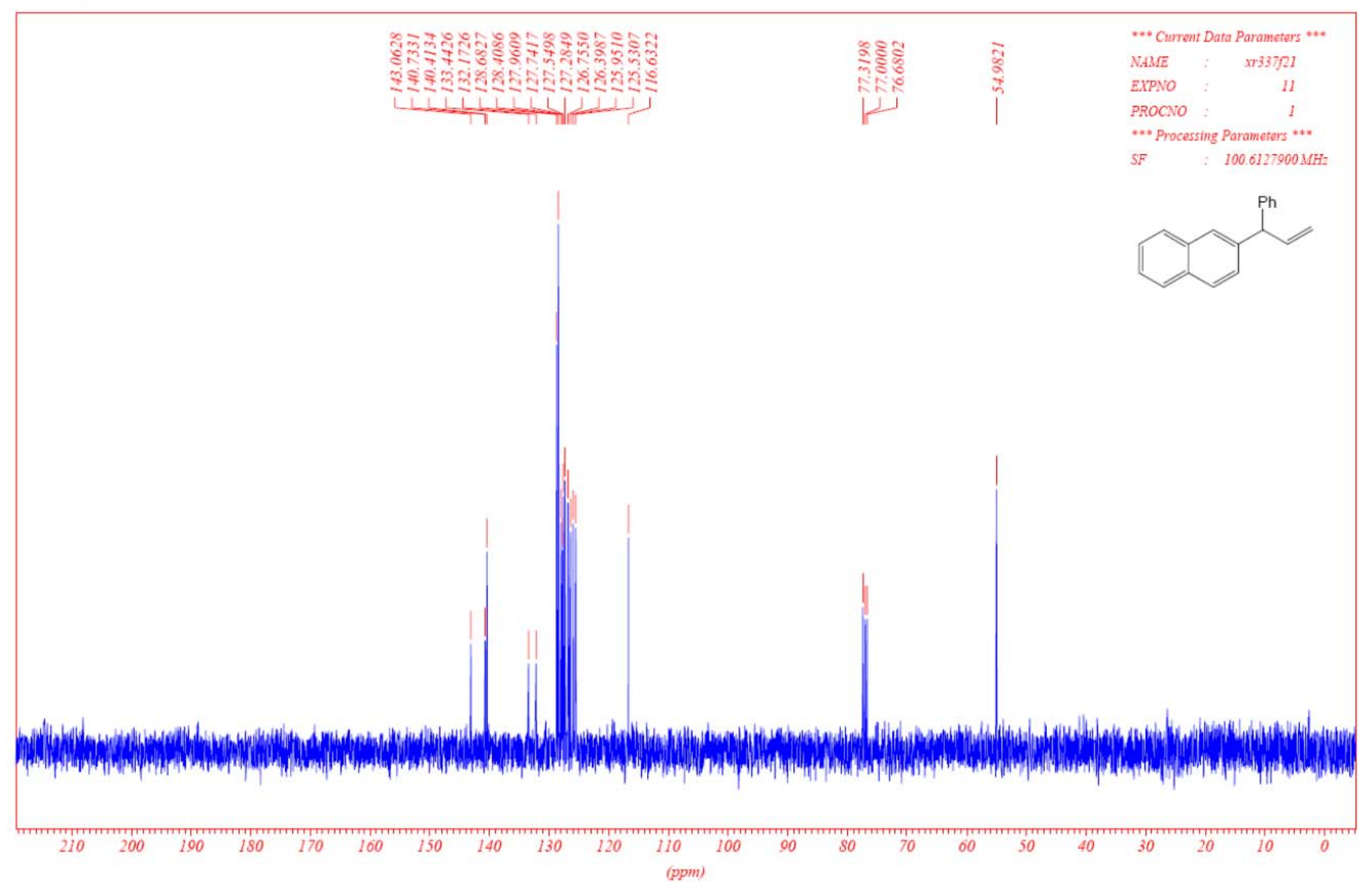




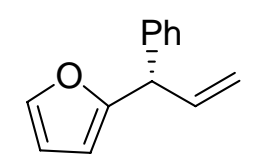

\section{2-((S)-1-phenylallyl)furan 20:}

The reaction proceeds on $0.5 \mathrm{mmol}$ scale of starting material 19 following GP/NS1 and using ligand L1 (S,S,S). The product was purified by flash column chromatography (silica, pentane), to afford the SN2'-SN2 mixture as a orange liquid (n.d. yield). Ee of

$79 \%$ was measured by chiral GC with a HYDRODEX TBDM column (program: 60- $0^{\prime}-1^{\circ}{ }^{\prime}-130^{\circ}-00^{\prime}-20^{\circ /}-170^{\circ}-8^{\prime}$, speed $\left.49 \mathrm{~cm} / \mathrm{s}\right), \mathrm{R}_{\mathrm{T}}: 52.54(\mathrm{~S})$ and $54.44(\mathrm{R}) .{ }^{1} \mathrm{H}$ NMR $\left(400 \mathrm{MHz}, \mathrm{CDCl}_{3}\right) \delta$ 6.00-7.70 (m, 9H), $5.25(\mathrm{dt}, 1 \mathrm{H}, \mathrm{J}=10.1 \mathrm{~Hz}, \mathrm{~J}=1.2 \mathrm{~Hz}), 5.09(\mathrm{dt}, 1 \mathrm{H}, \mathrm{J}=17.1 \mathrm{~Hz}, \mathrm{~J}=1.5 \mathrm{~Hz})$, $4.79(\mathrm{~d}, 1 \mathrm{H}, \mathrm{J}=7.1 \mathrm{~Hz})$.
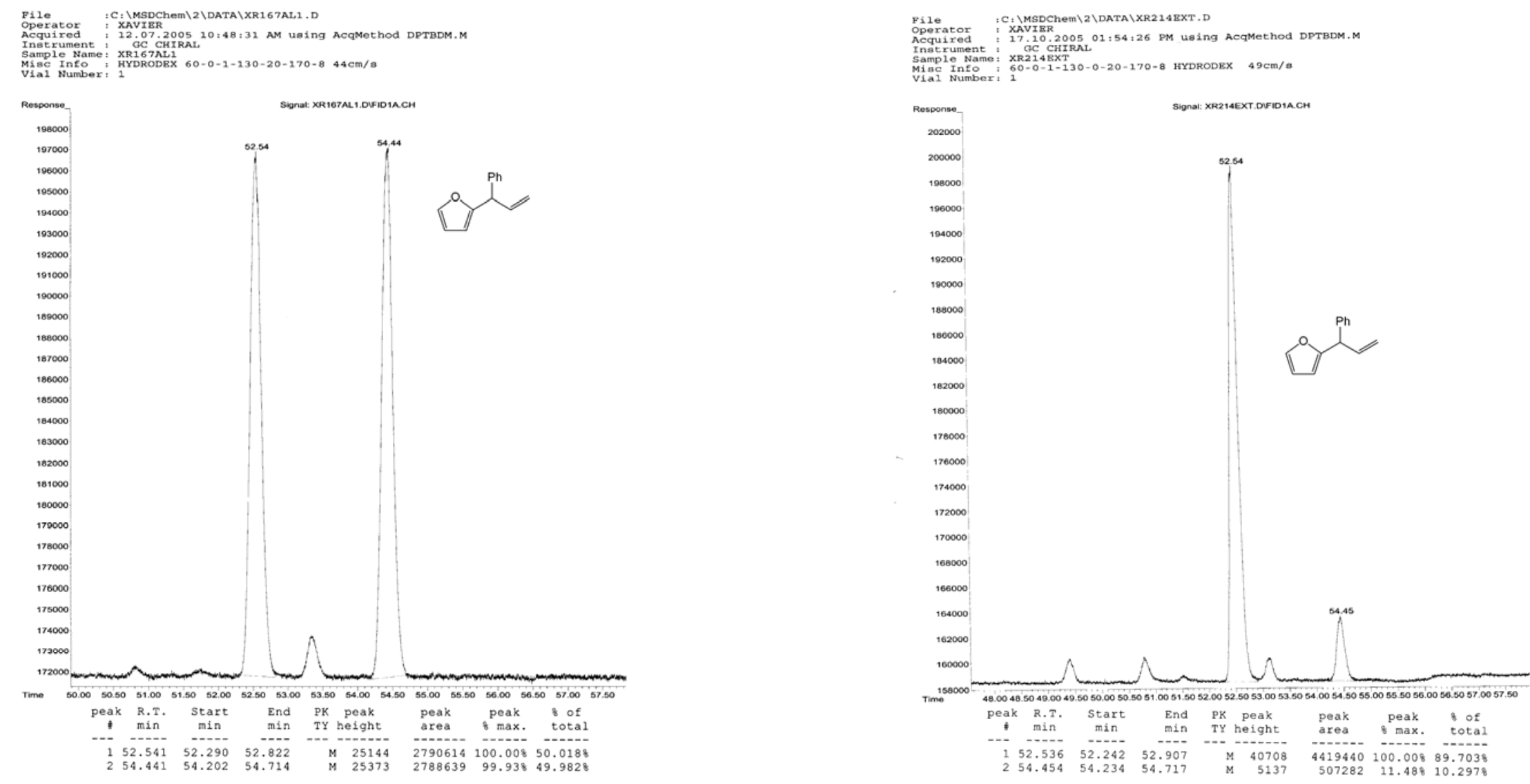


\section{${ }^{1} \mathrm{H}-\mathrm{NMR}$}

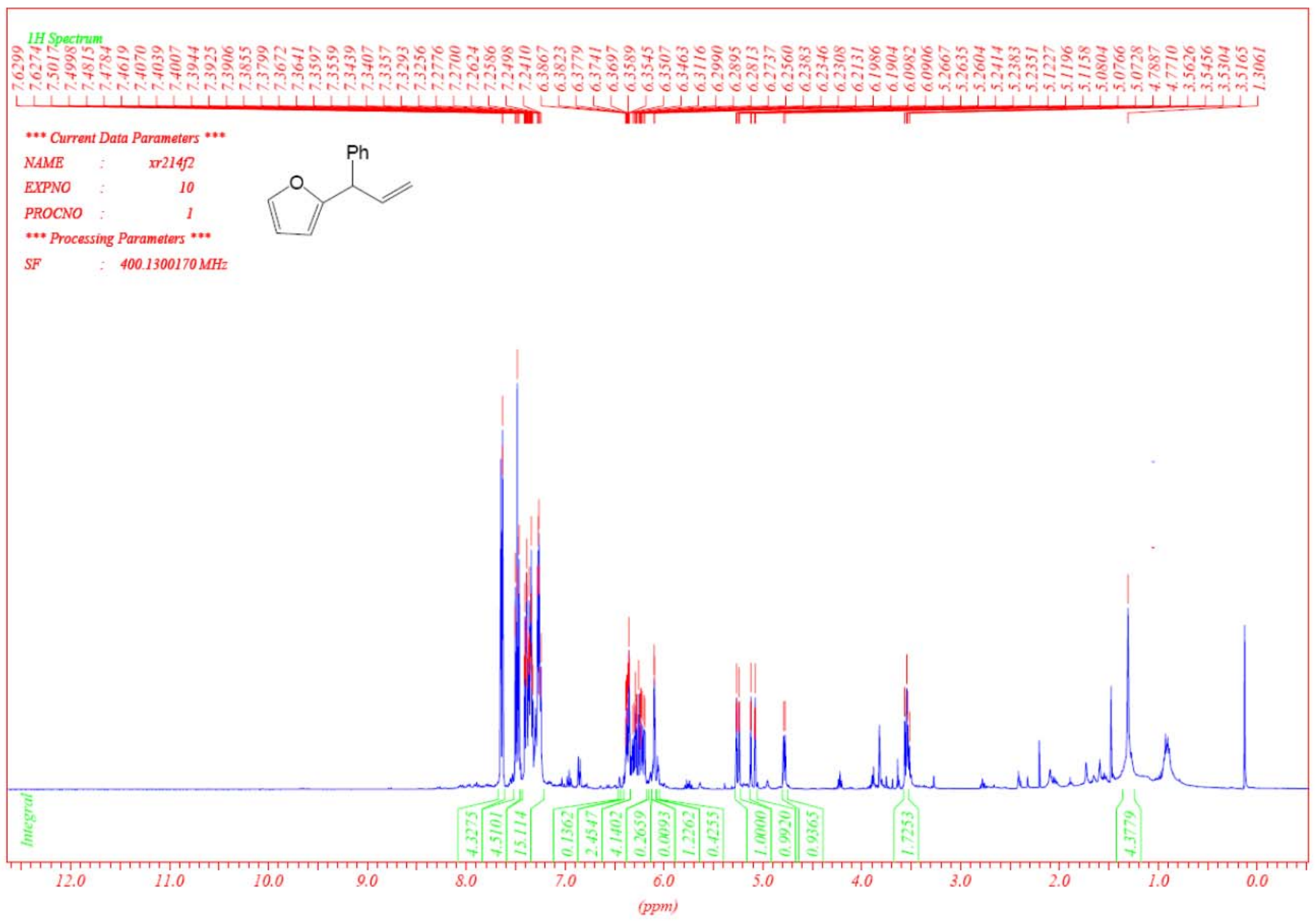


${ }^{13}$ C-NMR

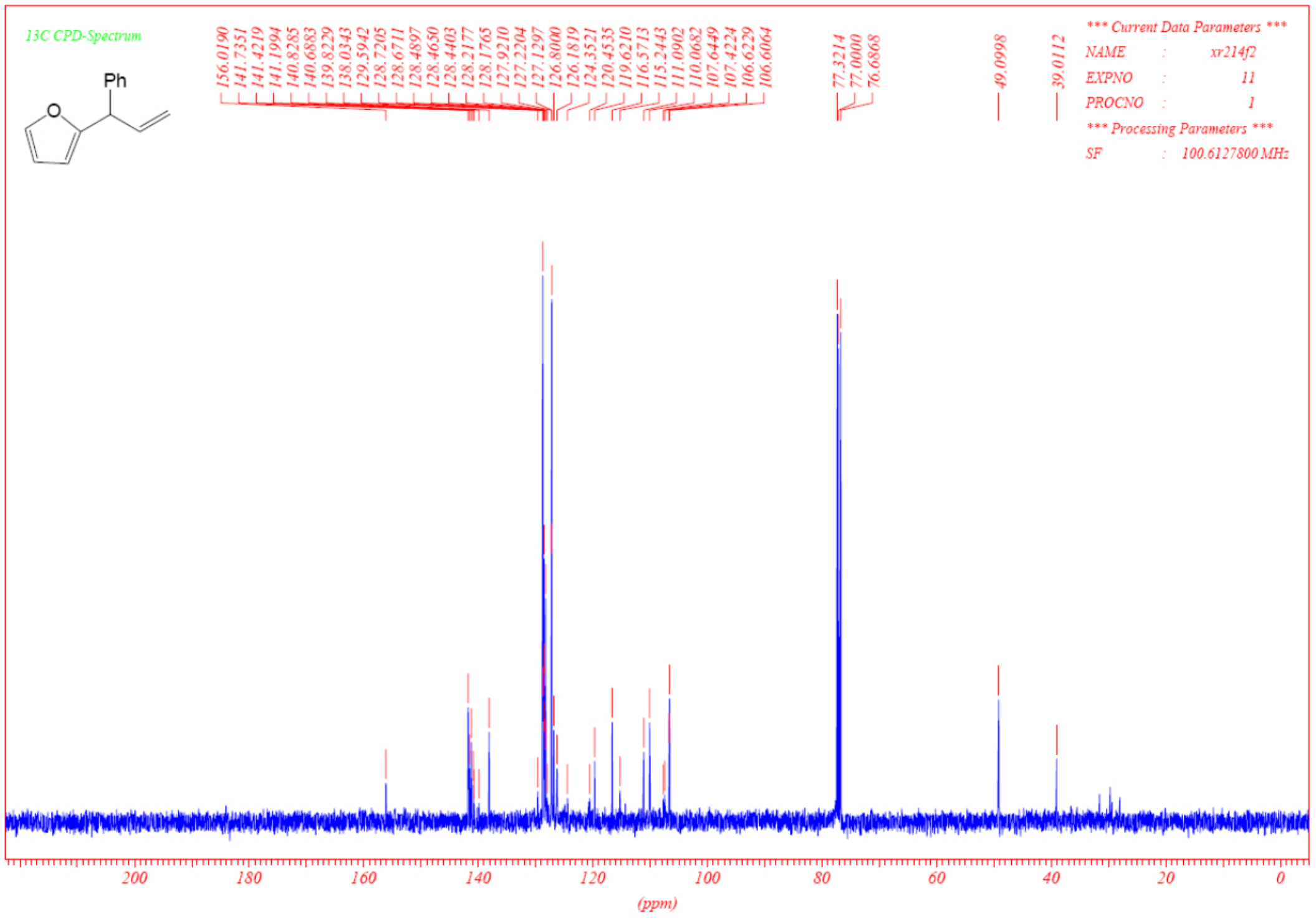




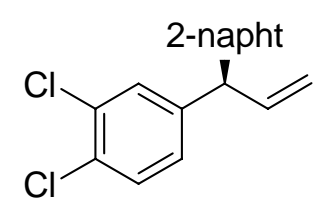

\section{2-((R)-1-(3,4-dichlorophenyl)allyl)naphthalene 22:}

The reaction proceeds on $2.0 \mathrm{mmol}$ scale of starting material 11 following GP/NS2 and using ligand L1 (S,S,S). The product was purified by flash column chromatography (silica, pentane), $81.4 \mathrm{mg}$ (13\% yield) of desired compound 22 as a colourless oil and $238.8 \mathrm{mg}$ (38\% yield) of SN2'-SN2 mixture. Ee of $86 \%$ was measured by chiral SFC with a OD-H column (program: 2\% MeOH-2'-1$15 \%, 200$ bar, $\left.2 \mathrm{ml} / \mathrm{min}, 30^{\circ} \mathrm{C}\right) . \mathrm{R}_{\mathrm{T}}: 5.68(\mathrm{R})$ and $5.89(\mathrm{~S}) .[\alpha]_{\mathrm{D}}{ }^{20}=+18.6\left(\mathrm{c}=1.04, \mathrm{CHCl}_{3}\right) .{ }^{1} \mathrm{H} \mathrm{NMR}(400 \mathrm{MHz}, \mathrm{CDCl} 3) \delta 7.83(\mathrm{~m}, 3 \mathrm{H}), 7.65$ (s, 1H), $7.49(\mathrm{~m}, 2 \mathrm{H}), 7.39$ (d, 1H, J = 8.1 Hz), 7.35 (s, 1H), 7.28 (d, 1H, J = 8.4 Hz), 7.07 (d, 1H, J = 8.1 Hz), 6.35 (m, 1H), 5.35 (d, $1 \mathrm{H}, \mathrm{J}=10.4$ $\mathrm{Hz}), 5.08(\mathrm{~d}, 1 \mathrm{H}, \mathrm{J}=16.9 \mathrm{~Hz}), 4.87(\mathrm{~d}, 1 \mathrm{H}, \mathrm{J}=7.1 \mathrm{~Hz}) .{ }^{13} \mathrm{C} \mathrm{NMR}\left(100 \mathrm{MHz}, \mathrm{CDCl}_{3}\right) \delta 143.4,139.5,133.4,132.4,132.3,130.6,130.5,130.3$, $128.3,128.2,127.8,127.6,126.9,126.2,125.9,117.6,54.1$.

OD-H $\quad 28-2-1-15 z-0 \quad 2 \mathrm{ml} / \mathrm{min} . \quad 200 \mathrm{Bar}$

\begin{tabular}{|c|c|c|}
\hline $\begin{array}{l}\text { Acq. Method } \\
\text { Acq. Operator } \\
\text { Injection Date } \\
\text { Sample Name }\end{array}$ & $\begin{array}{l}\text { : S2-OD.M } \\
: \begin{array}{l}\text { stef } \\
07-11-06 \quad 12: 40: 48\end{array}\end{array}$ & $\begin{array}{r:c}\text { Seq. Line : } & 1 \\
\text { Vial } & 1 \\
\text { Inj } & 1 \\
\text { Inj Volume } & \text { Unknown }\end{array}$ \\
\hline
\end{tabular}

Analysis Method : $C: \backslash$ BERGER $\backslash 1 \backslash M E T H O D S \backslash S 2-A D . M$
CHIRALCEL AD

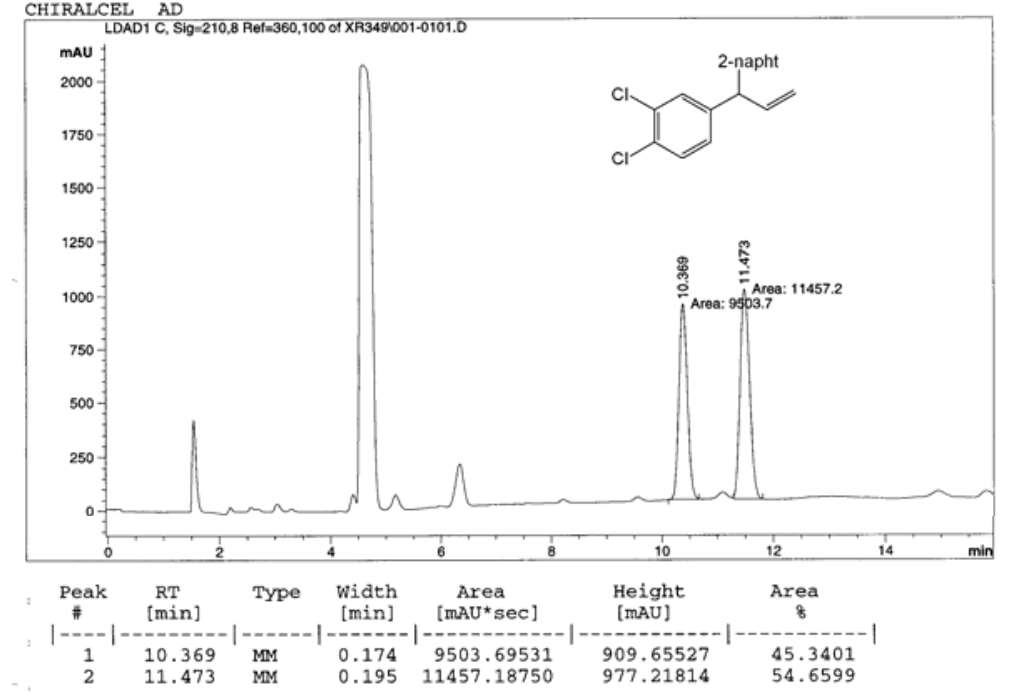

OD-H $\quad 28-2-1-15 \% \quad 200$ bars $2 \mathrm{ml} / \mathrm{min} \quad 30 \mathrm{C}$

\begin{tabular}{|c|c|c|}
\hline $\begin{array}{l}\text { Acq. Method } \\
\text { Acq. Operator } \\
\text { Injection Date } \\
\text { Sample Name }\end{array}$ & $\begin{array}{l}\text { OD-H2-6.M } \\
\text { XAVIRR } \\
: 10-11-06 \quad 09: 17: 28 \\
\text { XR352F68 }\end{array}$ & $\begin{array}{r:l}\text { Seq. Line } & - \\
\text { Vial } & 1001 \\
\text { Inj } & - \\
\text { Inj Volume } & \text { Unknown }\end{array}$ \\
\hline
\end{tabular}

Analysis Method : C: \BERGER \1\METHODS\OJ2-6.M

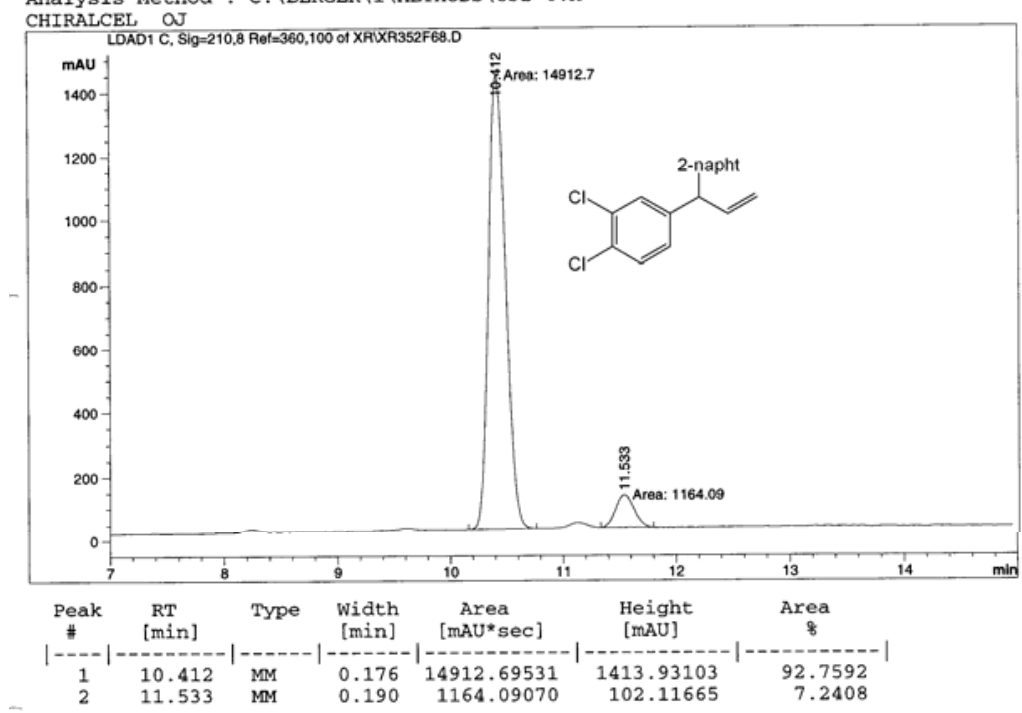




\section{${ }^{1} \mathrm{H}-\mathrm{NMR}$}

iH spectum

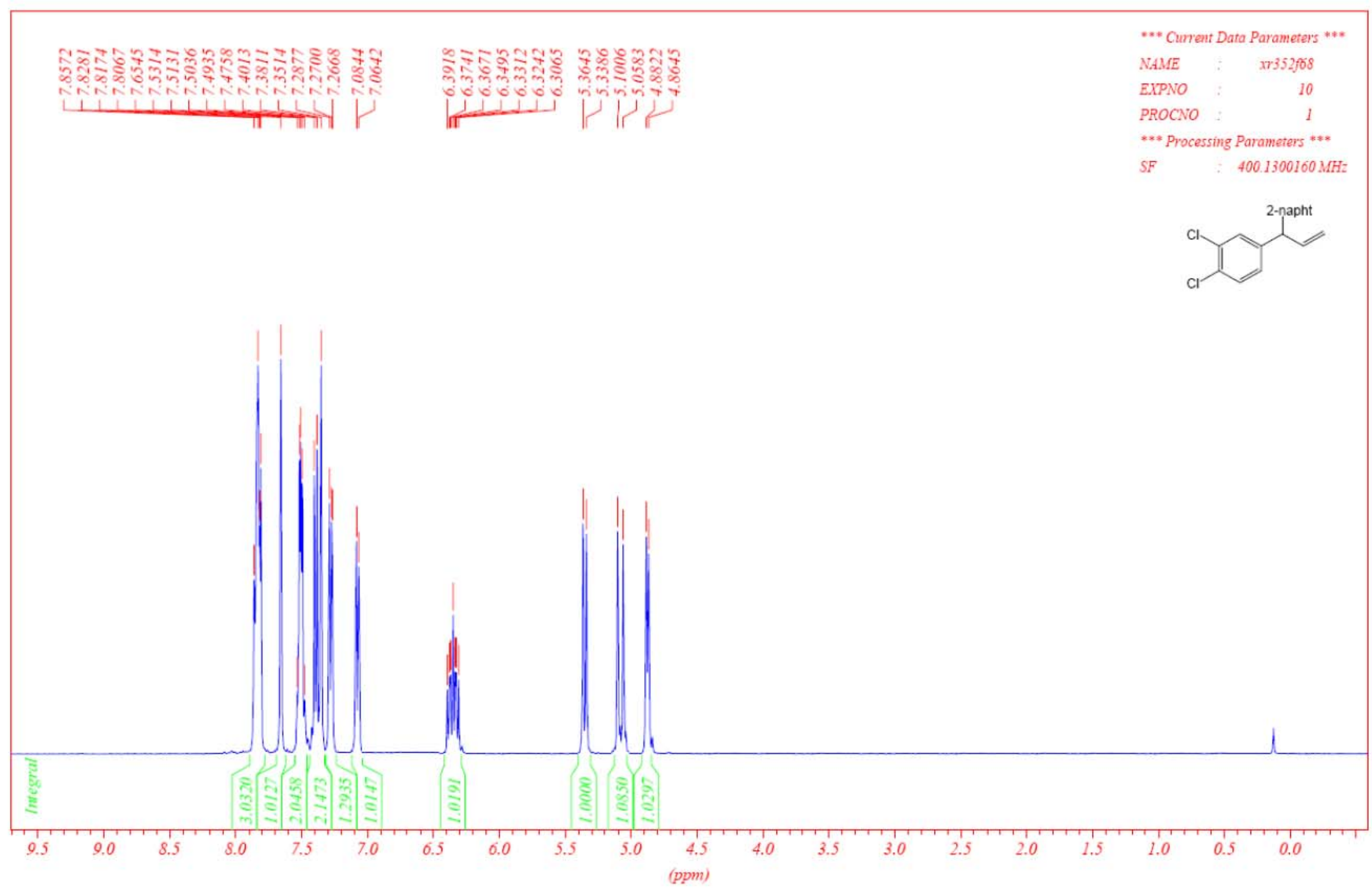


${ }^{13} \mathrm{C}-\mathrm{NMR}$

$13 C$ CPD-5pectrom

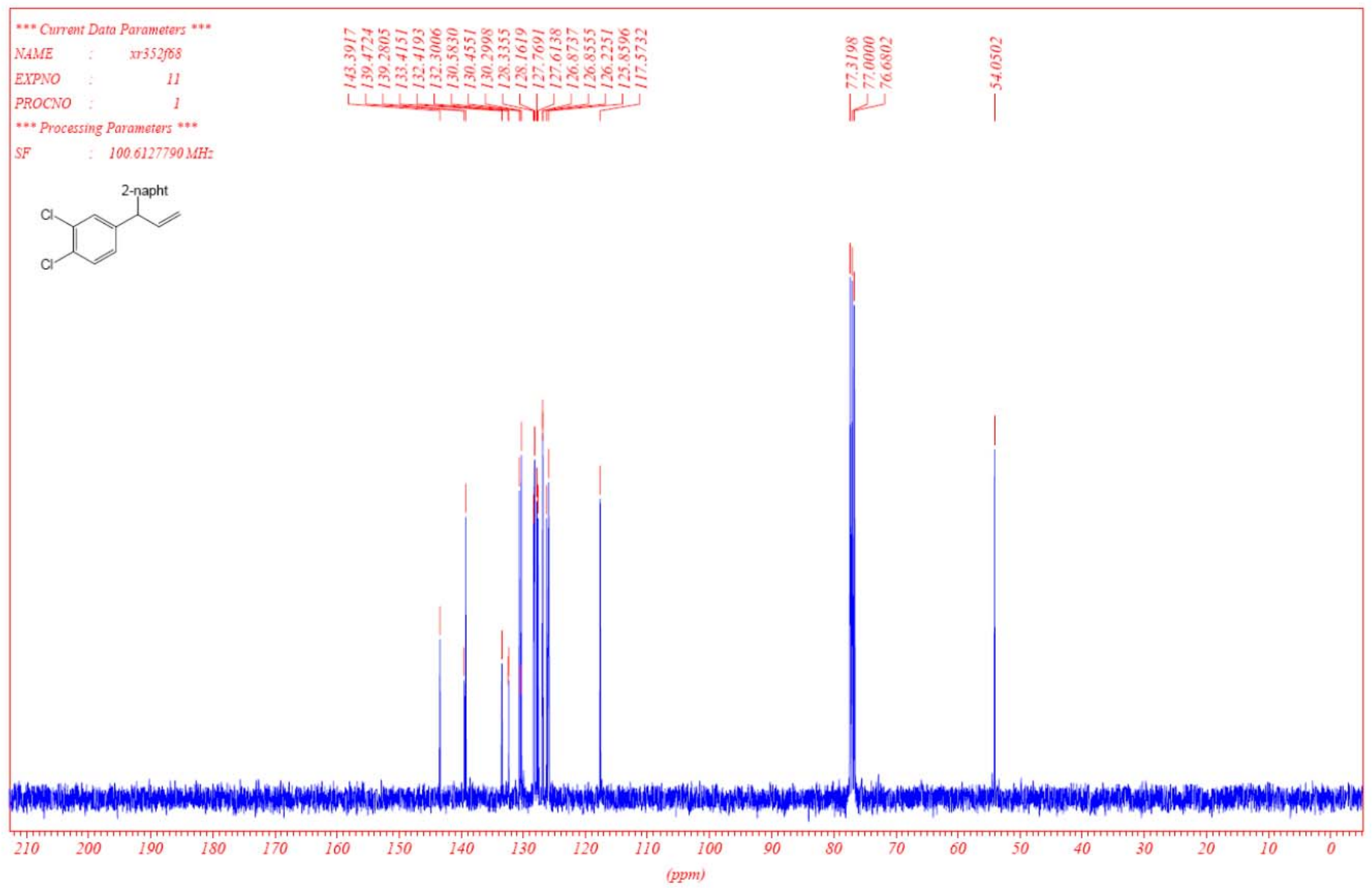

S38 


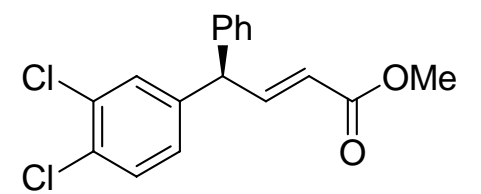

\section{(R)-methyl 4-(3,4-dichlorophenyl)-4-phenylbut-2-enoate 23:}

1,2-dichloro-4-((R)-1-phenylallyl)benzene $12(551.2 \mathrm{mg}, 2.1 \mathrm{mmol}, 1$ eq.) was added under argon into the flask containing $3 \mathrm{ml}$ of $\mathrm{CH}_{2} \mathrm{Cl}_{2}$, following by methylacrylate $\left(0.43 \mathrm{ml}, 4.75 \mathrm{mmol}, 2.3\right.$ éq.) and Hoveyda-Grubbs $2^{\text {nd }}$ generation catalyst $(29.8 \mathrm{mg}, 2.3 \%)$. The mixture was put under reflux overnight. The mixture was diluted with $\mathrm{Et}_{2} \mathrm{O}$ and then hydrolysed with $\mathrm{HCl} 10 \%$. The organic layer was extracted and the aqueous layer was washed 3 times with $\mathrm{Et}_{2} \mathrm{O}$. The combined organic layers were washed once with $\mathrm{NaCl}$ sat. and then dried with anhydrous $\mathrm{Na}_{2} \mathrm{SO}_{4}$, filtered and concentrated in vacuo. The product was purified by flash column chromatography (silica, pentane/ $\mathrm{Et}_{2} \mathrm{O} 9 / 1$ ), to afford desired compound as a colourless oil (568.1 mg, 84\% yield). $\left.{ }^{1} \mathrm{H} \mathrm{NMR} \mathrm{(400} \mathrm{MHz}, \mathrm{CDCl}_{3}\right) \delta$ 7.40-7.20 (m, 6H), $7.15(\mathrm{~d}, 2 \mathrm{H}, \mathrm{J}=7.8 \mathrm{~Hz}), 7.01(\mathrm{~d}, 1 \mathrm{H}, \mathrm{J}=8.1 \mathrm{~Hz}), 5.76(\mathrm{~d}, 1 \mathrm{H}, \mathrm{J}=15.6 \mathrm{~Hz}), 4.84(\mathrm{~d}, 1 \mathrm{H}, \mathrm{J}=7.3 \mathrm{~Hz}) .{ }^{13} \mathrm{C} \mathrm{NMR}(100 \mathrm{MHz}$, $\left.\mathrm{CDCl}_{3}\right) \delta 165.2,148.5,141.7,140.2,133.3,131.0,130.5,130.3,128.8(2 \mathrm{C}), 128.4(2 \mathrm{C}), 127.9,127.3,123.2,52.2,51.6 .[\alpha]_{\mathrm{D}}{ }^{20}=+1.0(\mathrm{c}=1.12$, $\left.\mathrm{CHCl}_{3}\right)$. 
${ }^{1} \mathrm{H}-\mathrm{NMR}$

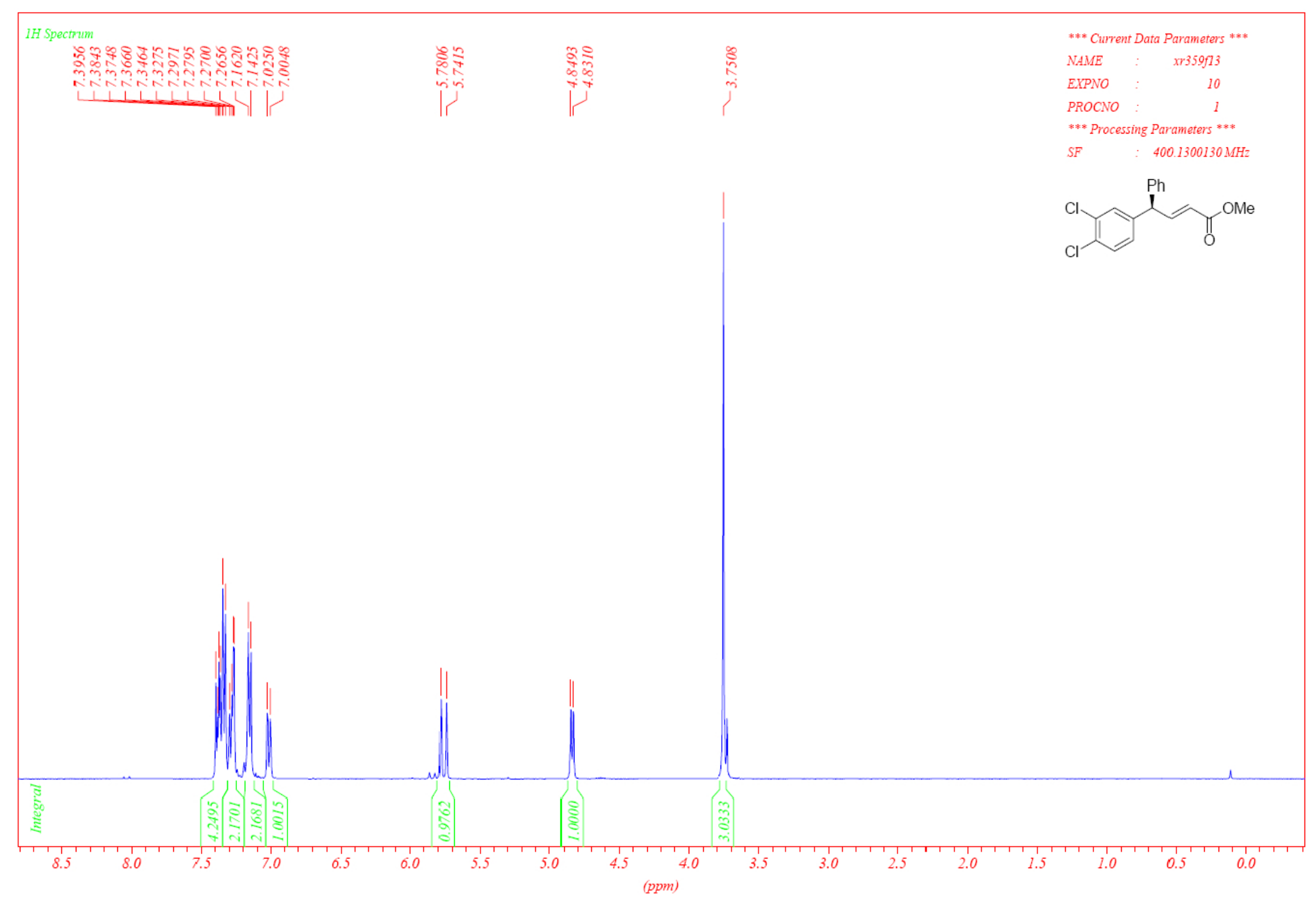




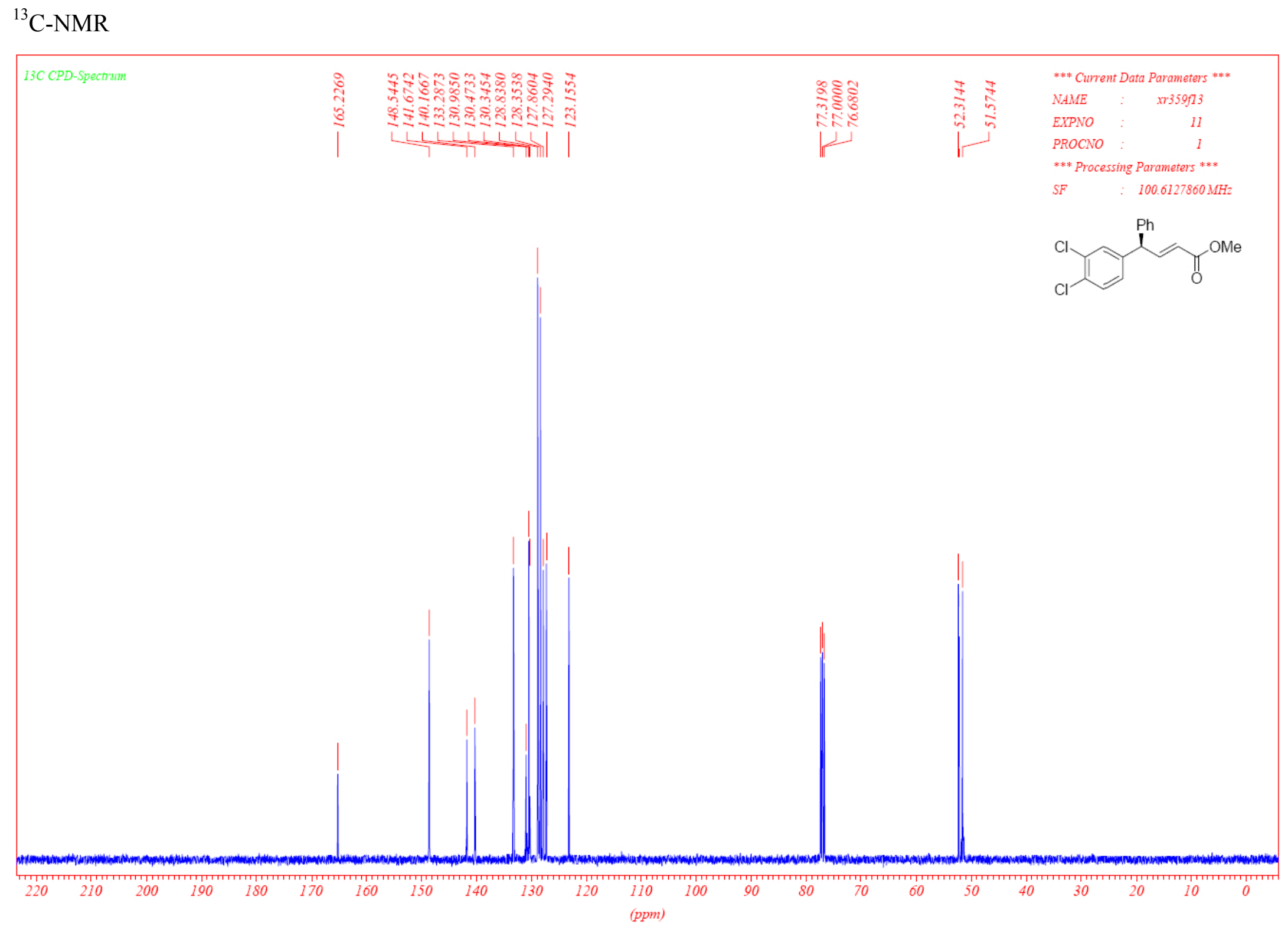




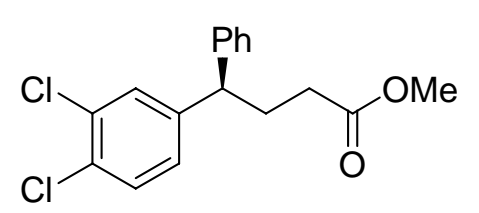

\section{(R)-methyl 4-(3,4-dichlorophenyl)-4-phenylbutanoate 24:}

$\mathrm{Cu}(\mathrm{OAc})_{2} \cdot \mathrm{H}_{2} \mathrm{O}(3.8 \mathrm{mg}, 5 \%)$ and $( \pm)$-BINAP $(12.6 \mathrm{mg}, 5 \%)$ were added into the flask containing $4 \mathrm{ml}$ of THF.

After stirring 5 minutes, PMHS (100 $\mu \mathrm{l}, 1.62 \mathrm{mmol}, 4$ eq.) was added into the flask, following 7 minutes later by compound 23 (130.1 mg, $0.405 \mathrm{mmol}, 1$ eq.) and $t$-BuOH (124.3 mg, $1.62 \mathrm{mmol}, 4$ eq.). The mixture was stirred at RT for 5 hours. The mixture was concentrated in vacuo and the crude product was purified by flash column chromatography (silica, pentane/Et $\mathrm{E}_{2} \mathrm{O} 9 / 1$ ), to afford desired compound as a colourless oil (130.9 mg, 100\% yield). ${ }^{1} \mathrm{H} \mathrm{NMR}\left(400 \mathrm{MHz}, \mathrm{CDCl}_{3}\right) \delta$ 7.36-7.20 (m, 7H), $7.08(\mathrm{dd}, 1 \mathrm{H}, \mathrm{J}=8.4 \mathrm{~Hz}, \mathrm{~J}=1.8 \mathrm{~Hz})$, $3.91(\mathrm{t}, 1 \mathrm{H}, \mathrm{J}=7.3 \mathrm{~Hz}), 3.65$ (s, 3H), 2.35 (m, 2H), 2.27 (m, 2H). ${ }^{13} \mathrm{C}$ NMR $\left(100 \mathrm{MHz}, \mathrm{CDCl}_{3}\right) \delta 173.4,144.5,142.6,132.4,130.3,130.2,129.6$, 128.7, 127.6, 127.1, 126.8, 51.5, 49.5, 32.1, 30.1. $[\alpha]_{\mathrm{D}}^{20}=-6.1\left(\mathrm{c}=1.12, \mathrm{CHCl}_{3}\right)$. 
${ }^{1} \mathrm{H}-\mathrm{NMR}$

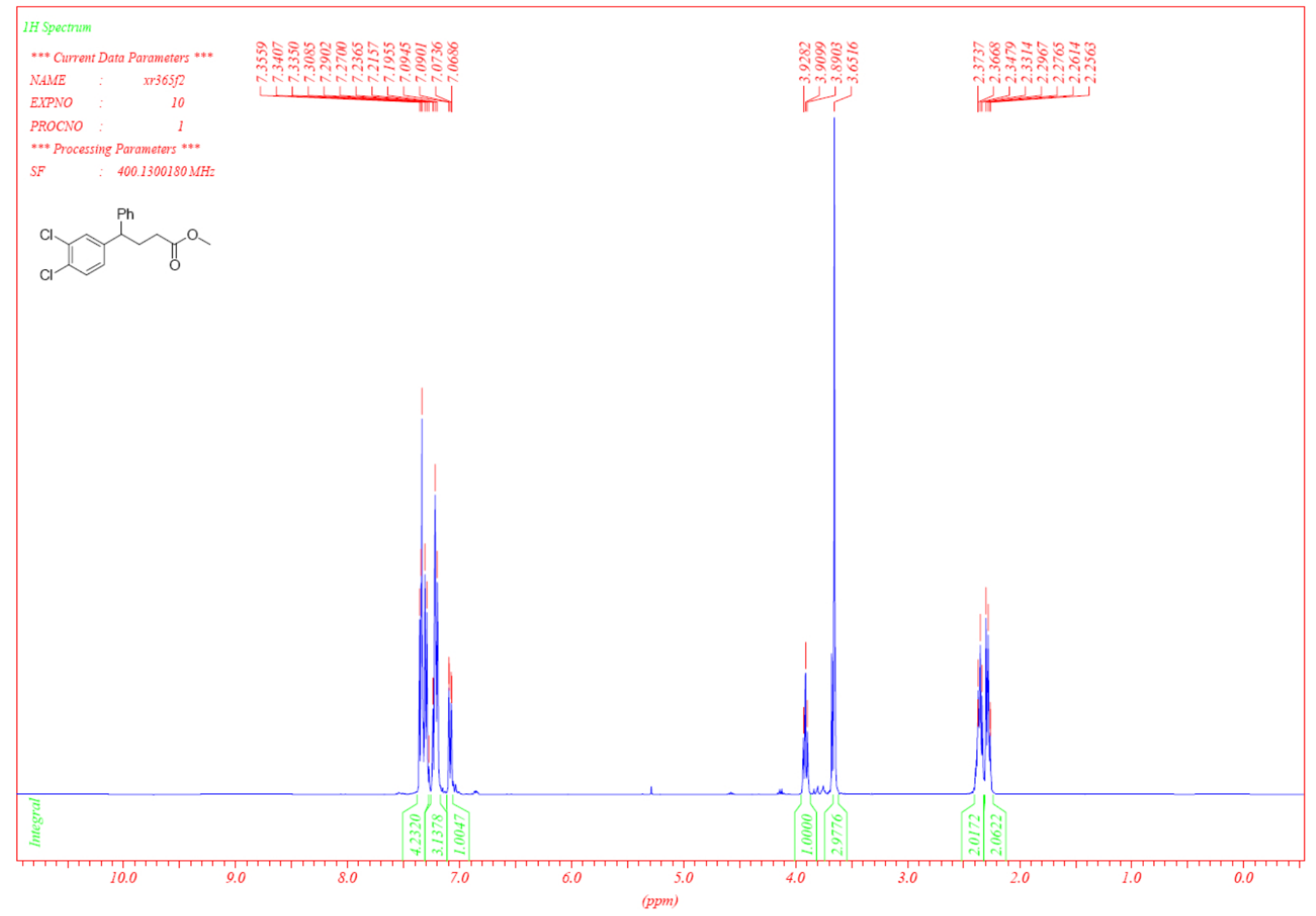




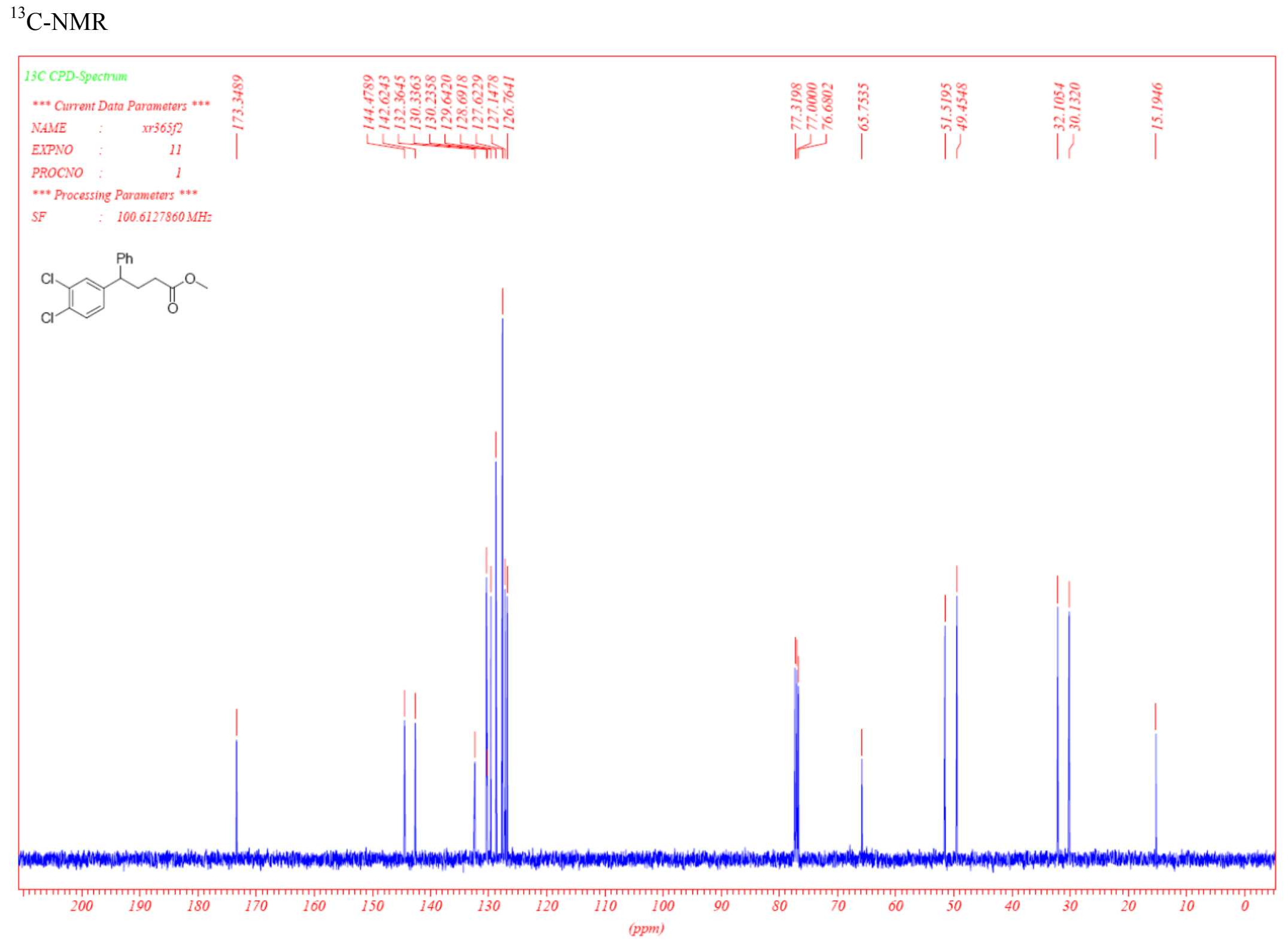

